Systematic Review

\title{
Efficacy of Cannabis-Based Medicines for Pain Management: A Systematic Review and Meta- Analysis of Randomized Controlled Trials
}

J. Aviram, RN, $\mathrm{PhC}^{1}$ and G. Samuelly-Leichtag, PT, $\mathrm{PhC}^{2}$

From: ${ }^{1}$ Faculty of Social Welfare and Health Sciences, University of Haifa, Israel and Rambam Health Care Campus, Haifa, Israel; ${ }^{2}$ The Rappaport Faculty of Medicine, Technion - Israel Institute of Technology, Haifa,

Israel

Address Correspondence: Joshua Aviram, RN, PhC Faculty of Social Welfare and Health Sciences University of Haifa Haifa 31905, Israel

E-mail:shukiaviram@gmail.com

Disclaimer: There was no external funding in the preparation of this manuscript. Conflict of interest: Each author certifies that he or she, or a member of his or her immediate family, has no commercial association (i.e., consultancies, stock ownership, equity interest, patent/licensing arrangements, etc.) that might pose a conflict of interest in connection with the submitted manuscript.

Manuscript received: 09-12-2015 Revised manuscript received:01-18-2016, 02-22-2017 Accepted for publication: 04-12-2017

Free full manuscript: www.painphysicianjournal.com
Background: The management of chronic pain is a complex challenge worldwide. Cannabisbased medicines (CBMs) have proven to be efficient in reducing chronic pain, although the topic remains highly controversial in this field.

Objectives: This study's aim is to conduct a conclusive review and meta-analysis, which incorporates all randomized controlled trials (RCTs) in order to update clinicians' and researchers' knowledge regarding the efficacy and adverse events (AEs) of CBMs for chronic and postoperative pain treatment.

Study Design: A systematic review and meta-analysis.

Methods: An electronic search was conducted using Medline/Pubmed and Google Scholar with the use of Medical Subject Heading (MeSH) terms on all literature published up to July 2015. A follow-up manual search was conducted and included a complete cross-check of the relevant studies. The included studies were RCTs which compared the analgesic effects of CBMs to placebo. Hedges's g scores were calculated for each of the studies. A study quality assessment was performed utilizing the Jadad scale. A meta-analysis was performed utilizing random-effects models and heterogeneity between studies was statistically computed using $\mathrm{I}^{2}$ statistic and tau test.

Results: The results of 43 RCTs (a total of 2,437 patients) were included in this review, of which 24 RCTs (a total of 1,334 patients) were eligible for meta-analysis. This analysis showed limited evidence showing more pain reduction in chronic pain $-0.61(-0.78$ to $-0.43, P<0.0001)$, especially by inhalation $-0.93(-1.51$ to $-0.35, P=0.001)$ compared to placebo. Moreover, even though this review consisted of some RCTs that showed a clinically significant improvement with a decrease of pain scores of 2 points or more, $30 \%$ or $50 \%$ or more, the majority of the studies did not show an effect. Consequently, although the primary analysis showed that the results were favorable to CBMs over placebo, the clinical significance of these findings is uncertain. The most prominent AEs were related to the central nervous and the gastrointestinal (GI) systems.

Limitations: Publication limitation could have been present due to the inclusion of Englishonly published studies. Additionally, the included studies were extremely heterogeneous. Only 7 studies reported on the patients' history of prior consumption of CBMs. Furthermore, since cannabinoids are surrounded by considerable controversy in the media and society, cannabinoids have marked effects, so that inadequate blinding of the placebo could constitute an important source of limitation in these types of studies.

Conclusions: The current systematic review suggests that CBMs might be effective for chronic pain treatment, based on limited evidence, primarily for neuropathic pain (NP) patients. Additionally, GI AEs occurred more frequently when CBMs were administered via oral/oromucosal routes than by inhalation.

Key words: Cannabis, CBMs, chronic pain, postoperative pain, review, meta-analysis

Pain Physician 2017; 20:E755-E796 
T he treatment of chronic pain is based on a combination of pharmacotherapy and complementary non-pharmacotherapy treatment (1-3). The approach for pharmacological treatment for the relief of chronic pain is based primarily on pain intensity. This approach determines that mild pain should be treated with "simple" analgesics, whereas moderate to severe pain should be treated with opioids (4). However, the abundant use of opioids has become a highly controversial topic due to the wide range of problems it presents, such as the large potential for abuse, long-term safety, and difficulty to follow-up on.

\section{Chronology of Cannabis}

Cannabis sativa is thought to have been grown for at least 12,000 years, initially for fiber and grain. The earliest use of cannabis as a medicine is attributed to the Chinese emperor Shen Nung, around $2700 \mathrm{BC}$. The first evidence of the medical use of cannabis came from the fourth century burial in a cave west of Jerusalem; archeologists concluded that cannabis had been burnt in a vessel and was used by smoke inhalation to reduce pain during an unsuccessful child delivery (5). The Irish physician William Brooke O'Shaughnessy (6) first introduced the analgesic effect of cannabis to the Western world in a pioneer study in 1839. Today, the pain management field is largely leaning towards the research of cannabis-based medicines (CBMs) which have become some of the most debated medicines todate. The paradigmatic change that accelerated the use of cannabinoids for pain treatment started in the 1960s. In 1964, Mechoulam and Gaoni (7) identified tetrahydrocannabinol (delta-9-THC) as the main psychoactive component of cannabis. Additionally, Lester Grinspoon (8) described the positive effect of cannabinoids for the treatment of intractable conditions. From a clinical standpoint, most physicians grasp CBMs as a palliative treatment or as a part of a multi-model pain treatment. On the other hand, the use of CBMs is poorly taught in the training of physicians due to the scarcity of quality randomized controlled trials (RCTs) in this field (9).

\section{Cannabis Research Agendas}

In pain clinics across Canada, the rate of the use of CBMs is estimated to be between 12-15\% (10). Unlike opioids, there is a minority of cannaboid (CB) receptors in the brain stem in the areas that control breathing, which explains the low toxicity of cannabinoids (11). This is one of the main reasons that further research is needed on the use of CBMs for pain treatment. However, clinical research on CBMs is limited in quality and quantity (mainly when utilized by smoking and inhalation), due to the lack of research funding in this field. Furthermore, studying the positive effects of CBMs is in contrast to the global anti-smoking and anti-drugs strategies, and until those issues can be resolved, no large-scale phase III study can be made on the efficacy of herbal cannabinoids. In terms of drug trials, an overwhelming leap has been made from phase II trials directly to phase IV trials for the use of this complex botanical compound (9).

Clinically, the most common route of administration for CBMs is by inhalation, or smoking (12), followed only by ingestion. Other routes of administration include rectal, sublingual, transdermal, ocular, and intravenous (13). Despite the significant discoveries that support the therapeutic potential of CBMs, the health hazards of smoking (14) combined with the cognitive and emotional impacts that are related to cannabinoid use have generated regulatory obstacles worldwide (15). However, the use of cannabinoids as a medication is becoming more common (16), and there is ample development in the routes of administration. Specifically, new technologies are being implemented in order to simplify the medicinal use of cannabinoids, decrease side effects, and provide a constant level of cannabinoid in the blood stream (17).

\section{Cannabinoids: Clinical Trials, Reviews, and Meta-Analyses Chronology}

Thus far, 9 reviews of RCTs have been published regarding the effects of $\mathrm{CBMs}$ on pain. The earliest of them was published in 2001, by Campbell et al (18), which summarized studies that used single-dose CBM for the treatment of various types of chronic malignant and non-malignant pain in comparison to codeine or to codeine and placebo. The authors reported that, in general, no benefit was found for CBMs over codeine, but over placebo only. Two additional reviews reported conflicting results regarding the efficacy of CBM use for the treatment of various types of pain conditions including postoperative, visceral, cancer, and neuropathic pain (NP) $(19,20)$. The effects of CBMs (e.g., nabilone, dronabinol, and THC analogue) and smoked cannabinoids were examined in several recent RCT studies; the appraising of those studies in 3 different reviews revealed overall evidence that cannabinoids are safe and moderately effective for the investigated diagnoses (21-23). Furthermore, the 3 most recent reviews of RCTs 
showed that CBMs may provide effective analgesia for non-malignant NP (24) and for chronic non-malignant pain (25). One last review of 6 trials that included 325 chronic pain patients and 6 trials that included 396 NP patients suggested that cannabinoids may be efficacious for NP (26). Additionally, many more reviews were published regarding the effect of CBMs on pain, however, these studies were limited by case-based, anecdotalbased, or laboratory-based scientific research on headaches (27), NP (28-31), chemotherapy-induced pain (32), multiple sclerosis (MS) pain (33), HIV-associated sensory neuropathy (34), and rheumatoid arthritis pain (35). Finally, a few conference proceedings also investigated the effects of CBMs on pain (36), more specifically on musculoskeletal pain (37) and NP (38).

To this date, 4 meta-analyses summarized the efficacy and safety data of CBMs for chronic pain versus placebo; each has specific merits and faults (39-42). The first meta-analysis, by Iskedjian et al (39), investigated 7 (9 publications) randomized, double-blinded, placebocontrolled trials involving the use of CBMs in the treatment of pain associated with MS or other types of NP (43-51). All of these trials showed a significant decrease in pain intensity (ranging from 1.5-1.7 improvement, on a scale of $0-10$ ) by CBMs, but also reported a significant decrease in pain intensity (a 0.8 improvement) by placebo. The authors explained this data by noting that 2 of the studies allowed patients to freely use rescue medications $(48,50)$. Removing these studies lowered the placebo effect to 0.6-point improvement, which caused the effect to render non-significant. The second meta-analysis, by Martín-Sánchez et al (40), reviewed 18 double-blind RCTs having a crossover or parallel design $(43,45-48,52-61)$ and included 7 of them in the meta-analysis $(45,48,56,57,60,61)$ [one of the reviewed studies included 2 phases (60)], comparing any type of cannabinoid preparation to placebo in chronic pain patients. This meta-analysis presented an overall effect size of -0.61 , favoring CBMs over placebo for pain reduction. The third meta-analysis, by Whiting et al (41), meta-analyzed many medicinal effects of CBMs including nausea and vomiting response, pain reduction, and spasticity reduction, as well as the assessment of AEs. Overall, this meta-analysis included in their primary analysis 8 double-blind RCTs having a parallel design (62-68), along with one that was not published (69) and showed a non-significant, higher incidence of pain reduction due to CBMs ( $n=254$, total $=685$ ) compared to placebo $(n=215$, total $=685$ ). Further analyses showed a pain reduction effect size of $-0.46(95 \% \mathrm{Cl},-0.80$ to
-0.11). Nonetheless, this study was later claimed to have some methodological weaknesses in the letter to the editor section of the same journal; one claim was regarding the lack of integration of some of the studies in the primary analysis and another claim which consisted of 2 issues: the lack of separation between chronic pain conditions and the shortage in the AEs' complete description (70). The fourth and most recent meta-analysis is by Andreae et al (42), meta-analyzed 5 RCTs $(62,71-74)$, focusing only on the effect of inhalation of cannabinoids for chronic NP. This meta-analysis consisting of 178 patients with follow-up ranging from days to weeks, showed short term pain intensity reduction by $30 \%(75,76)$, with numbers needed to treat (NNT) (42) 5.6 for cannabis comparable to NNT of 5.9 for gabapentin (77). Their findings suggest that inhaled cannabinoids could potentially rival currently available therapeutics for chronic NP (78), whose NNT is comparable (42), but typically range above 8 (7981). However, Deshpande et al (82) later concluded that the studies that Andreae et al (42) used in their analysis had challenges with masking. Data could not be pooled owing to heterogeneity in THC potency by dried weight, differing the frequency and duration of treatment, and variability in assessing the outcomes. Consequently, the clinical relevance of the findings from any of the 4 meta-analyses is unknown (39-42).

Taken together, the chronological changes in the aforementioned results show an interesting trend in the beneficial effect of cannabinoids on pain. These findings can be carefully explained by the pharmacological advancement in this field, but may also be due to society's favorable attitude toward the use of CBMs for pain treatment purposes. Furthermore, many of these studies included only a single selected dose and did not investigate the most commonly used route of CBMs, i.e., inhalation. Another explanation for the discrepancy in the reviews can be due to inter-individual variation in the analgesic response to CBMs, which is associated with the different routes of administration, bioavailability, or use of additional medications that can interact with CBMs.

\section{Study Rationale}

The conflicting results of the existing reviews and meta-analyses leave the question regarding the efficacy of CBMs in the treatment of postoperative and chronic pain unanswered. Moreover, the existing metaanalyses did not include all of the studies available at this time (5-8 trials), instead of the available 24 studies. 


\section{Study Objectives}

Consequently, there is a need to conduct a new, conclusive review that will present all the specific information of the compatible trials, as well as to conduct meta-analyses, which will incorporate all of the comparable RCTs in order to update the knowledge of clinicians and researchers, regarding the efficacy and the entire range of expected adverse events (AEs) of CBMs for pain treatment.

\section{Methods}

\section{Information Sources}

The study was conducted according to the PRISMA statement (83). An electronic search was made in Medline/Pubmed and in Google Scholar by the use of Medical Subject Heading (MeSH) terms on all literature published until July 2015.

\section{Search Strategy}

We used the following search terms by the use of the Boolean combination of: "cannabis" OR "cannabinoids" OR "marijuana" OR "THC" OR "tetrahydrocannabinol" AND "pain", "chronic pain" AND "postoperative pain". This was followed by a manual search in all of the reviews regarding this topic. The search covered only full text manuscripts, published in English language.

\section{Eligibility Criteria and Study Selection}

The selected studies were double-blind, RCTs (most placebo-controlled, some included active drugs and placebo comparisons, and few included only active drugs comparisons) with a crossover or parallel design. In the intervention group, patients were to have received any type of cannabis preparation, by any route of administration. Synthetic derivates of THC, such as dronabinol, nabilone, sativex/nabiximol, cannabidiol, CT-3, ajulemic acid, synthetic nitrogen analog of tetrahydrocannabinol (NIB), cannabinoid cigarettes/vaporizer, cannabinoid extract, fatty acid amide hydrolase-1 (FAAH1) inhibitor (PF-04457845), levonantradol, and benzopyranoperidine (BPP), were included. In the control group, patients were to have received either placebo, whether "identical" or not, or an "active" (weak opioids or naproxen) treatment. The patients included were to have been suffering from either pre-existing chronic pain or postoperative pain. RCTs including healthy volunteers were excluded.

\section{Data Collection Process and Quality Assessment}

The 2 authors (Aviram and Samuelly) of this review assessed the studies' quality independently, and there were no disagreements in scoring that needed to be resolved. Even though the studies were not required to have a quality threshold level other than the preexisting inclusion criteria, their validity was assessed by the Jadad scale (84). The majority of the included trials showed moderate to high quality of evidence (Tables 1-3). To conclude our findings, according to the Jadad scale (84), 10 studies received a " 5 " score, showing high quality evidence. Thirty-one studies received a "3-4" score, showing moderate quality evidence. Only 2 studies received a "1-2" score, showing low quality evidence (the assessment of each study and the total scores with component item scoring are provided in Table 4.

\section{Outcome Measures (Data Items)}

The efficacy measure that was chosen was the variable "pain intensity", as scored by the numerical rating scale (NRS-11) (85), numerical 11 point box (BS-11) (86), visual analog scale (VAS) (87), and the VAS section of the questionnaire short form McGill Pain Questionnaire (88). In all of the studies, the range of pain intensity was required to be from $0-10$; the studies that used scales ranging from 0-100 were converted in order to evaluate all of the studies on the same range.

AEs were assessed by analyzing the number of AEs experienced in each sample (incidence). The AEs were categorized by the effected systems (Tables $5-7$ ).

\section{Risk of Limitations in Individual Items}

Of the 24 studies included in the meta-analysis, 12 relied on 2 intervention arms/groups (CBMs vs. placebo) $(45,46,48,59,61,64-66,89-92)$. While other studies used a placebo control group and various intervention arms, using diverse cannabinoid preparations $(43,47,56,57,60,63,93,94)$ or increasing doses of the same preparation over long periods of time $(45,47,48,59,90-92)$.

Furthermore, unlike former meta-analyses that evaluated the influence of CBMs on chronic pain (39$41)$, this meta-analysis included data from studies that examined the effects of CBMs on postoperative pain $(89,93)$; these studies were included in the analysis for the examination of the influence of the overall effect size of CBMs on pain (Fig. 1). Consequently, they were redacted in the following analyses because of the weak 
Effective of Cannabis-Based Medicines for Pain Management

\begin{tabular}{|c|c|c|c|c|c|}
\hline 憘 & + & $H$ & a & n & $m$ \\
\hline : & 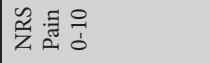 & 荎官 & 过市 & 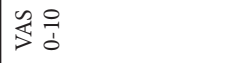 & 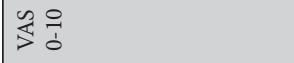 \\
\hline 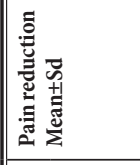 & 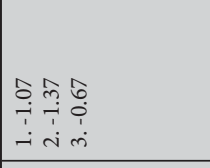 & 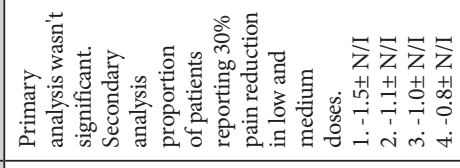 & 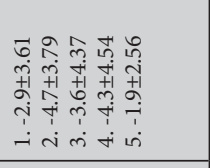 & 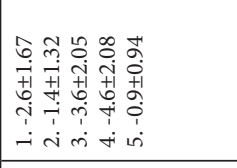 & 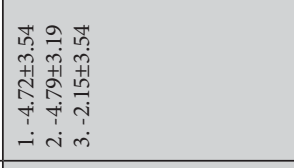 \\
\hline$\stackrel{3}{3}$ & 5 & $\overline{\mathrm{z}}$ & $\stackrel{z}{t}$ & $\exists$ & 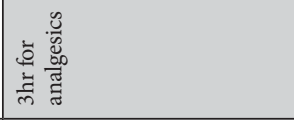 \\
\hline 鴶苞 & 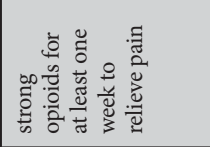 & 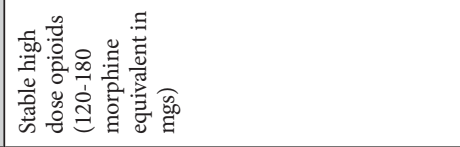 & $\overline{\mathbf{z}}$ & 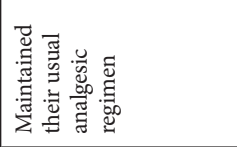 & 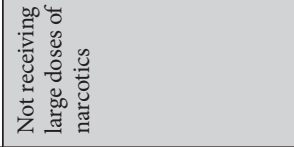 \\
\hline 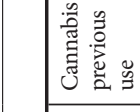 & $\bar{z}$ & 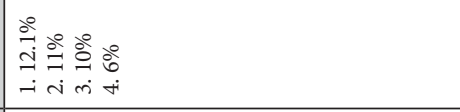 & $\overline{\mathrm{z}}$ & $\bar{z}$ & $\overrightarrow{\mathrm{z}}$ \\
\hline 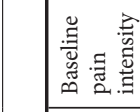 & 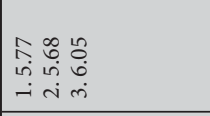 & 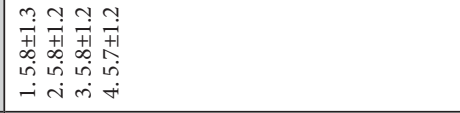 & $\bar{z}$ & $\overrightarrow{\mathrm{z}}$ & $\overline{\mathrm{z}}$ \\
\hline 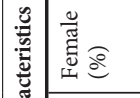 & 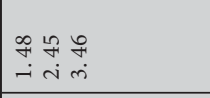 & 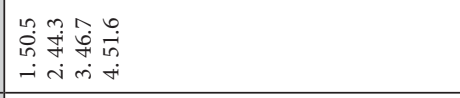 & $\bar{z}$ & $\bar{z}$ & $\bar{z}$ \\
\hline 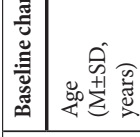 & 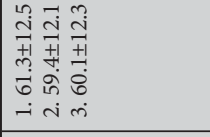 & 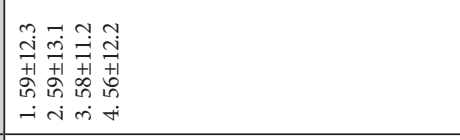 & \begin{tabular}{|l} 
\\
$\bar{z}$ \\
+1 \\
\end{tabular} & 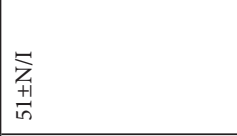 & $\begin{array}{l}n \\
\stackrel{n}{N} \\
\end{array}$ \\
\hline 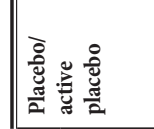 & 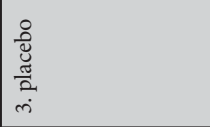 & 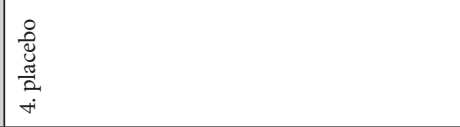 & 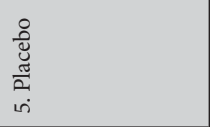 & $\begin{array}{l}\text { 浐 } \\
\frac{\pi}{2} \\
\text { in } \\
\text { in }\end{array}$ & 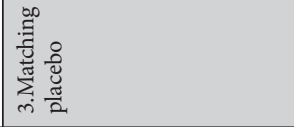 \\
\hline 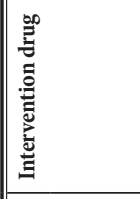 & 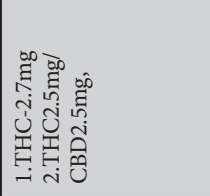 & 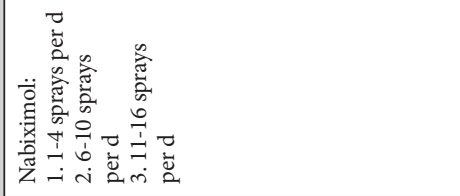 & 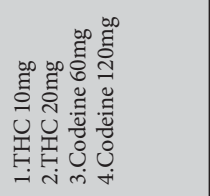 & 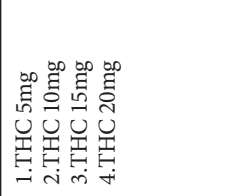 & 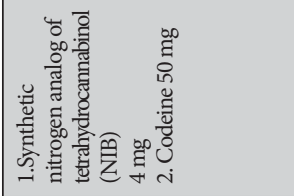 \\
\hline 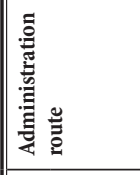 & 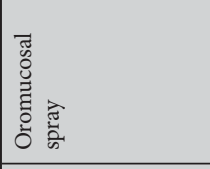 & 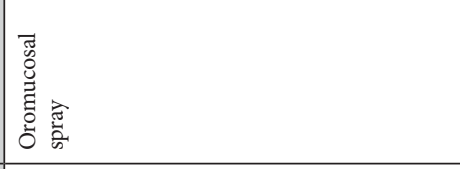 & 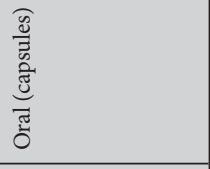 & 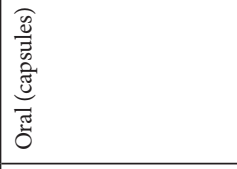 & 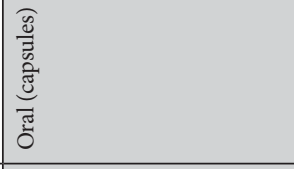 \\
\hline 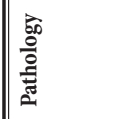 & 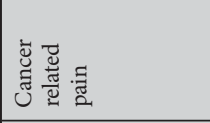 & 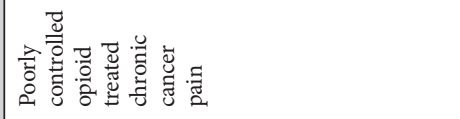 & 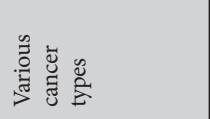 & 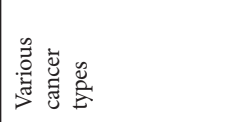 & 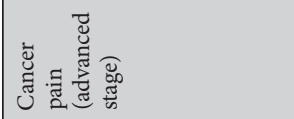 \\
\hline $\mathrm{z}$ & 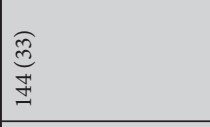 & 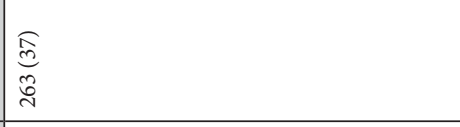 & 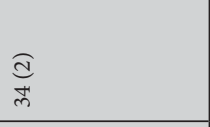 & 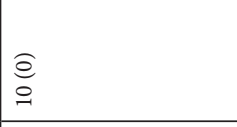 & 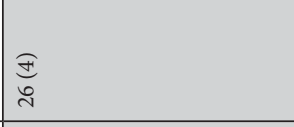 \\
\hline 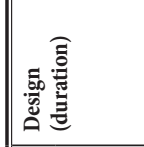 & 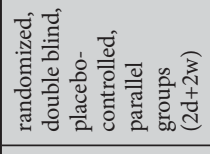 & 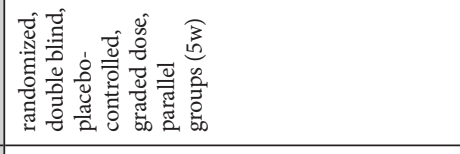 & 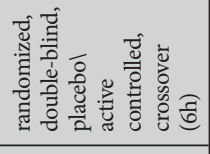 & 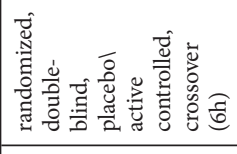 & 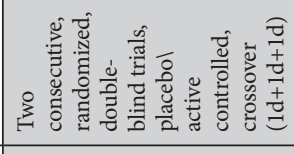 \\
\hline 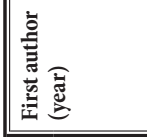 & 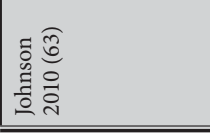 & 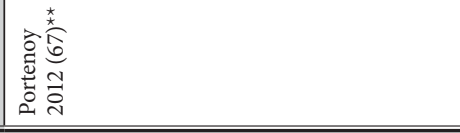 & 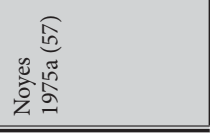 & 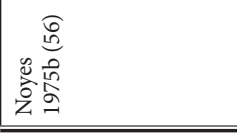 & 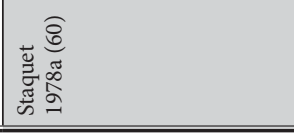 \\
\hline
\end{tabular}


Pain Physician: September/October 2017: 20:E755-E796

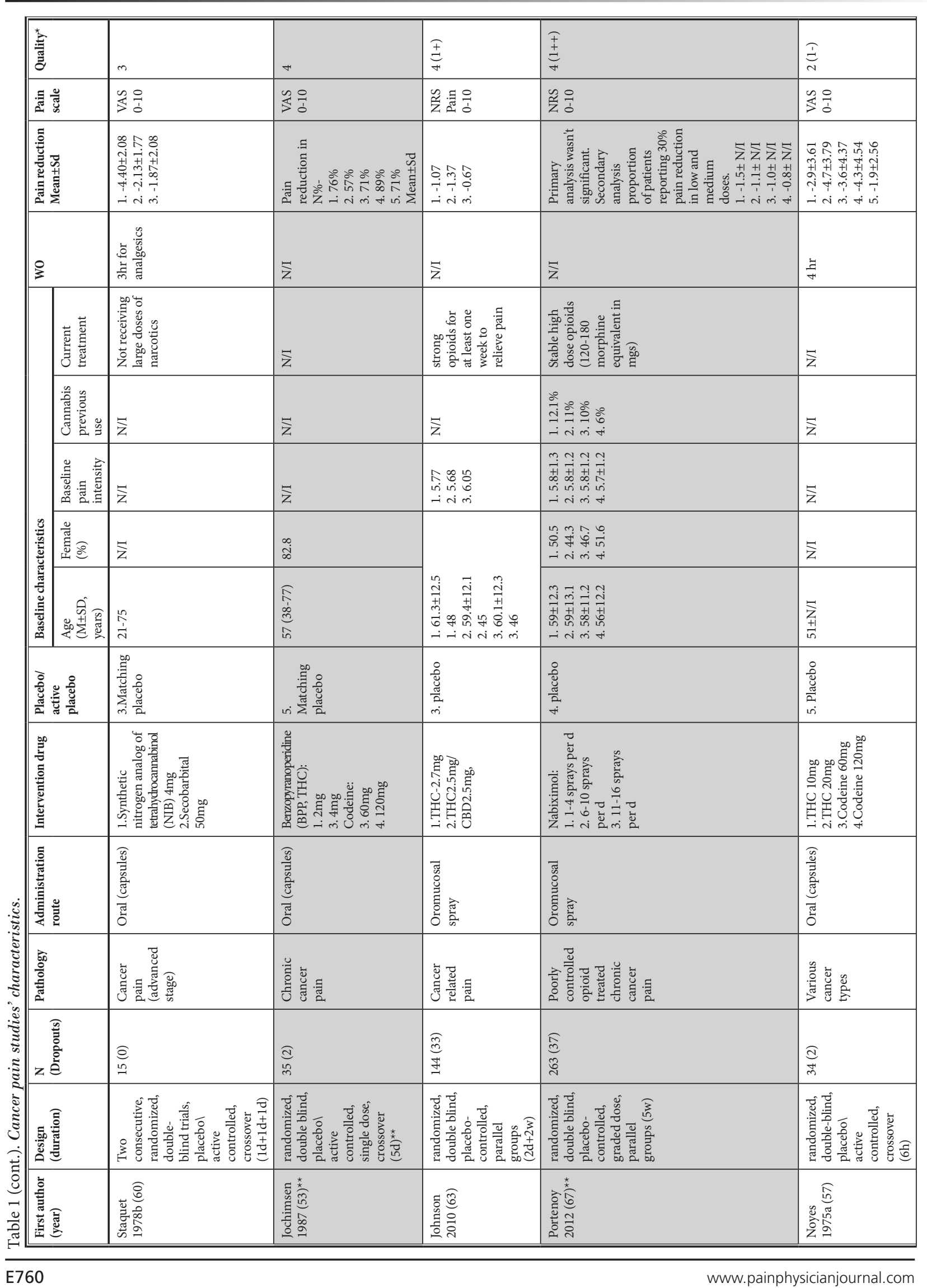


Effective of Cannabis-Based Medicines for Pain Management

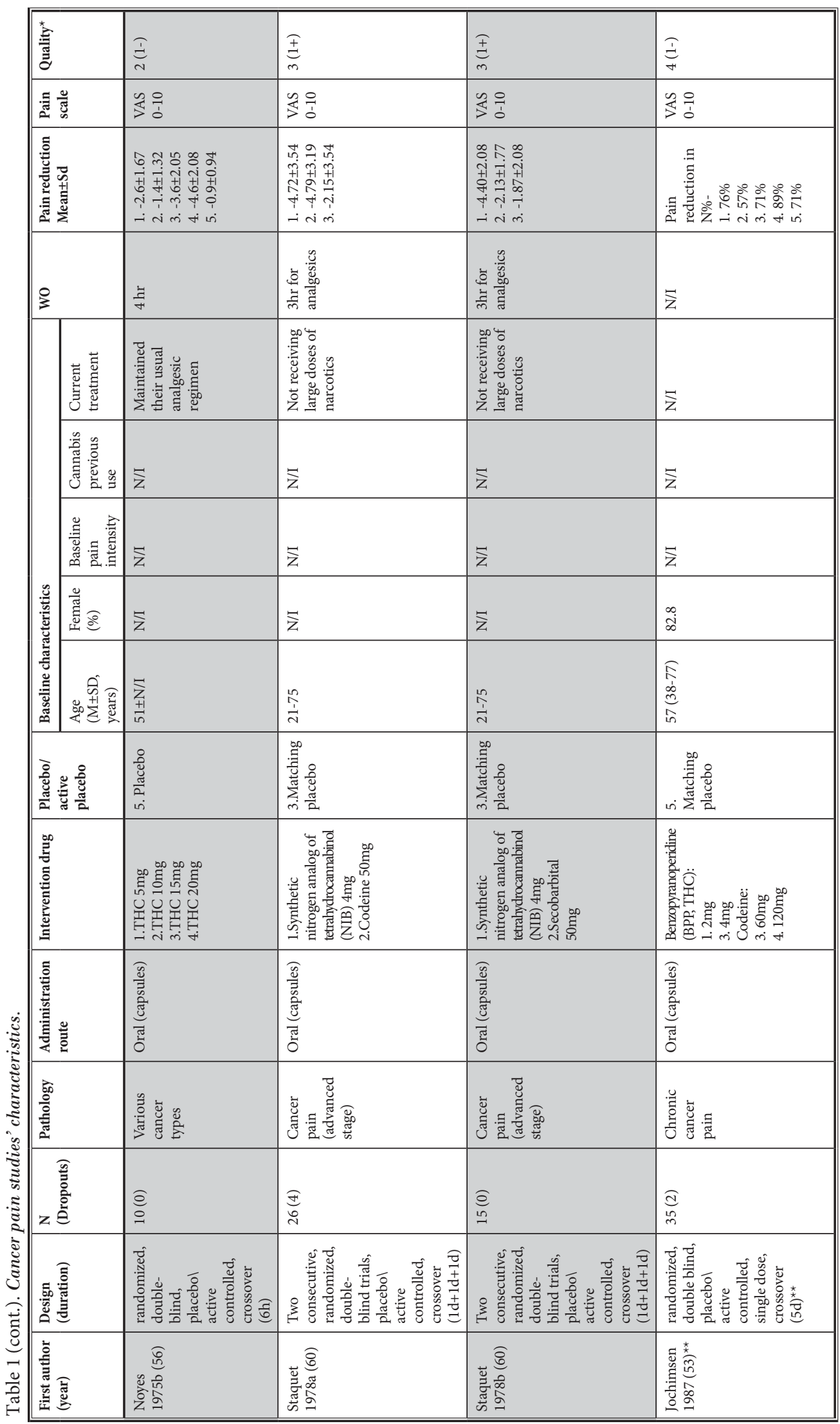

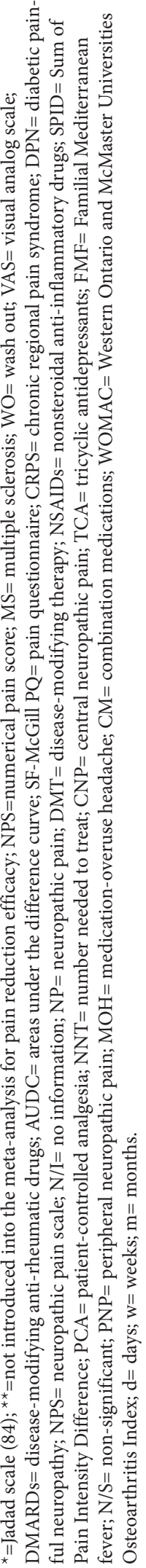


Pain Physician: September/October 2017: 20:E755-E796

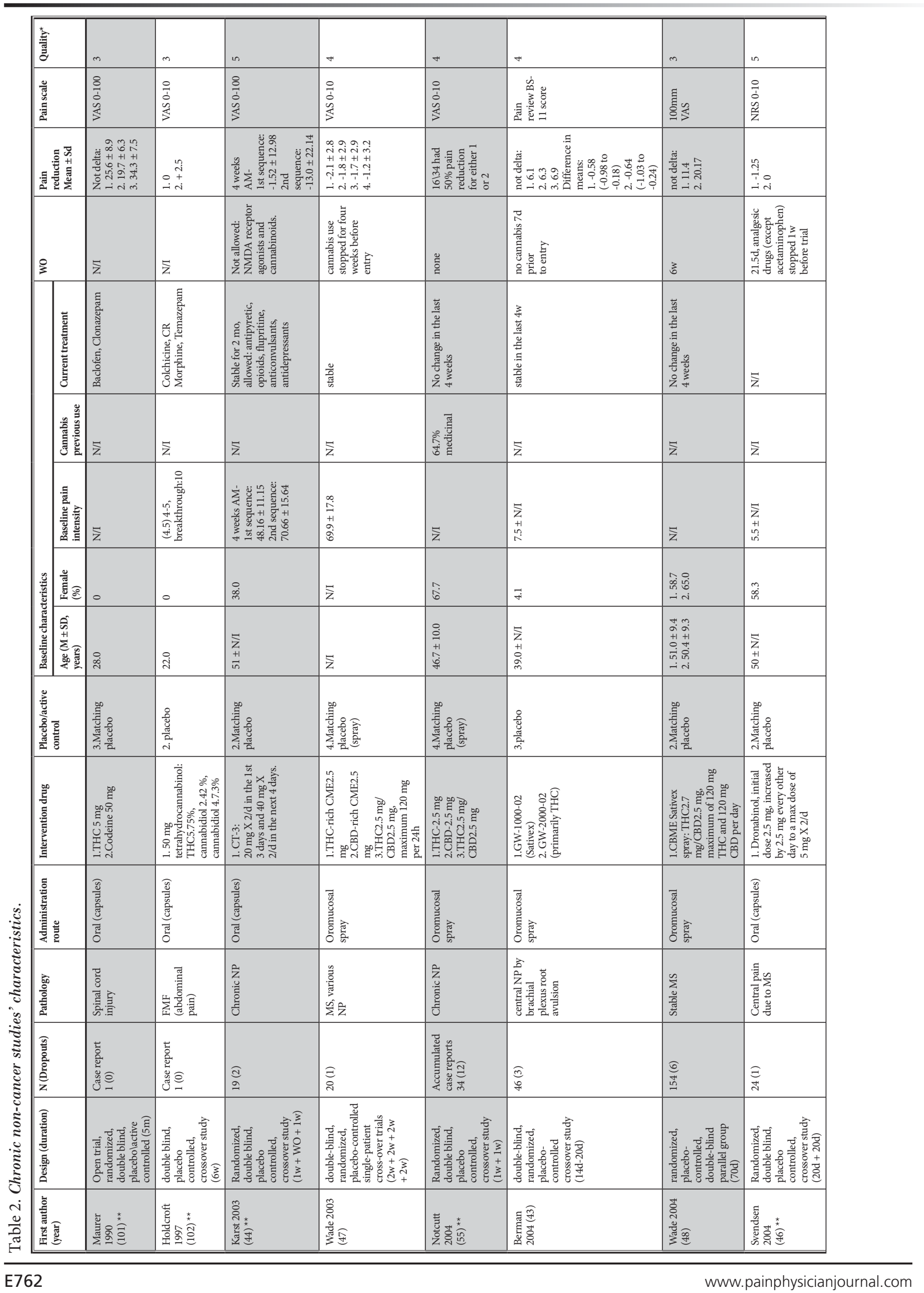


Effective of Cannabis-Based Medicines for Pain Management

\begin{tabular}{|c|c|c|c|c|c|c|c|c|c|c|}
\hline & 喜 & in & H & $m$ & m & in & in & in & H & + \\
\hline & 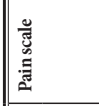 & 丵言 & 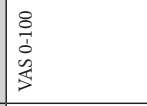 & 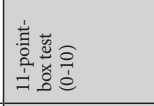 & 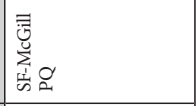 & \begin{tabular}{|l}
8 \\
$\frac{1}{1}$ \\
$\vdots$ \\
5 \\
5 \\
\end{tabular} & 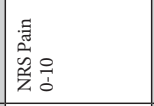 & $\begin{array}{l}8 \\
\frac{1}{0} \\
0 \\
\frac{5}{1} \\
1\end{array}$ & 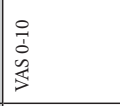 & $\begin{array}{l}0 \\
\vdots \\
\vdots \\
\vdots \\
>\end{array}$ \\
\hline & 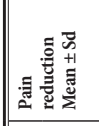 & 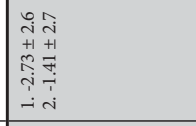 & 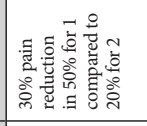 & 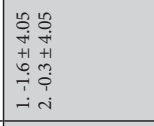 & 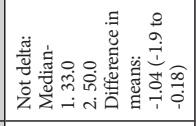 & 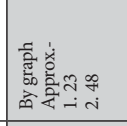 & 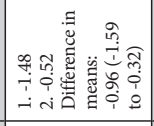 & 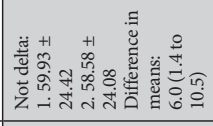 & 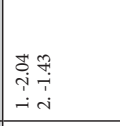 & 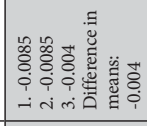 \\
\hline & 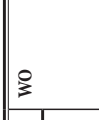 & 竒 & $\pi$ & 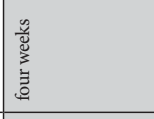 & $\bar{\Sigma}$ & 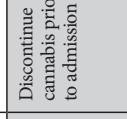 & $\bar{\Sigma}$ & 产 & $\bar{z}$ & 部 \\
\hline & 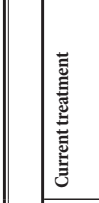 & 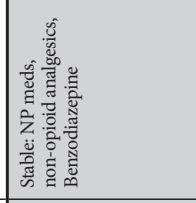 & 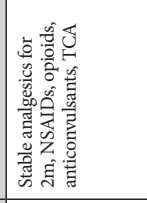 & 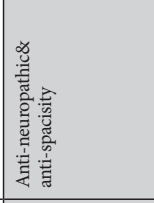 & 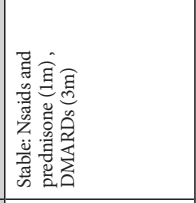 & 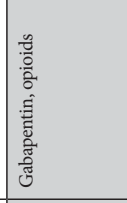 & 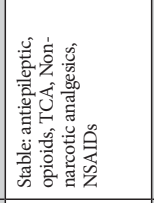 & 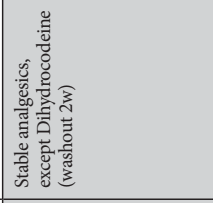 & 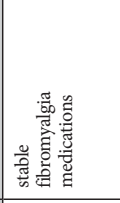 & 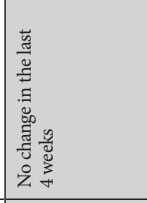 \\
\hline & 童 & 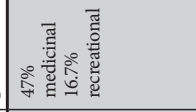 & 旁 & 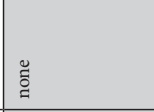 & 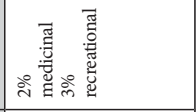 & 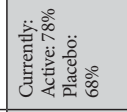 & 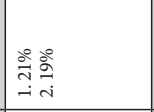 & $\bar{z}$ & 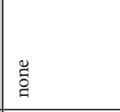 & $\bar{z}$ \\
\hline & 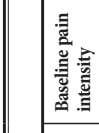 & 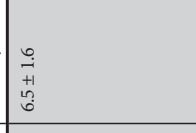 & $\bar{\Sigma}$ & 8 & 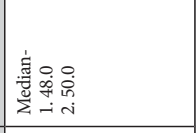 & 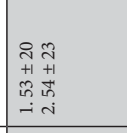 & 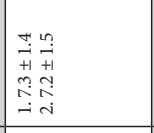 & 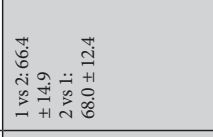 & 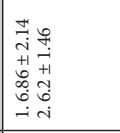 & $\mid$\begin{tabular}{|c}
$\vec{n}$ \\
0 \\
0 \\
0
\end{tabular} \\
\hline & 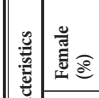 & 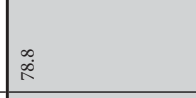 & $\infty$ & zี & $i$ & : & 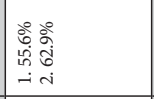 & 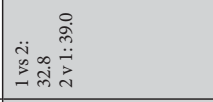 & $\bar{z}$ & $\frac{3}{7}$ \\
\hline & 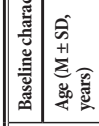 & 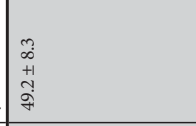 & 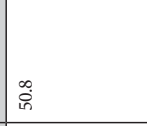 & 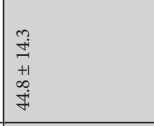 & 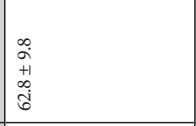 & 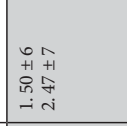 & 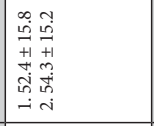 & 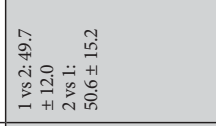 & 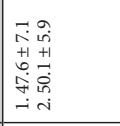 & 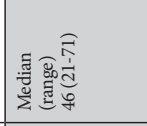 \\
\hline & 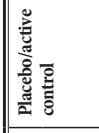 & 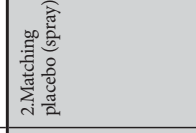 & 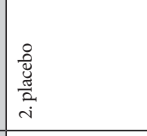 & 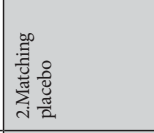 & 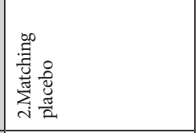 & $\mid$ & 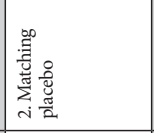 & 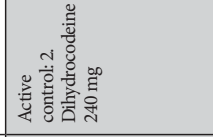 & 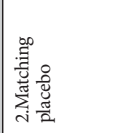 & 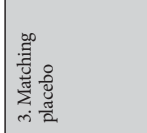 \\
\hline & 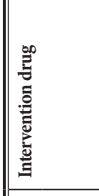 & 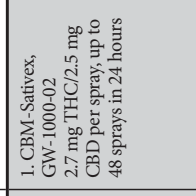 & 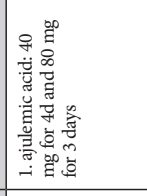 & 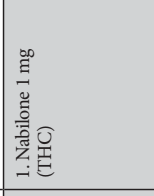 & 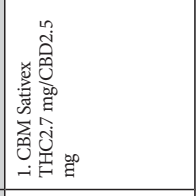 & 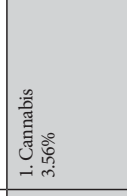 & 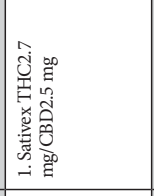 & 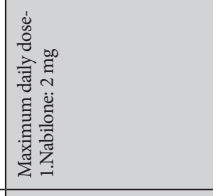 & 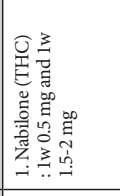 & 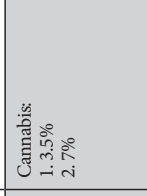 \\
\hline & 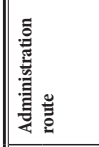 & 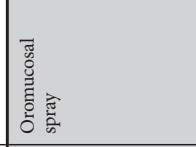 & 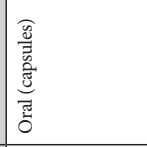 & 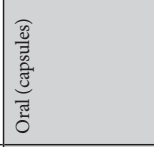 & 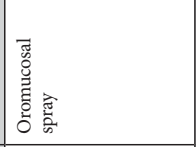 & 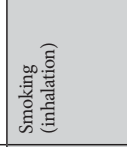 & 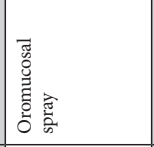 & 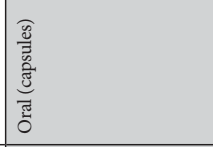 & 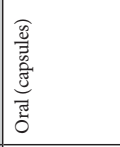 & 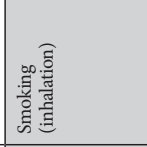 \\
\hline & 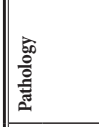 & 产: & $\hat{\mathrm{z}}$ & 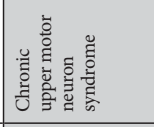 & 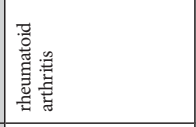 & 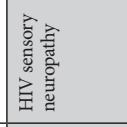 & 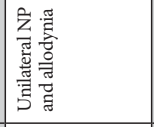 & 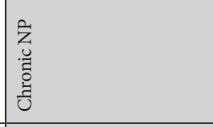 & 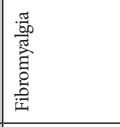 & 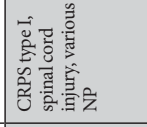 \\
\hline & 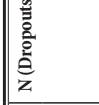 & \begin{tabular}{|l}
$\widehat{a}$ \\
0 \\
0
\end{tabular} & ฮ & $\frac{\Omega}{\Xi}$ & 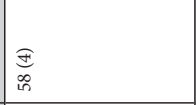 & \begin{tabular}{|l}
$\frac{\pi}{2}$ \\
$\vdots$ \\
0
\end{tabular} & 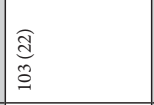 & 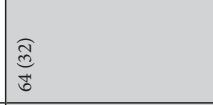 & \begin{tabular}{|l} 
\\
$G$ \\
$G$
\end{tabular} & \begin{tabular}{|l} 
\\
0 \\
$\infty$ \\
$\infty$ \\
\end{tabular} \\
\hline & 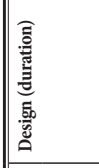 & 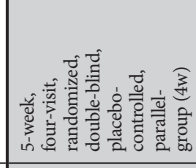 & 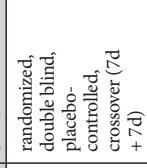 & 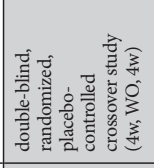 & 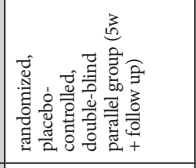 & 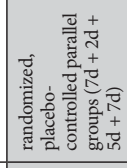 & 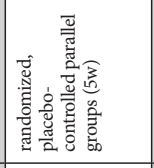 & 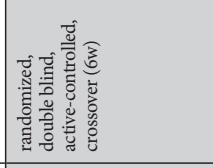 & 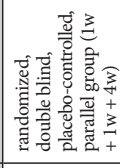 & 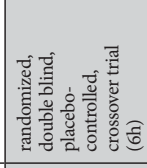 \\
\hline & 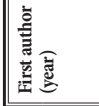 & 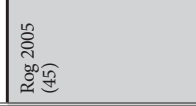 & 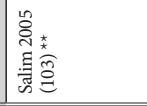 & 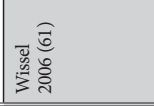 & 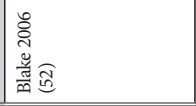 & 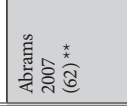 & 竞㔯 & $\begin{array}{l}\text { 总 } \\
\text { 䁘 }\end{array}$ & 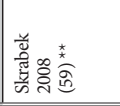 & 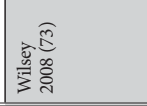 \\
\hline
\end{tabular}


Pain Physician: September/October 2017: 20:E755-E796

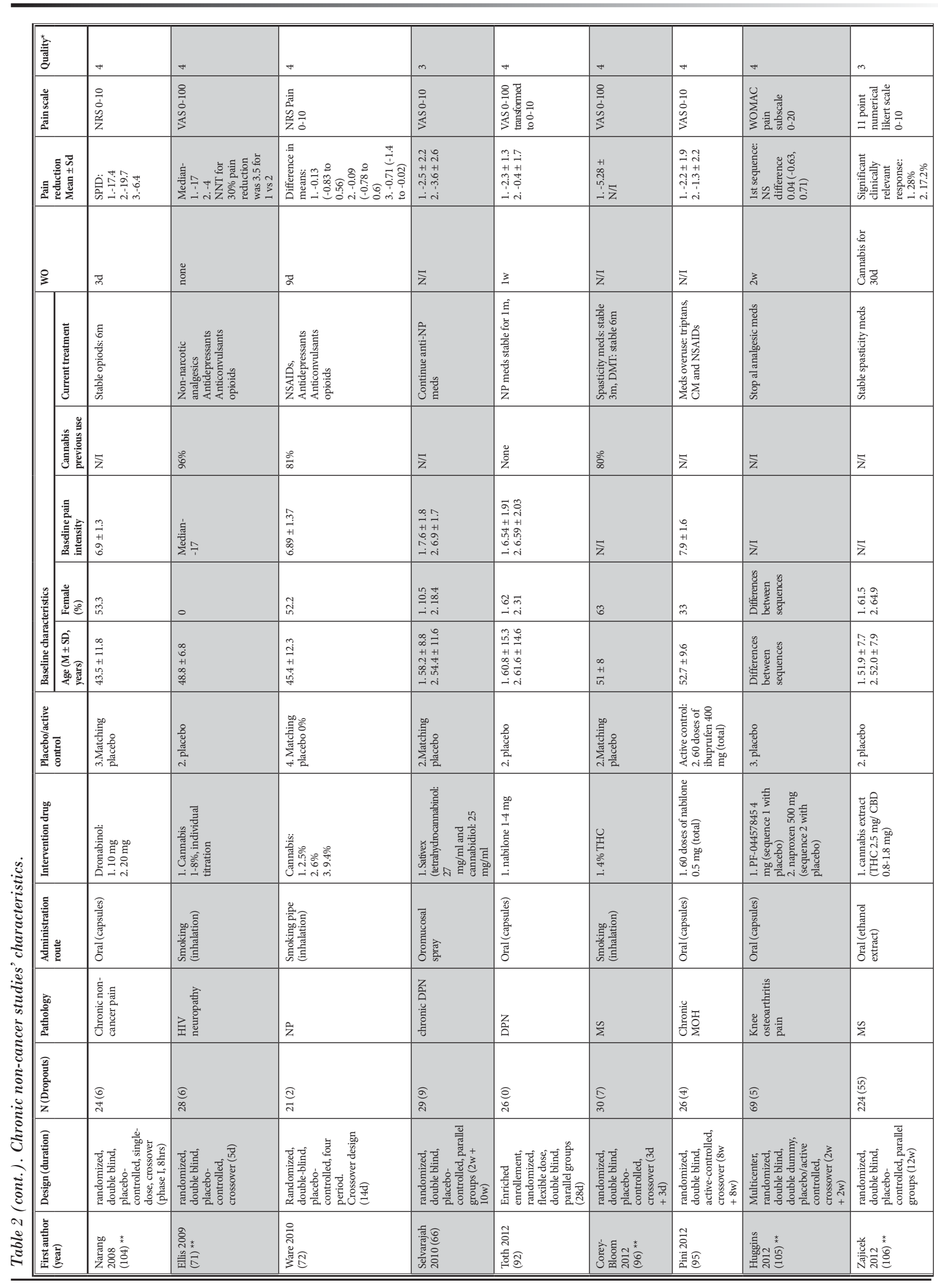


Effective of Cannabis-Based Medicines for Pain Management

\begin{tabular}{|c|c|c|c|c|c|c|c|c|}
\hline 衰 & + & in & in & in & m & in & + & " \\
\hline 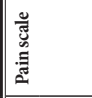 & 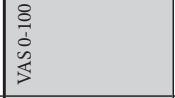 & 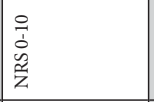 & 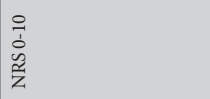 & & 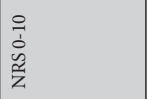 & 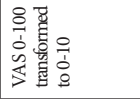 & 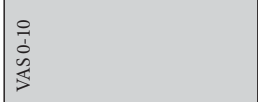 & 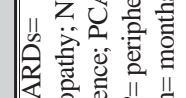 \\
\hline 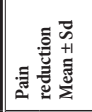 & 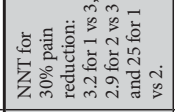 & 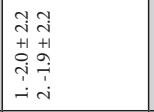 & 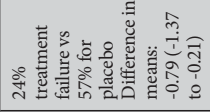 & 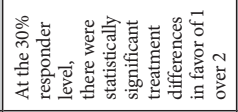 & 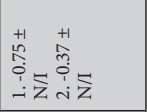 & 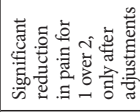 & 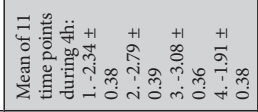 & 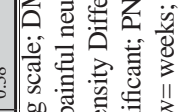 \\
\hline 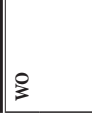 & $\frac{7}{\frac{7}{4}}$ & $\bar{z}$ & $\bar{z}$ & 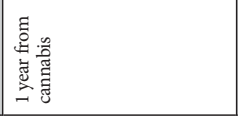 & $\sum_{1}^{2}$ & $\bar{z}$ & $\frac{1}{3}$ & 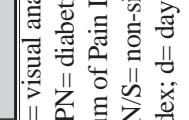 \\
\hline 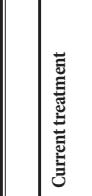 & 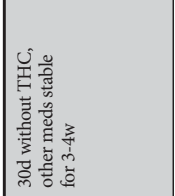 & 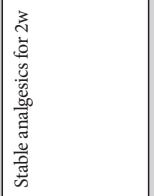 & 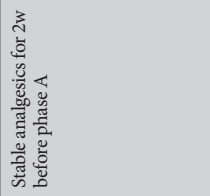 & & 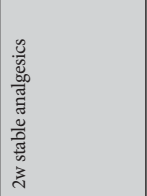 & 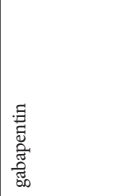 & 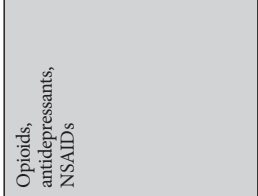 & 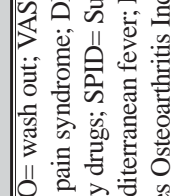 \\
\hline 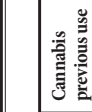 & 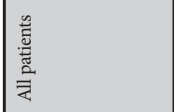 & $\bar{z}$ & 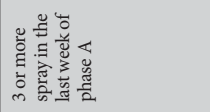 & 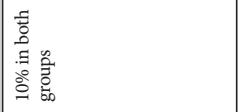 & $\bar{z}$ & $\bar{z}$ & $\bar{\Sigma}$ & 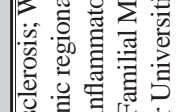 \\
\hline 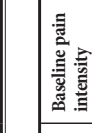 & 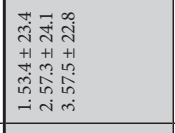 & 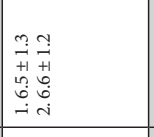 & 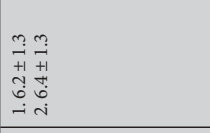 & $\bar{z}$ & 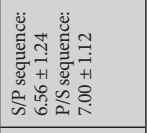 & 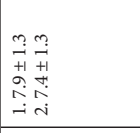 & 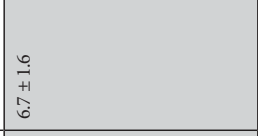 & 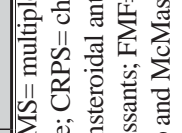 \\
\hline 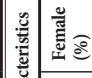 & $\underset{\sim}{\infty}$ & $\begin{array}{l}\infty \\
\substack{\infty \\
-i \\
-i} \\
\end{array}$ & 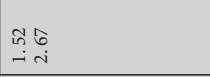 & 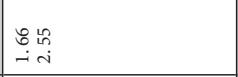 & 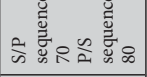 & 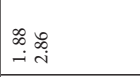 & $\mp$ & 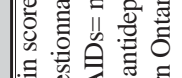 \\
\hline 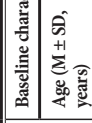 & $\begin{array}{l}= \\
= \\
+1 \\
0 \\
i n\end{array}$ & 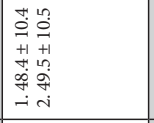 & 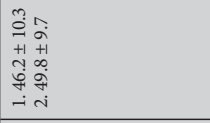 & 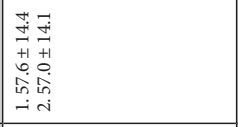 & 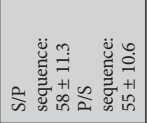 & 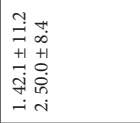 & \begin{tabular}{|l}
$\infty$ \\
$\infty$ \\
+1 \\
0 \\
0 \\
0
\end{tabular} & 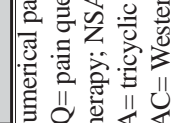 \\
\hline 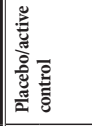 & 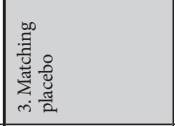 & 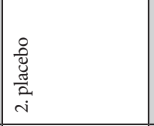 & 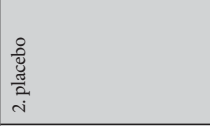 & 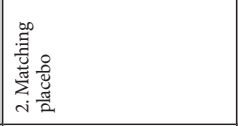 & 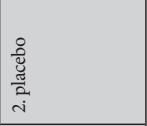 & 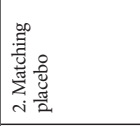 & 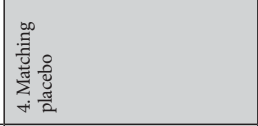 & 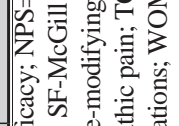 \\
\hline 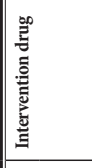 & 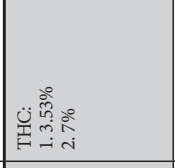 & 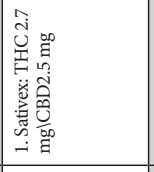 & 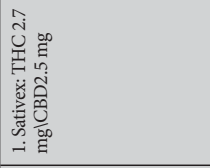 & 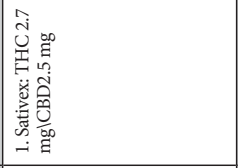 & 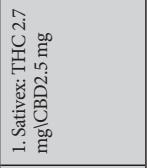 & 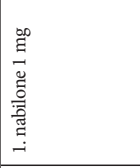 & 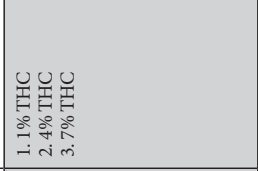 & 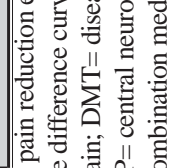 \\
\hline 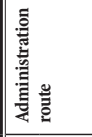 & 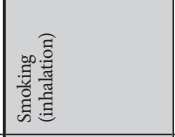 & 善 & 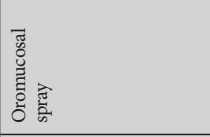 & 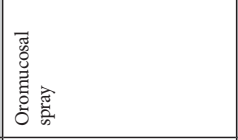 & 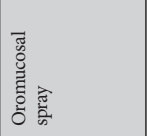 & 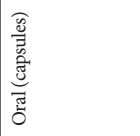 & 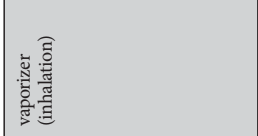 & 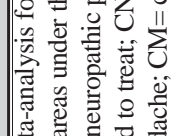 \\
\hline 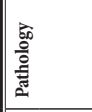 & $\hat{\mathrm{z}}$ & 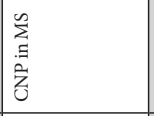 & $\begin{array}{l}\sum_{i} \\
\text { E } \\
\dot{z} \\
\end{array}$ & 言 & 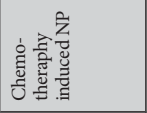 & 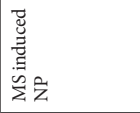 & ză & 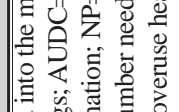 \\
\hline 言 & 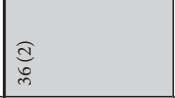 & 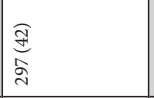 & $\underset{\Xi}{\exists}$ & 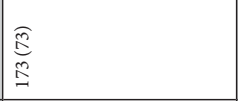 & $\begin{array}{l}0 \\
0 \\
0 \\
0\end{array}$ & $\underbrace{E}_{ \pm}$ & 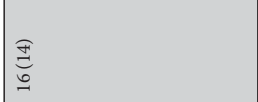 & 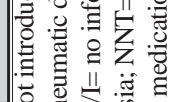 \\
\hline 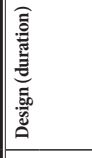 & 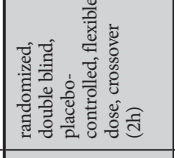 & 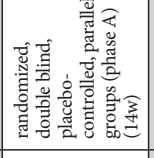 & 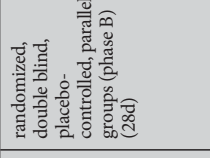 & 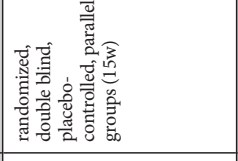 & 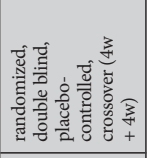 & 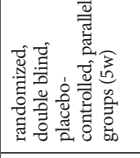 & 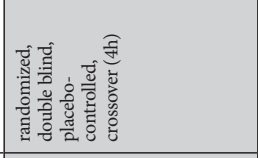 & 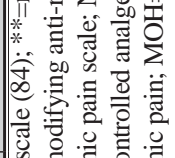 \\
\hline 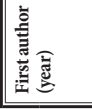 & 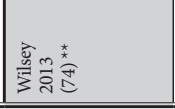 & 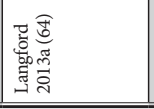 & 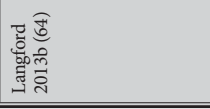 & 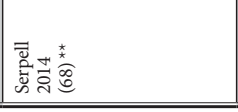 & 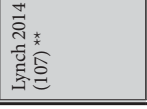 & 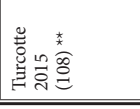 & 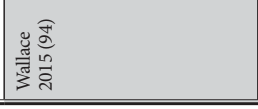 & 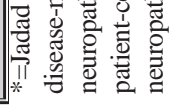 \\
\hline
\end{tabular}




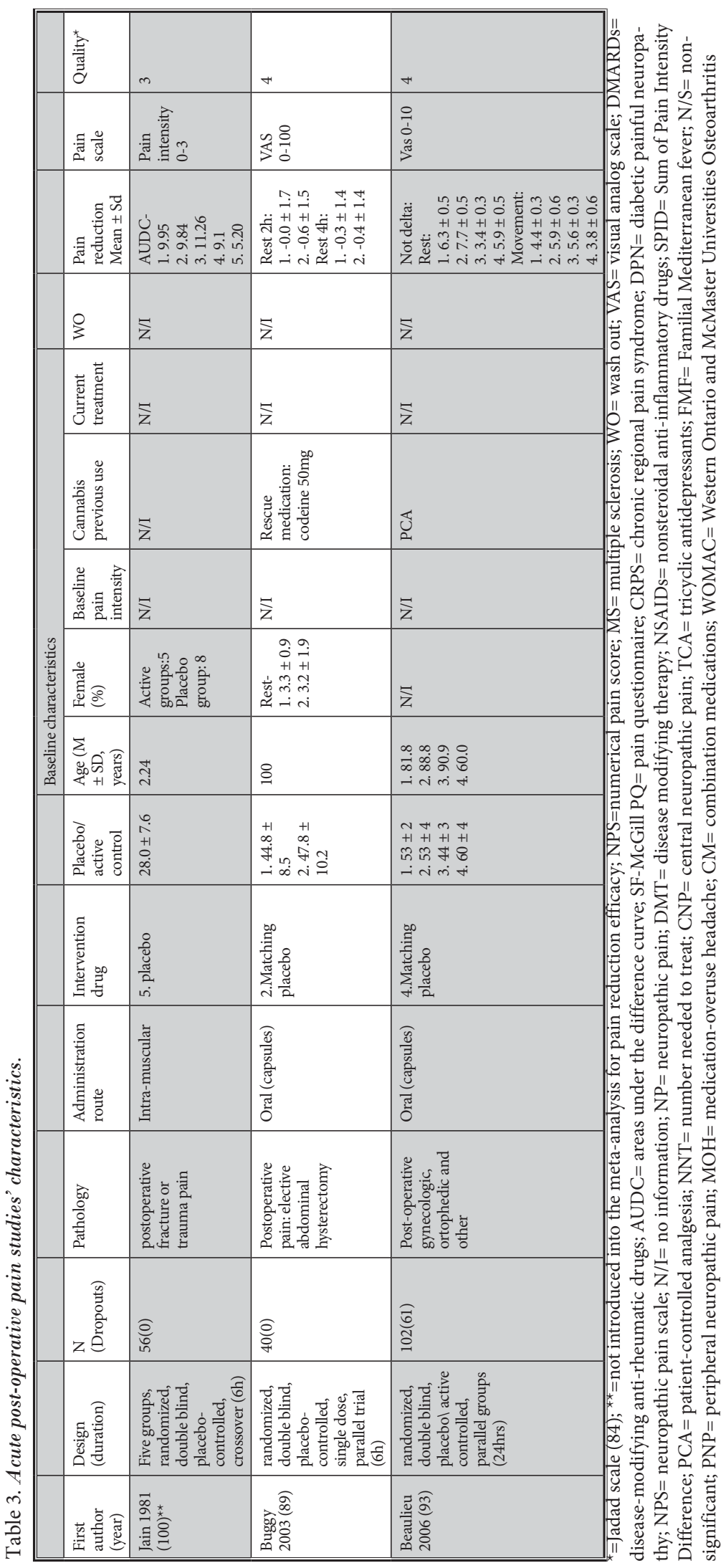

effects of CBMs vs. placebo in these trials and the small number of trials conducted on postoperative pain, which would not be comparable to the trials on chronic pain (Fig. 2). Furthermore, in 2 of the studies that were included in our metaanalysis $(47,48)$, pain reduction was not the primary outcome for the study and was not measured for the entire sample.

In addition, 3 of the studies that were meta-analyzed in the primary analysis included only an intervention group of an active drug, utilizing analgesic medication (dihydrocodeine, ibuprufen, or diphenhydramine), as so, they were redacted in the following analysis because of their analgesic effect that may interfere with the comparisons between "real" placebo to CBMs (Fig. 3) $(90,91,95)$. Two other studies that were meta-analyzed had an analgesic medication (codeine or secobarbital) as one or more of the study arms as well as to placebo; however, these "comparator" arms were not included in any of the analyses $(57,60)$.

Additionally, 5 studies that were reviewed included inhalation/vaporization of cannabinoids $(72-74,94,96)$ with several concentration arms/groups compared to placebo, i.e., $0 \%$ THC, but only 3 of them $(72,73,94)$, for reasons that will be explained in section 3.7, were metaanalyzed with the overall RCTs (Fig. 1) and separately (Fig. 4). In the past, this manner of administration has been either ignored or deliberately left out for various reasons (40), although evidence points to inhalation as the preferred route for rapid and effective cannabinoid use $(12,14)$.

\section{Summary of Measures and Synthesis of Results}

All analyses were performed using the Comprehensive Meta-Analysis (CMA) Version 3 software (Biostat, Englewood, NJ) (97). For pain reduction efficacy, a few outcome measures were used in the current meta-analyses. Eighteen of the 24 included studies, which reported results by each intervention group's initial 
Effective of Cannabis-Based Medicines for Pain Management

Table 4. Jadad scale ratings.

\begin{tabular}{|c|c|c|c|c|c|}
\hline $\begin{array}{c}\text { Score } \\
\text { criteria } \\
\text { First author, year }\end{array}$ & $\begin{array}{c}\text { Point for } \\
\text { randomization }\end{array}$ & $\begin{array}{l}\text { Point for double } \\
\text { blind design }\end{array}$ & $\begin{array}{c}\text { Point for dropouts } \\
\text { description }\end{array}$ & $\begin{array}{c}\text { Points for adequate } \\
\text { randomization and/or } \\
\text { appropriate blinding }\end{array}$ & $\begin{array}{l}\text { Total } \\
\text { score }\end{array}$ \\
\hline Noyes, 1975a (57) & 1 & 1 & 0 & 0 & 2 \\
\hline Noyes, 1975b (56) & 1 & 1 & 0 & 0 & 2 \\
\hline Staquet, 1978a (60) & 1 & 1 & 1 & 0 & 3 \\
\hline Staquet, 1978b (60) & 1 & 1 & 1 & 0 & 3 \\
\hline Jochimsen, $1987(53)^{\star}$ & 1 & 1 & 1 & 1 & 4 \\
\hline Johnson, 2010 (63) & 1 & 1 & 1 & 1 & 4 \\
\hline Portenoy, $2012(67)^{*}$ & 1 & 1 & 1 & 1 & 4 \\
\hline Maurer, $1990(101)^{*}$ & 1 & 1 & 0 & 1 & 3 \\
\hline Holdcroft, $1997(102)^{*}$ & 1 & 1 & 1 & 0 & 3 \\
\hline Karst, $2003(44)^{*}$ & 1 & 1 & 1 & 2 & 5 \\
\hline Wade, 2003 (47) & 1 & 1 & 1 & 1 & 4 \\
\hline Notcutt, $2004(55)^{*}$ & 1 & 1 & 1 & 1 & 4 \\
\hline Berman, 2004 (43) & 1 & 1 & 1 & 1 & 4 \\
\hline Wade, 2004 (48) & 1 & 1 & 1 & 0 & 3 \\
\hline Svendsen, $2004(46)^{*}$ & 1 & 1 & 1 & 2 & 5 \\
\hline Rog, 2005 (45) & 1 & 1 & 1 & 2 & 5 \\
\hline Salim, $2005(103)^{*}$ & 1 & 1 & 1 & 1 & 4 \\
\hline Wissel, 2006 (61) & 1 & 1 & 1 & 0 & 3 \\
\hline Blake, 2006 (52) & 1 & 1 & 1 & 0 & 3 \\
\hline Abrams, $2007(62)^{*}$ & 1 & 1 & 1 & 2 & 5 \\
\hline Nurmikko, 2007 (65) & 1 & 1 & 1 & 2 & 5 \\
\hline Frank, 2008 (90) & 1 & 1 & 1 & 2 & 5 \\
\hline Skrabek, $2008(59)^{*}$ & 1 & 1 & 1 & 1 & 4 \\
\hline Wilsey, 2008 (73) & 1 & 1 & 1 & 1 & 4 \\
\hline Narang, $2008(104)^{*}$ & 1 & 1 & 1 & 1 & 4 \\
\hline Ellis $2009(71)^{*}$ & 1 & 1 & 1 & 1 & 4 \\
\hline Ware, $2010(72)$ & 1 & 1 & 1 & 1 & 4 \\
\hline Selvarajah, 2010 (66) & 1 & 1 & 1 & 0 & 3 \\
\hline Toth, $2012(92)$ & 1 & 1 & 1 & 1 & 4 \\
\hline Corey-Bloom, $2012(96)^{*}$ & 1 & 1 & 1 & 1 & 4 \\
\hline Pini, 2012 (95) & 1 & 1 & 1 & 1 & 4 \\
\hline Huggins, $2012(105)^{\star}$ & 1 & 1 & 1 & 1 & 4 \\
\hline Zajicek, $2012(106)^{*}$ & 1 & 1 & 1 & 0 & 3 \\
\hline Wilsey, $2013(74)^{*}$ & 1 & 1 & 1 & 1 & 4 \\
\hline Langford, 2013a (64) & 1 & 1 & 1 & 2 & 5 \\
\hline Langford, 2013b (64) & 1 & 1 & 1 & 2 & 5 \\
\hline Serpell, $2014(68)^{*}$ & 1 & 1 & 1 & 2 & 5 \\
\hline Lynch, $2014(107)^{*}$ & 1 & 1 & 1 & 0 & 3 \\
\hline Turcotte $2015(108)^{*}$ & 1 & 1 & 1 & 2 & 5 \\
\hline Wallace, 2015 (94) & 1 & 1 & 1 & 1 & 4 \\
\hline Jain, $1981(100)^{*}$ & 1 & 1 & 1 & 0 & 3 \\
\hline Buggy, 2003 (89) & 1 & 1 & 1 & 1 & 4 \\
\hline Beaulieu, 2006 (93) & 1 & 1 & 1 & 1 & 4 \\
\hline
\end{tabular}

${ }^{*}=$ not introduced into the meta-analysis for pain reduction efficacy 


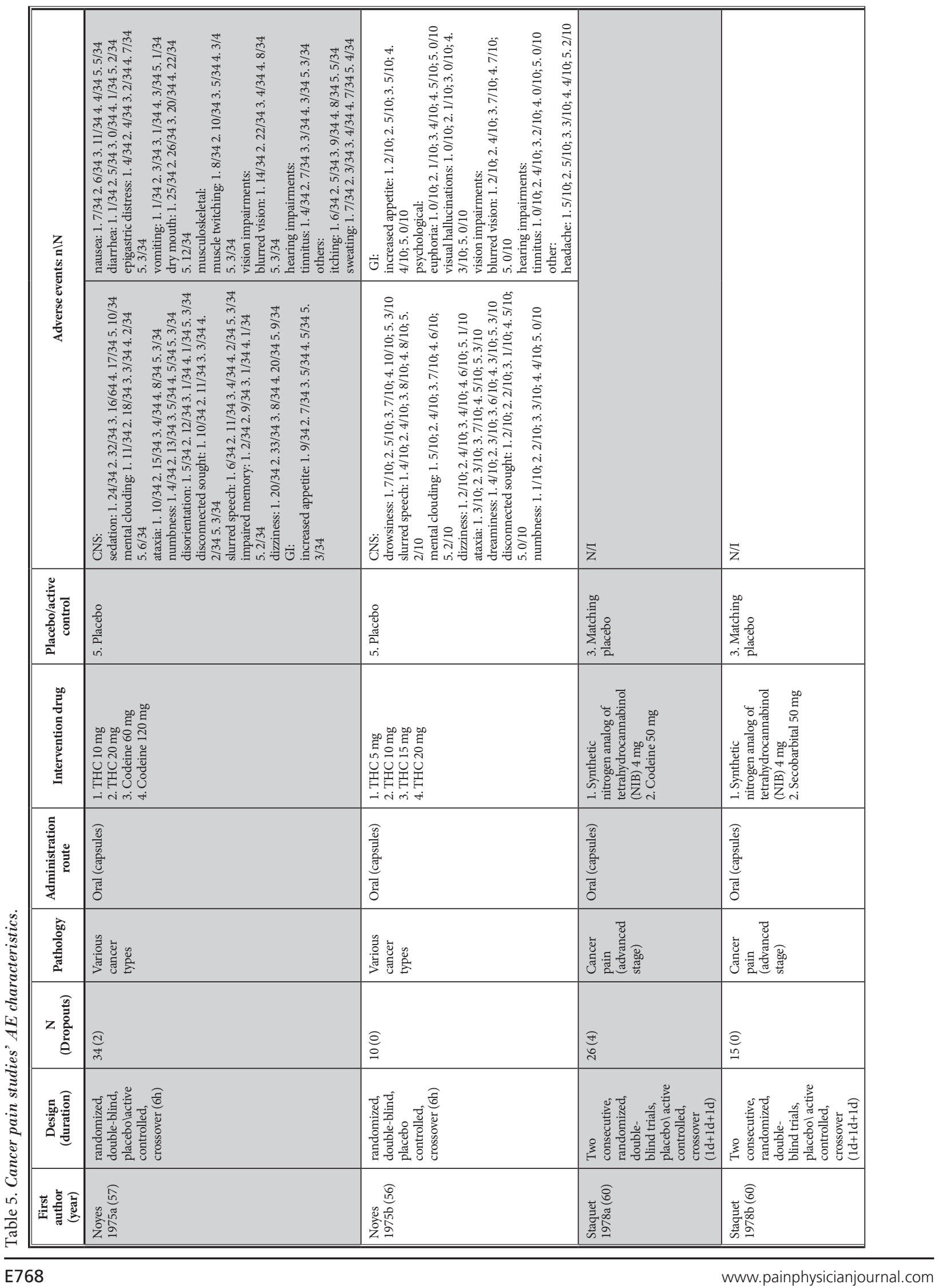




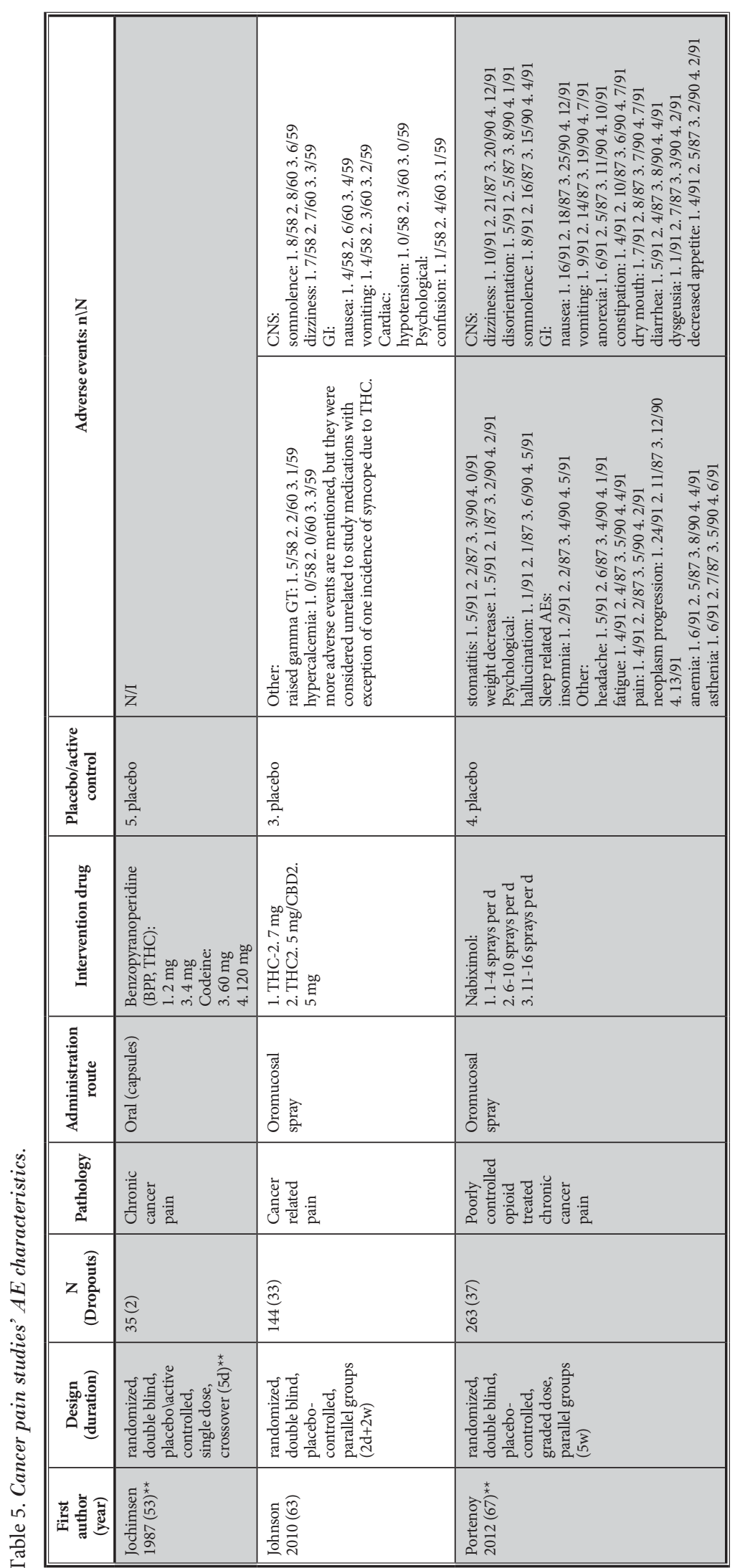

and final means and standard deviations (SD) difference from baseline separately for the intervention and for the placebo groups, measured on pain scales and quantified in the same direction. Based on these, the software consequently calculated the SD difference in means, standard error, and Hedges's g scores for each of these studies.

Some of the studies did not include in their report those raw results but rather reported their results as difference in means, confidence interval of $95 \%$ lower, and upper limits and the sample size; this was the case in 6 studies with a crossover design and 5 studies with a parallel design. For AEs, risk ratios with a $95 \%$ confidence interval were computed separately for each categorical system of AEs.

All the analyses performed are presented in the figures by fitting both fixed and random effects models. Heterogeneity between studies was statistically studied using the $I^{2}$ statistic and $\mathrm{Tau}^{2}$ tests (98).

Due to insufficient reporting on raw data or due to inadaptable reporting of results, some of the studies that were reviewed were found as incompatible for the metaanalysis and therefore they were redacted from our study (as indicated in both Table 1-3 and Table 5-7). In the cases of the studies that investigated more than one cannabinoid preparation versus placebo, the comparisons were made separately and they were analyzed as separate studies; this should not have caused any 
Pain Physician: September/October 2017: 20:E755-E796

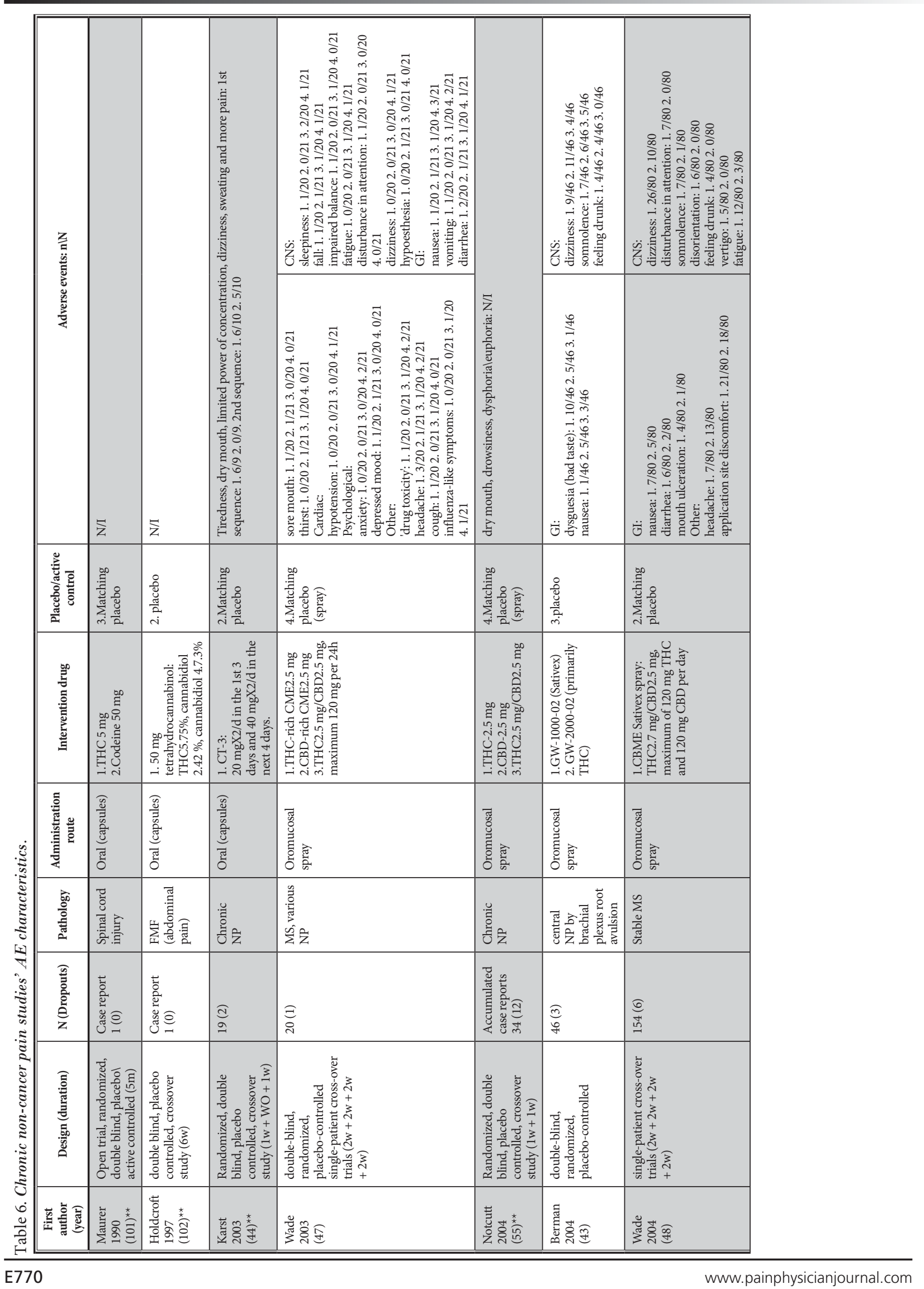


Effective of Cannabis-Based Medicines for Pain Management

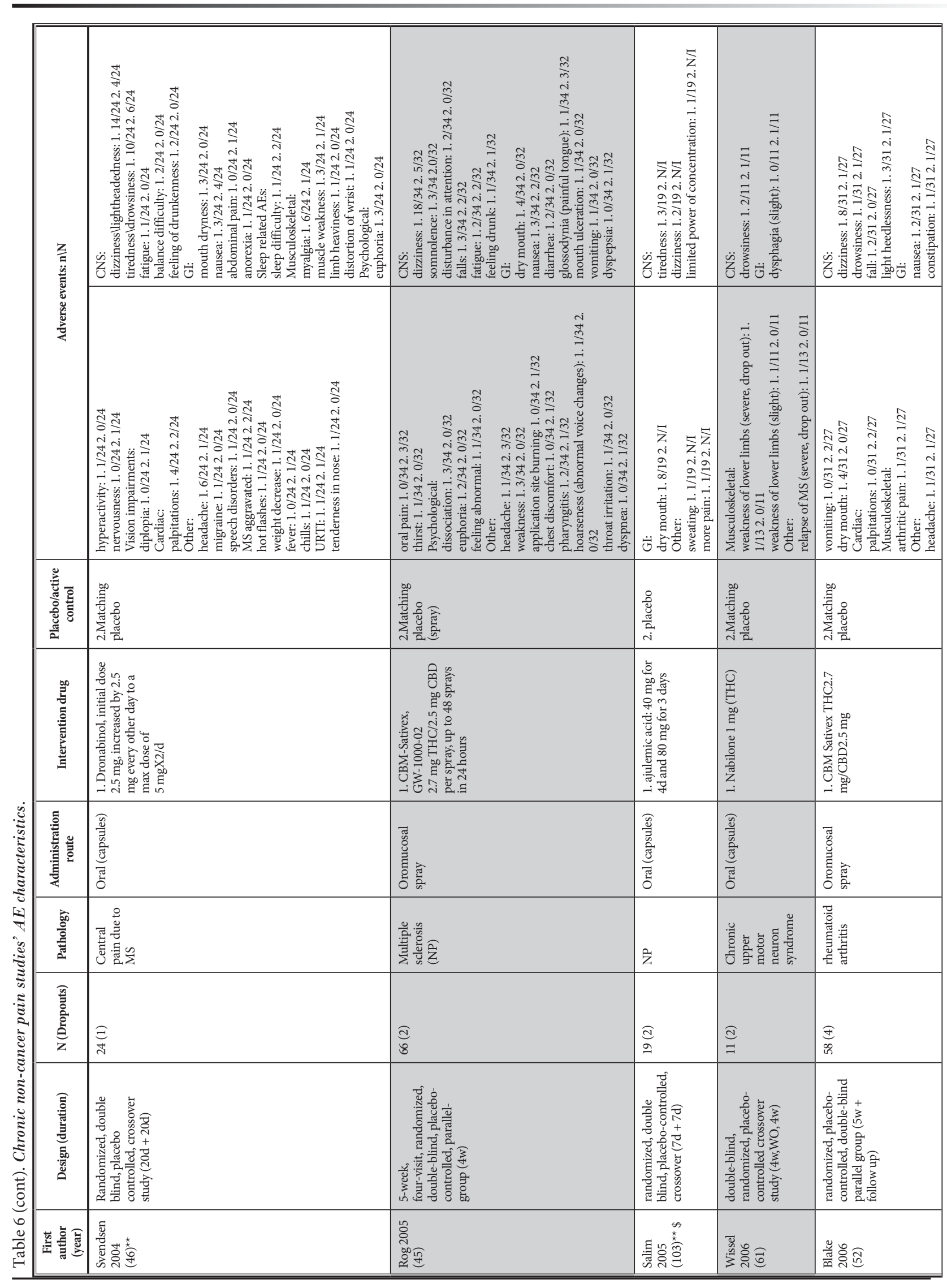


Pain Physician: September/October 2017: 20:E755-E796

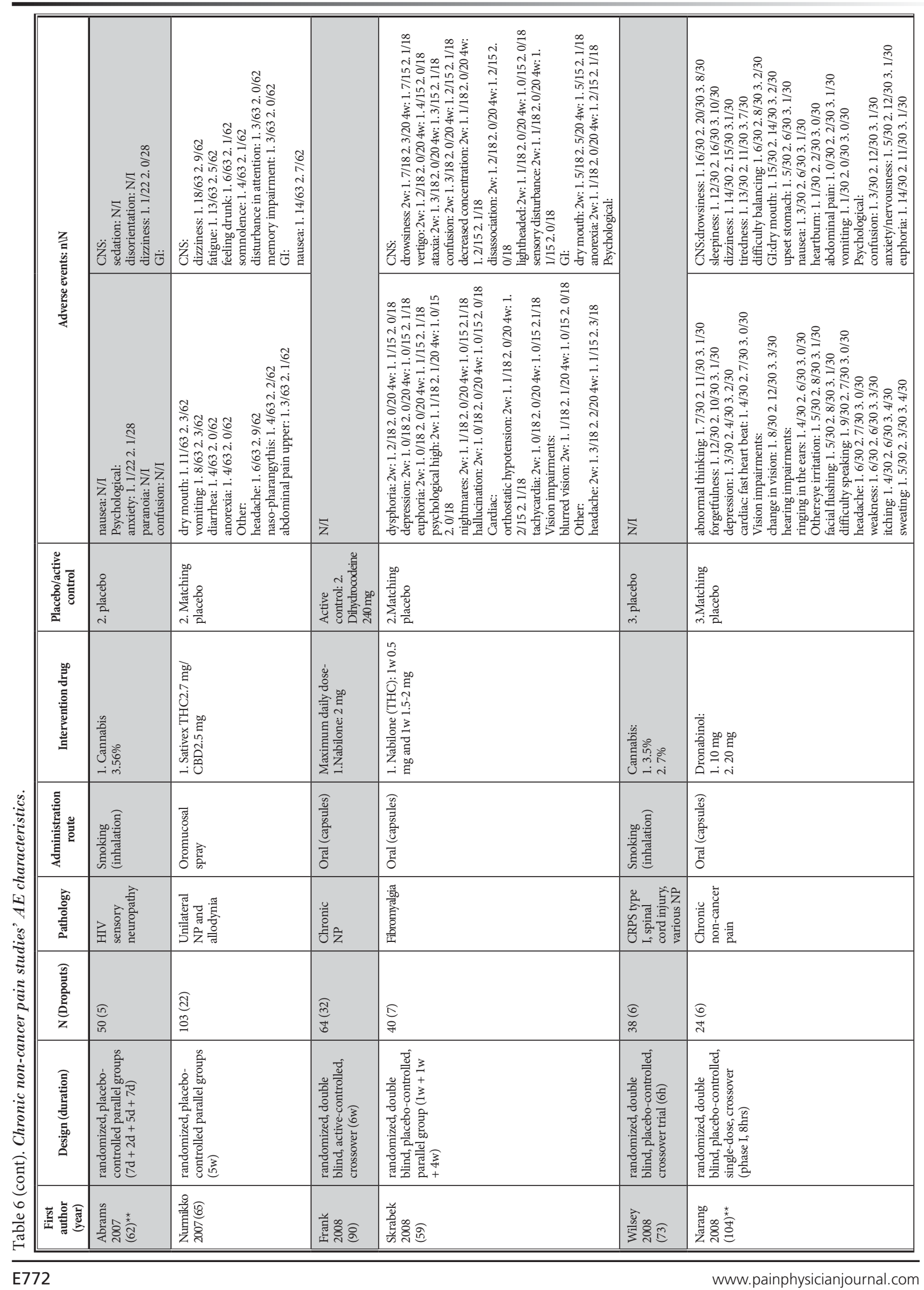


Effective of Cannabis-Based Medicines for Pain Management

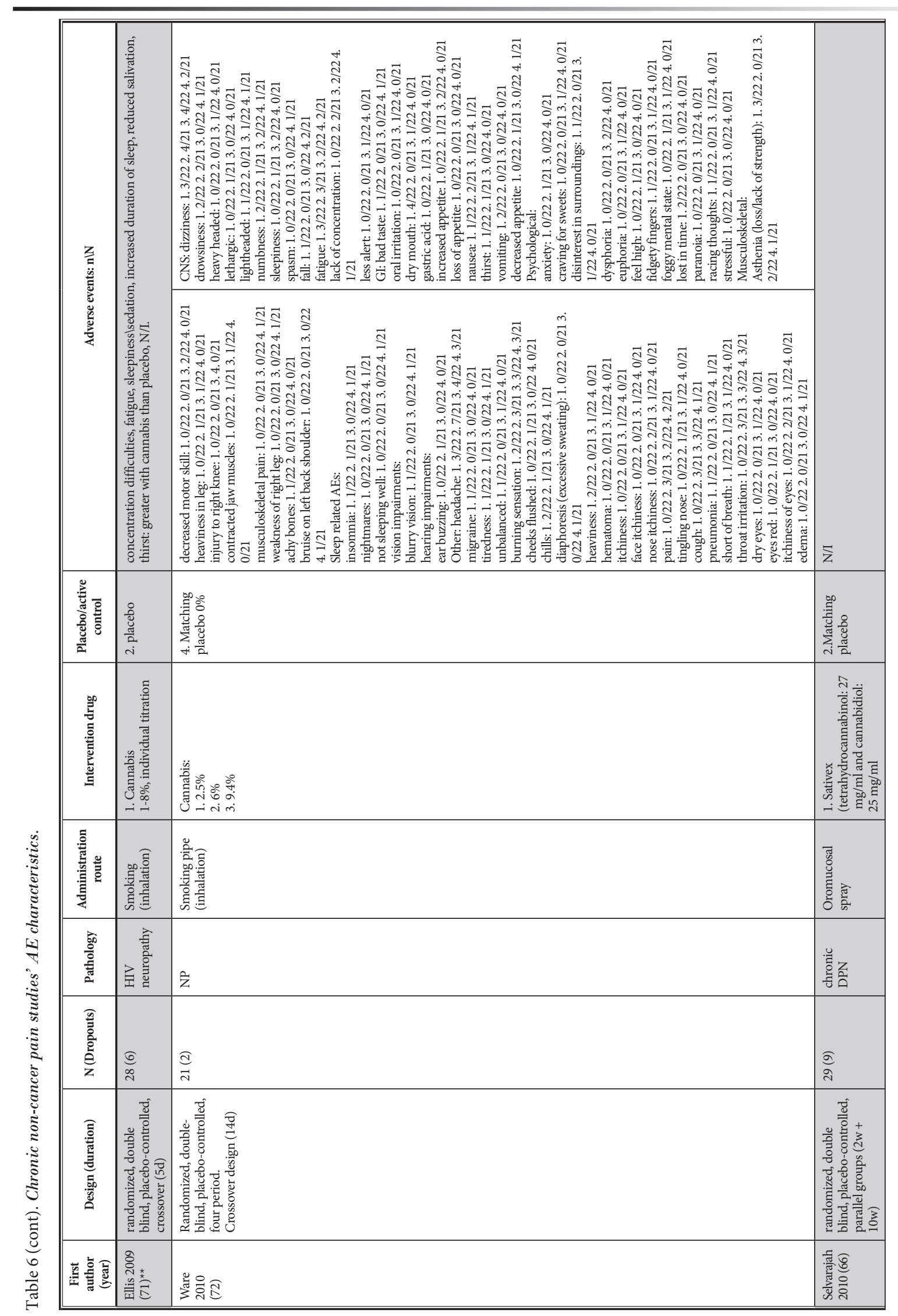


Pain Physician: September/October 2017: 20:E755-E796

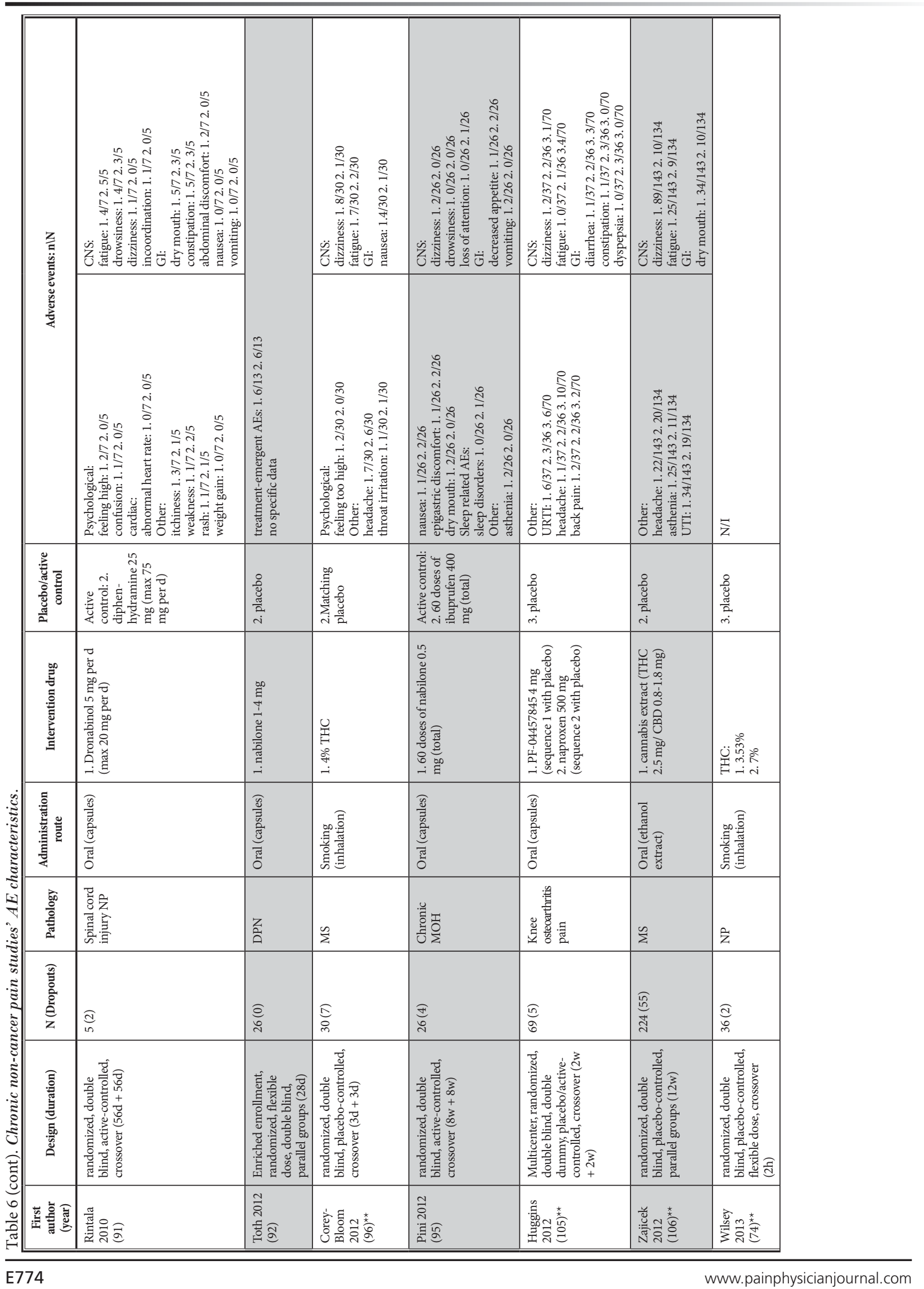


Effective of Cannabis-Based Medicines for Pain Management

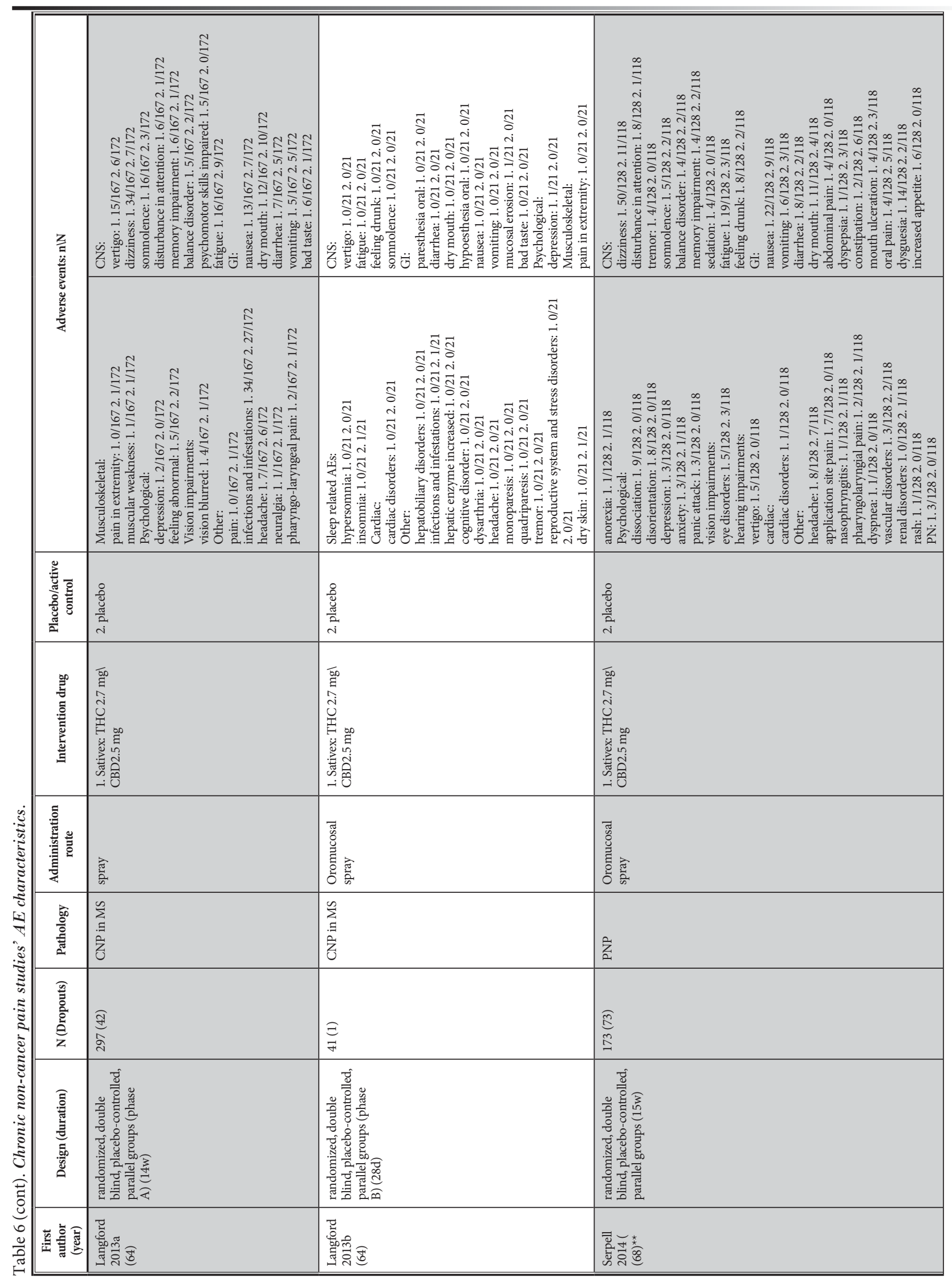




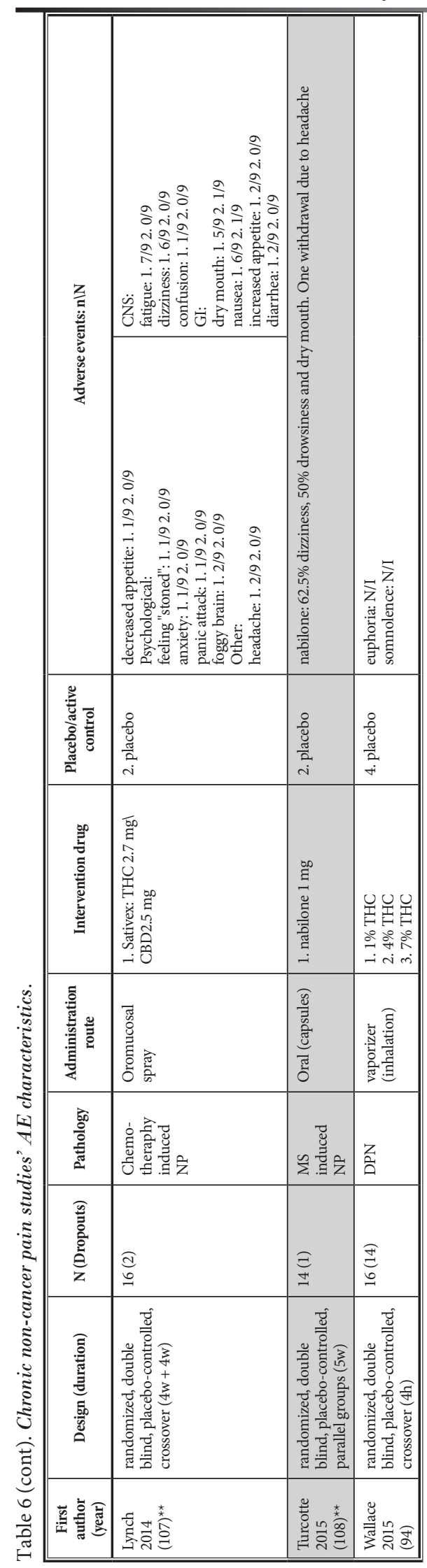

limitations to the overall results due to the doubleblinding procedure of these trials. No additional analyses were made.

\section{Risk of Limitations across Studies}

An important consideration for limitation across the studies is publication. Unpublished studies could have given our study more evidence for either direction, but this would have come on the expense of our study's reliability. Furthermore, the decision to include only studies that were published in English may have caused publication limitation as well. Specifically, 2 studies that were included in the former review/meta-analysis were not published in English, thereby, they could not be included $(58,99)$.

Furthermore, from the studies included in the efficacy meta-analysis, only 2 did not report any information regarding the analgesic treatments permitted during the intervention period $(57,89)$. Moreover, only 7 studies reported on the patients' history of prior consumption of CBMs (Tables 1-3) $(45,52,59,65,67,72,92)$.

\section{RESULTS}

\section{Study Selection}

The flow chart of studies through selection process is presented in Fig. 5. The electronic and manual search yielded 1,126 titles of studies that examined the effects of CBMs on pain from 1975 to July of 2015. Inspection of the abstracts led to 972 of these studies to be discarded. As a result, 154 full text studies were reviewed. Unpublished or poster-based data were not included or analyzed in this review due to the need for valid, peer-reviewed data. An additional 111 studies were excluded for various reasons: 78 studies failed to meet the requirement of RCT standards, 15 studies were conducted on healthy volunteers, and 18 studies had an open-label design.

We ended up including the 42 studies which fulfilled our inclusion criteria for this review (4348,52,53,55-57,59-68,71-74,89-96,100-108) (Tables 1-3 and 5-7). However, the meta-analysis section of this study had much more specific requirements. Of the 42 studies that were reviewed for the efficacy evaluation of CBMs, 19 had been redacted. Fifteen studies used outcome measures that did not fit to any form of analysis in the CMA software $(44,46,53$, $62,67,68,71,74,96,100,103-108)$. An additional 2 studies were case reports of RCTs $(101,102)$, one reported on the results of accumulated case reports 
of RCTs (55), and one study was redacted because the different intervention groups did not report similar baseline pain intensity scores, which may have led to a limitation in the results (44). Consequently, 24 studies were included in the meta-analyses for the examination of the efficacy of CBMs for pain reduction. Of these, 5 studies $(43,60,89,93,109)$ had more than one arm of intervention that was included in the meta-analysis. Study characteristics are presented in Tables 1-3.

\section{Study Quality and Risk of Limitations within Studies}

A summary of the methodological quality and level of evidence for each study is shown in tables 1-3. Some studies displayed flaws with respect to control of selection limitation. In most cases, no information was provided regarding the concealment allocation process or the method of randomization $\quad(43,44,46-$ $48,52,53,55-57,59$ $61,66,74,89,91,93,100$ 102). This phenomenon, which is usual in small trials, increases the risk of possible selection limitation (110). All of the studies were conducted on a double-blind basis; however, the adequacy of blinding was not tested in any of the trials.

Since cannabis is a substance surrounded by considerable controversy in

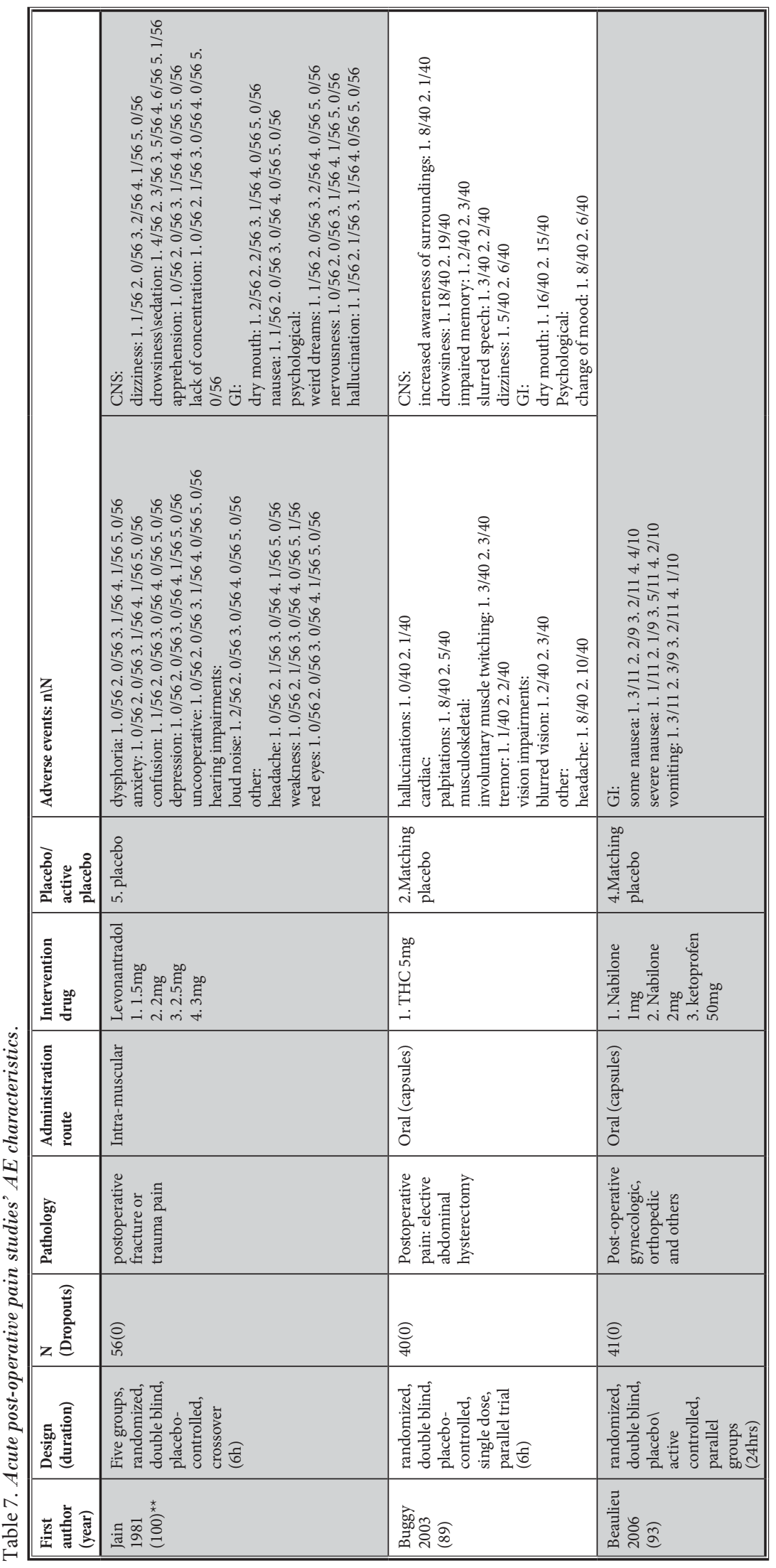

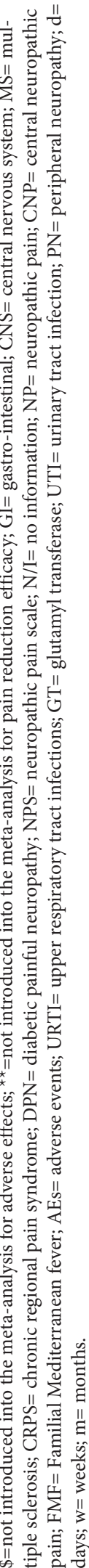


Pain Physician: September/October 2017: 20:E755-E796

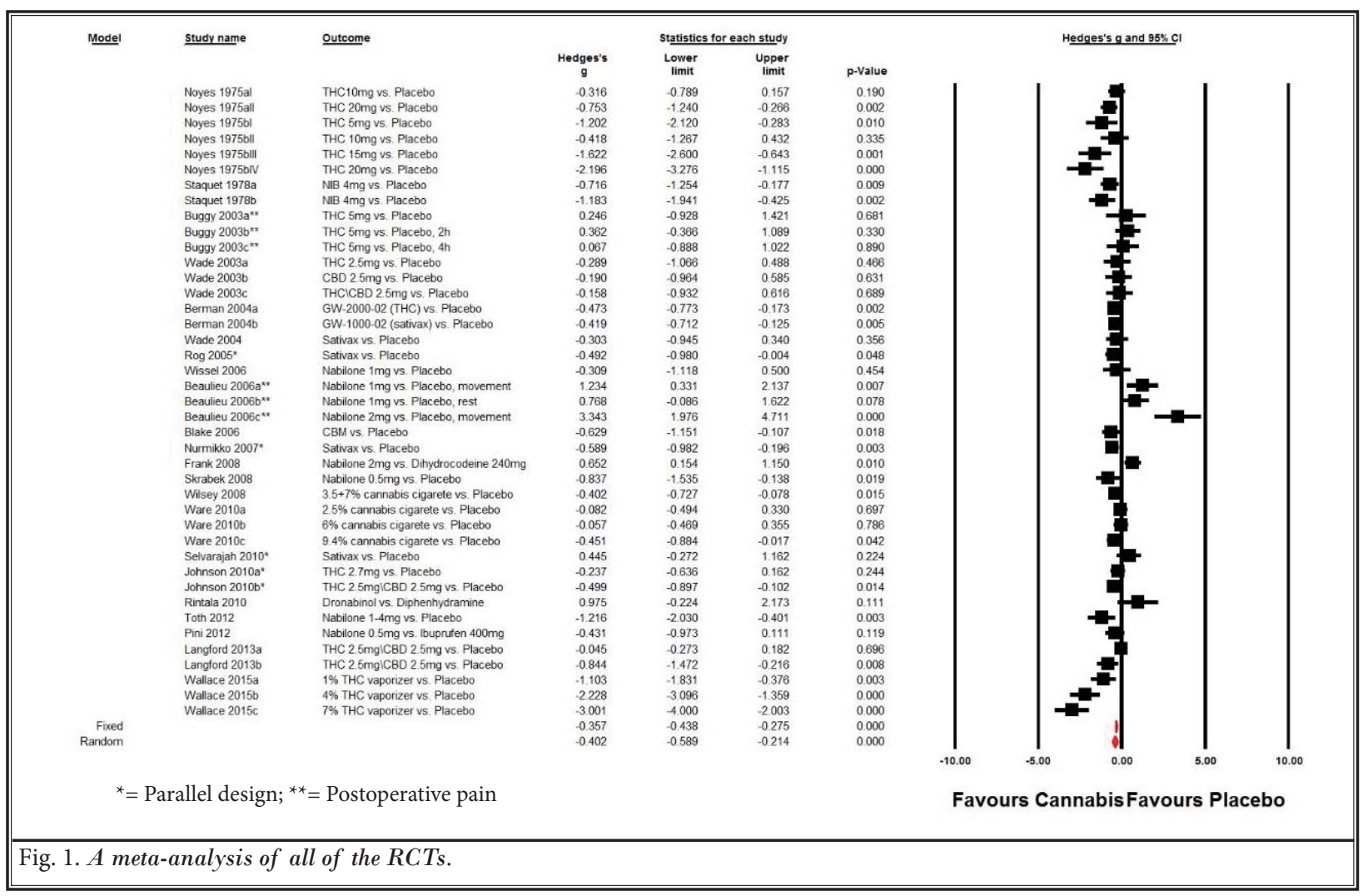

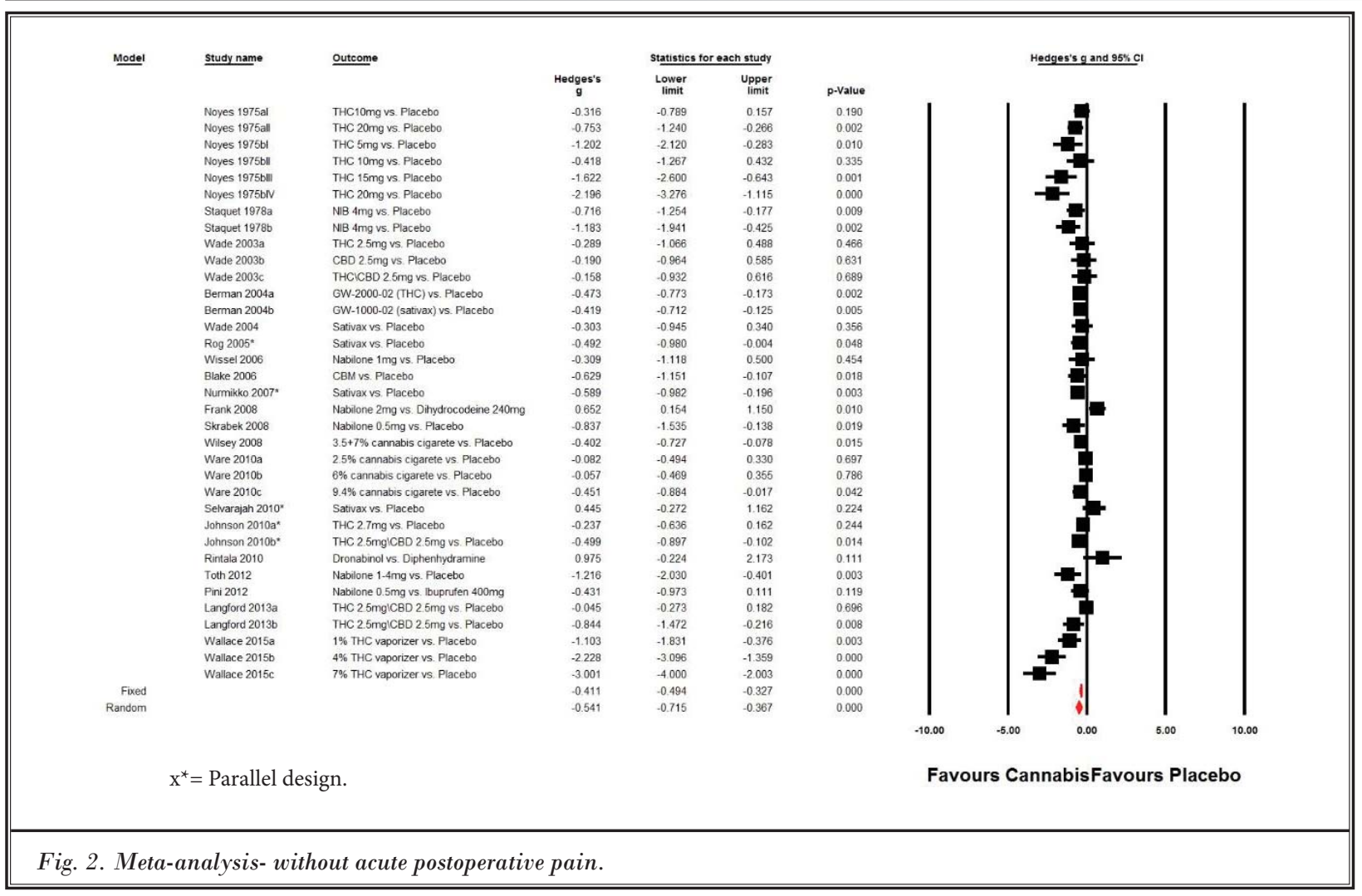


Effective of Cannabis-Based Medicines for Pain Management

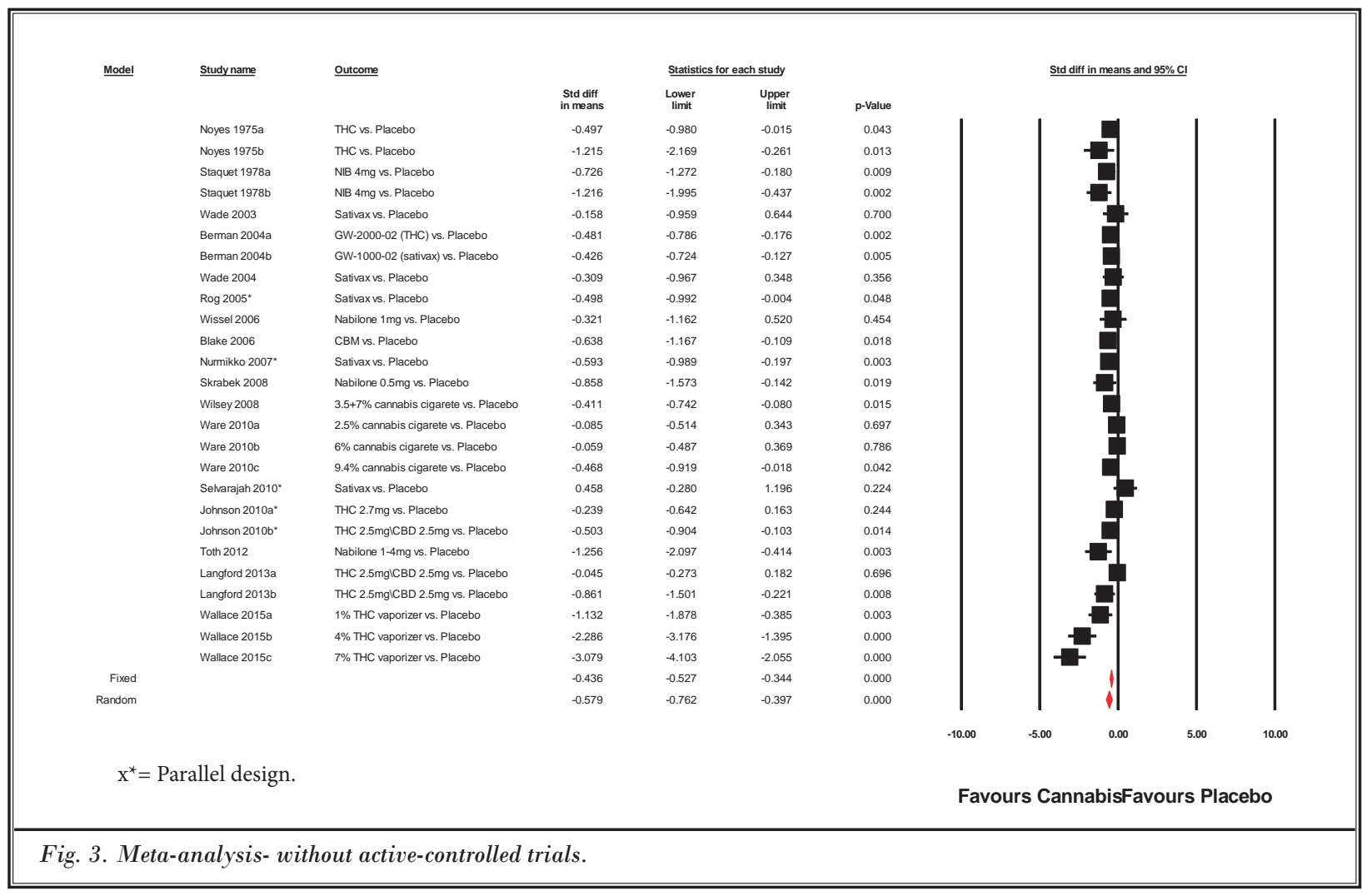

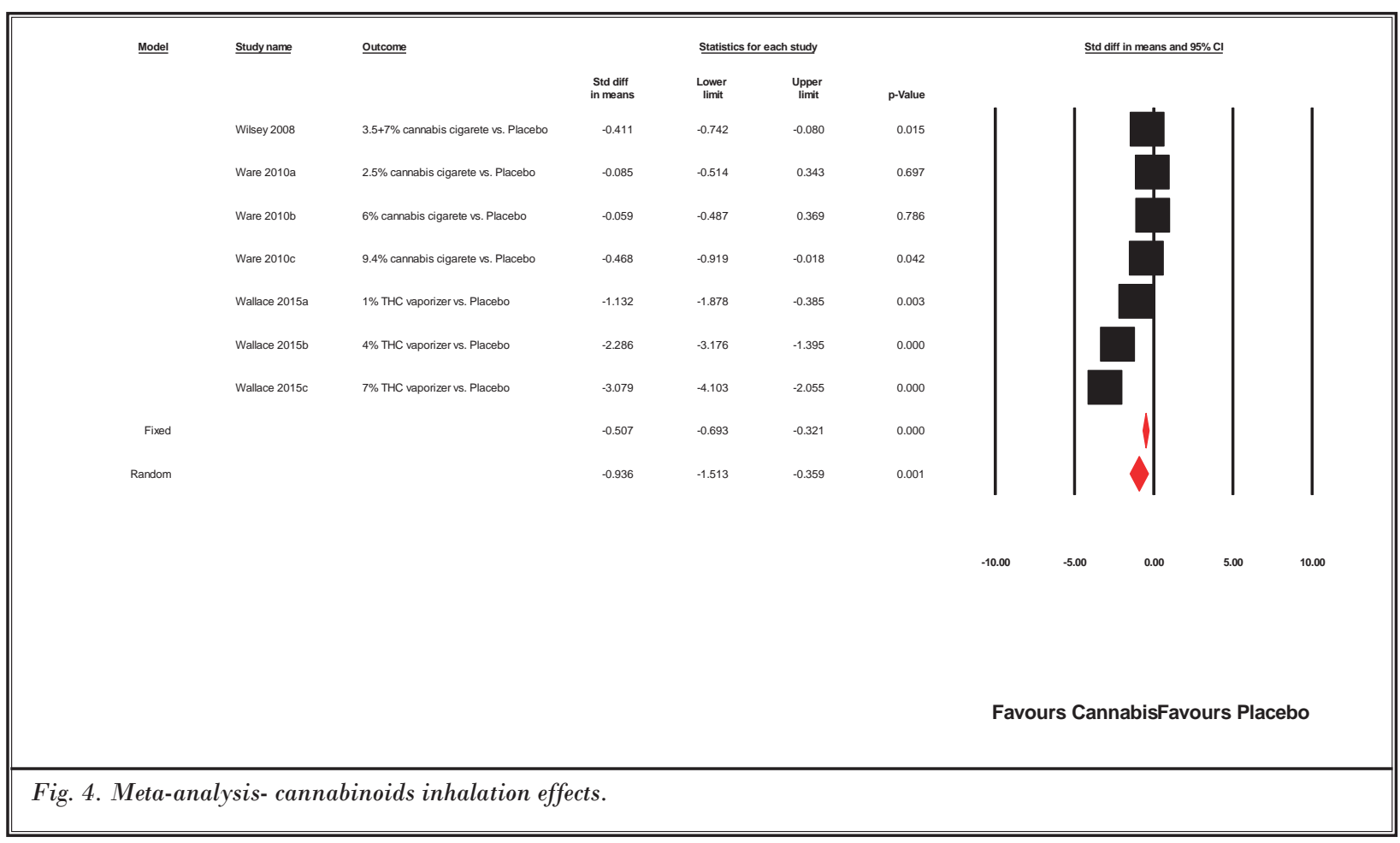

www.painphysicianjournal.com 
the media and in society, cannabinoids have a marked placebo effect; therefore, inadequate blinding would constitute an important source of limitation in these types of studies. Additionally, the characteristic side effects caused by these substances render perfect masking extremely difficult. Moreover, some of the studies did not note that they used matching placebo (same appearance), but rather plain placebo $(43,56,57,63,73,92)$, which can misattribute to the masking procedure considerably, especially due to the unusual taste and smell of cannabinoids. Some studies even used an active drug as an active control $(90,91,95)$.

Another important flaw in terms of study quality lay in the control of attrition limitation. In only 8 of the studies there were no losses or withdrawals of subjects $(56,60,89,92,93,100-102)$, while only 6 studies specified that results had been analyzed on an intention to treat basis $(43,45,52,64,65,109)$.

Lastly, studies varied considerably in outcome assessment and reporting approaches. Specifically, several studies expressed data as median values $(46,52,55,71)$, with only reporting means without standard deviations $(43,46,48,59,65,71,73,96,100,102,109)$, as areas under the difference curve (AUDC) (100), as sum of pain intensity difference (SPID) (104), as pain reduction in $\mathrm{N} \%$ (percentage of subjects that reported pain reduction) (53), as the number of the subjects with $30 \%$ and/or $50 \%$ pain reduction $(55,64,71,74,103)$, or as non-quantitative data where pain reduction is shown only on a graph, with no raw data (62).

\section{Results of Individual Studies}

The results of individual studies are presented in particulars in Tables 1-3.

\section{Synthesis of Results}

\section{Meta-Analysis of All Included RCTs}

In measures of change from baseline pain intensity, the overall effect size of the included 24 crossover and parallel design RCTs (and some of their intervention arms) that examined the effect of CBMs on pain registered standardized mean differences (SMD). For a fixed-effect model of -0.35 Hedge's g $(-0.43$ to -0.27 , $P<0.0001)$ and for a random-effect model of -0.40 Hedge's $g(-0.58$ to $-0.21, P<0.0001)$, both were found favorable towards CBMs over placebo. Not all of the studies yielded results in the same direction, and a statistical heterogeneity was in evidence $(I 2=77.83 \%, P<$ $0.0001)$, which represents that the dispersion is due to real dispersion in the effect sizes of the studies (Tau2 = 0.25, Tau $=0.50$ ) (Fig. 1).

\section{Meta-Analysis- Without Active-Controlled Trials}

Because active control can be regarded as a more efficient analgesic than real placebo, a further reduction of those 3 studies $(90,91,95)$ was made from the meta-analysis in order to get more precise results regarding the analgesic effects of CBMs. This analysis produced more benefit for CBMs over placebo: SMD for a fixed-effect model of -0.45 Hedge's g $(-0.54$ to -0.36 , $P<0.0001)$ and for a random-effect model of -0.61 Hedge's $g(-0.78$ to $-0.43, P<0.0001)$. Again, not all of the studies yielded results in the same direction, and statistical heterogeneity was in evidence $(12=70.12 \%$, $P<0.0001)$, which represents that the dispersion is due to real dispersion in the effect sizes of the studies (Tau2 $=0.15$, Tau $=0.39)($ Fig. 3).

\section{Meta-Analysis- Cannabinoids Inhalation Effects}

Although the most clinically common route of administration is by smoking/inhalation, only 3 studies that used this route of administration were meta-analyzed $(72,73,94)$. This analysis produced more benefit for CBMs over placebo: SMD for a fixed-effect model of -0.50 Hedge's g $(-0.69$ to $-0.32, P<0.0001)$ and for a random-effect model of -0.93 Hedge's g ( -1.51 to -0.35 , $P=0.001)$. However, in this analysis, all of the studies yielded results in the same direction, but a statistical heterogeneity was in evidence $(I 2=88.11 \%, P<0.0001)$, and a dispersion of Tau2 $=0.50$, Tau $=0.71$ (Fig. 4).

\section{Meta-Analysis- Cannabinoids Effects on Chronic NP}

In order to examine the effectiveness of CBMs for NP, a separate meta-analysis was made to the RCTs that examined NP directly. The studies that did examine NP, but used an active control $(90,91)$, were excluded in order to make a more precise comparison. Eleven RCTs qualified for this analysis. This analysis produced more benefit for CBMs over placebo: SMD for a fixed-effect model of -0.38 Hedge's $g(-0.48$ to $-0.27, P<0.0001)$ and for a randomeffect model of -0.52 Hedge's g $(-0.75$ to $-0.30, P<0.0001)$. However, in this analysis, all of the studies yielded results in the same direction, but there was a statistical heterogeneity in evidence $(I 2=75.70 \%, P<0.0001)$, and a dispersion of Tau2 $=0.16$, Tau $=0.41$ (Fig. 6). 
Effective of Cannabis-Based Medicines for Pain Management

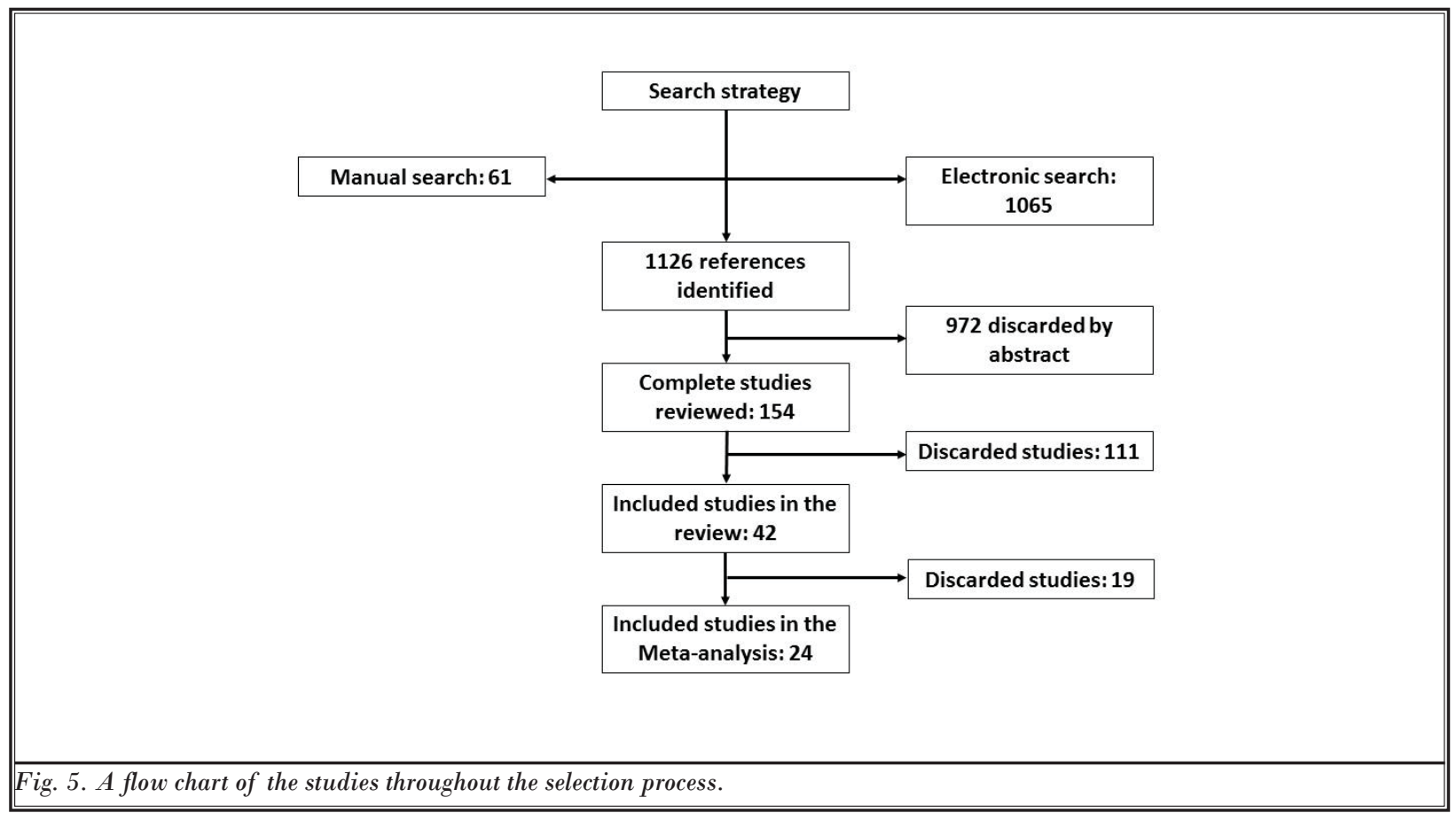

\section{Meta-Analysis- Cannabinoids Effects on Cancer Pain}

In order to examine the effects of CBMs on cancer pain, a separate meta-analysis was made to the RCTs that examined cancer pain directly. The 2 studies that did examine cancer pain but could not be used in the meta-analysis, for previously mentioned reasons in section 3.7, were excluded (Table 1). Three RCTs (10 arms) qualified for this analysis. This analysis produced more benefit for CBMs over placebo: SMD for a fixed-effect model of -0.62 Hedge's g $(-0.80$ to $-0.44, P<0.0001)$ and for a random-effect model of -0.76 Hedge's g $(-1.06$ to $-0.45, P<0.0001)$. In this analysis, all of the studies yielded results in the same direction, but a statistical heterogeneity was in evidence $(12=59.0 \%, P<0.01)$, and a dispersion of Tau2 $=0.12$, Tau $=0.35$ (Fig. 7).

\section{Meta-Analysis- Cannabinoids Effects on Chronic Non-Cancer Pain}

In order to examine the effects of CBMs on chronic non-cancer pain, a separate meta-analysis was made for the RCTs that examined chronic non-cancer pain directly. The 17 studies that did examine chronic non-cancer pain, but could not be used in the meta-analysis for previously mentioned reasons in section 3.7, were excluded (Table 2). Fourteen RCTs ( 22 arms) qualified for this analysis. This analysis produced more benefit for CBMs over placebo: SMD for a fixed-effect model of -0.39 Hedge's g ( -0.49 to $-0.29, P<0.0001)$ and for a random-effect model of -0.53 Hedge's g $(-0.75$ to $-0.32, P<0.0001)$. However, in this analysis, all of the studies yielded results in the same direction, but there was a statistical heterogeneity in evidence $(I 2=72.56 \%, P<0.0001)$, and a dispersion of Tau2 $=0.15$, Tau $=0.39$ (Fig. 8 ).

\section{Meta-Analysis- Cannabinoids Effects on Acute Postoperative Pain}

In order to examine the effects of CBMs on acute postoperative pain, a separate meta-analysis was made for the RCTs that examined acute postoperative pain directly. The one study that examined acute postoperative pain, but could not be used in the meta-analysis for previously mentioned reasons in section 3.7, was excluded (Table 3). Three RCTs (7 arms) were qualified for this analysis, which produced opposite direction results, where placebo produced a higher benefit over CBMs. The analysis produced a SMD for a fixed-effect model of 0.81 Hedge's g (0.41 to $1.21, P<0.0001)$ and for a random-effect model of 0.96 Hedge's g (0.16 to 1.76, $P<0.05)$. In this analysis, all of the studies yielded results in the same direction, but there was a statistical homogeneity in evidence $(12=72.99 \%, P<0.05)$, and a dispersion of Tau2 $=0.70$, Tau $=0.84$ (Fig. 9).

\section{REview}

In reviewing the 43 RCTs that were included in the 
Pain Physician: September/October 2017: 20:E755-E796

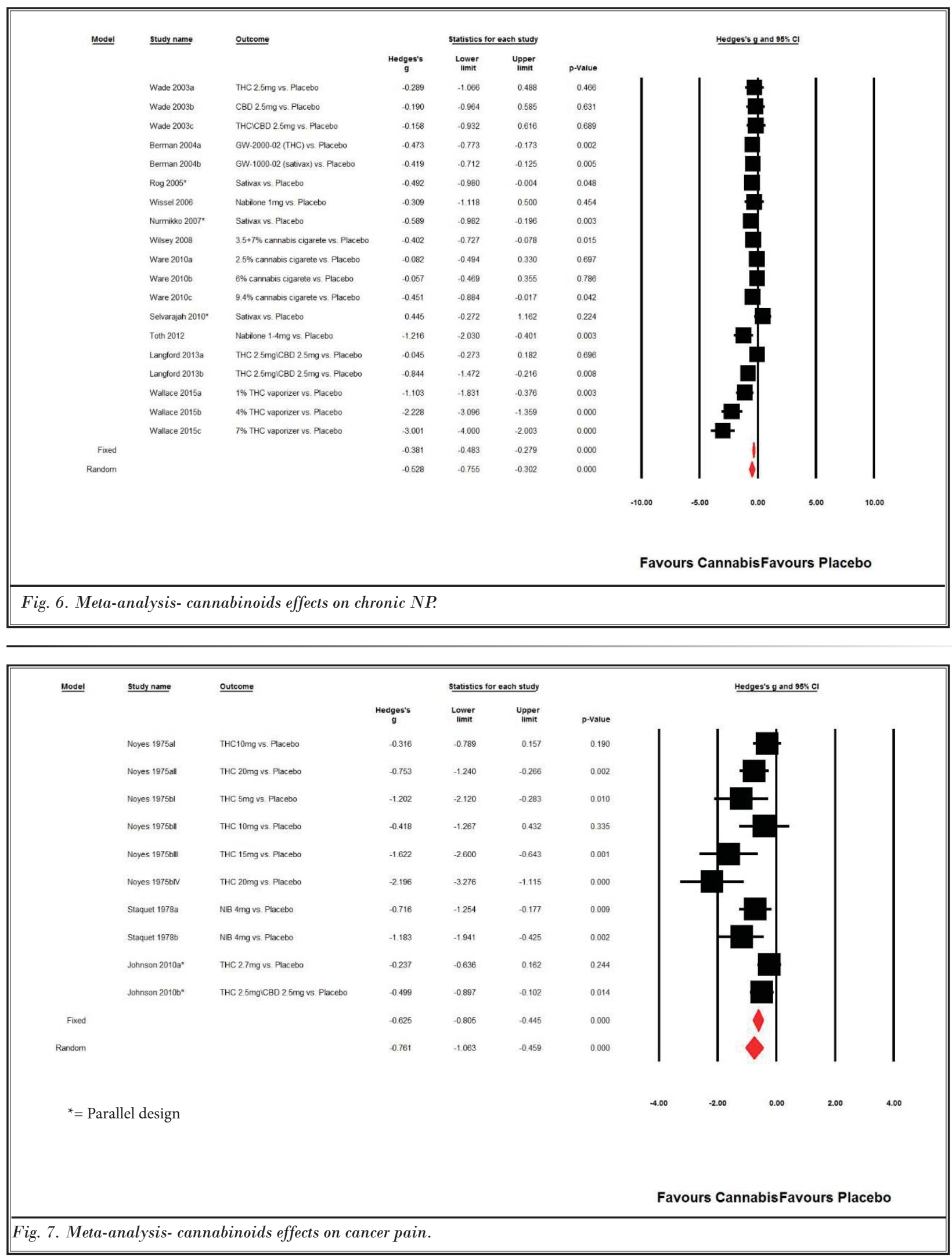


Effective of Cannabis-Based Medicines for Pain Management

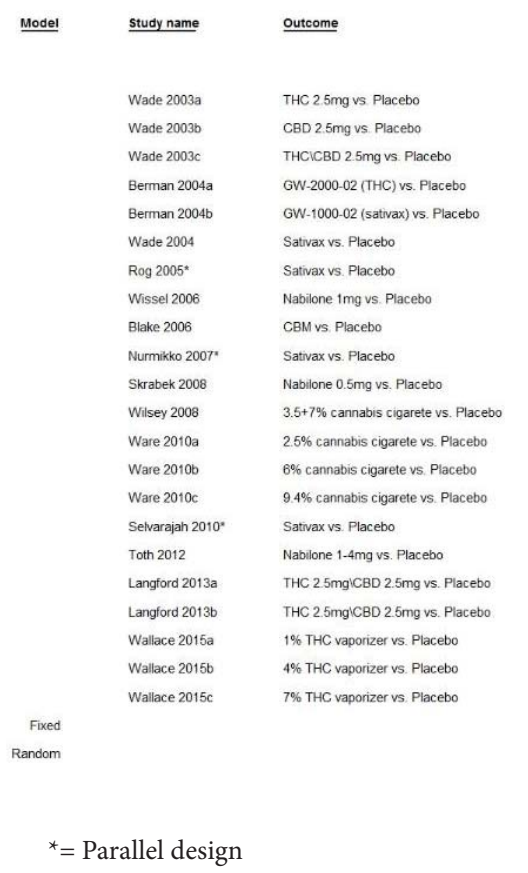

\begin{tabular}{ccc} 
Hedges's & \multicolumn{2}{c}{ statistics for each study } \\
& $\begin{array}{c}\text { Lower } \\
\text { limit }\end{array}$ & $\begin{array}{c}\text { Upper } \\
\text { limit }\end{array}$ \\
-0.289 & -1.066 & 0.488 \\
-0.190 & -0.964 & 0.585 \\
-0.158 & -0.932 & 0.616 \\
-0.473 & -0.773 & -0.173 \\
-0.419 & -0.712 & -0.125 \\
-0.303 & -0.945 & 0.340 \\
-0.492 & -0.980 & -0.004 \\
-0.309 & -1.118 & 0.500 \\
-0.629 & -1.151 & -0.107 \\
-0.589 & -0.982 & -0.196 \\
-0.837 & -1.535 & -0.138 \\
-0.402 & -0.727 & -0.078 \\
-0.082 & -0.494 & 0.330 \\
-0.057 & -0.469 & 0.355 \\
-0.451 & -0.884 & -0.017 \\
0.445 & -0.272 & 1.162 \\
-1.216 & -2.030 & -0.401 \\
-0.045 & -0.273 & 0.182 \\
-0.844 & -1.472 & -0.216 \\
-1.103 & -1.831 & -0.376 \\
-2.228 & -3.096 & -1.359 \\
-3.001 & -4.000 & -2.003 \\
-0.397 & -0.495 & -0.299 \\
-0.531 & -0.735 & -0.327
\end{tabular}

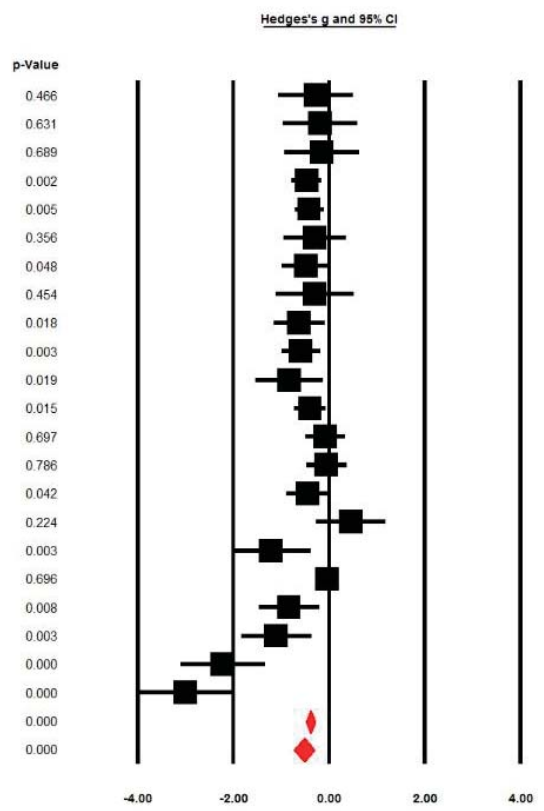

Favours CannabisFavours Placebo

Fig. 8. Meta-analysis- cannabinoids effects on chronic non-cancer pain.

current manuscript, we found that there is evidence for limited effectiveness of CBMs for pain treatment. Additionally, in most cases, the patients used additional medications (even, tough, stable dosage) for their diagnoses (Tables 1-3).

\section{Diagnoses}

The majority of the moderate to high quality studies were conducted on chronic pain, particularly on NP, due to various conditions ( $N=27)$. Few of the studies investigated cancer pain $(\mathrm{N}=7)$, however most of these studies were published few decades ago and exhibited low quality methodology. Nonetheless, several recent studies on cancer pain from 2010 to 2014 showed higher quality. Additionally, it should be mentioned that cancer pain could also have NP attributes (111).

In addition, chronic non-cancer pain was also investigated in one study of abdominal pain due to Familial Mediterranean fever (FMF), one study investigated rheumatoid arthritis, one study investigated knee osteoarthritis pain, one study investigated medicationoveruse headaches $(\mathrm{MOH})$, and one study investigated fibromyalgia. Three studies investigated postoperative pain (Tables 1-3).
Notably, although 19 of the 43 reviewed studies were not included in the meta-analyses, they showed moderate quality methodology, indicating that CBMs induced pain reduction, either by $\mathrm{N} \%$ of patients or by demonstrating significant pain reduction visually by a graph.

Furthermore, 7 studies included in this review reported significant (30-50\%) pain reduction in a substantial part of their patients. Specifically, Portenoy et al (67) showed that chronic cancer pain patients with poorly controlled opioid treatment consistently showed that low doses of nabiximols yielded significant analgesic effects. Notcutt et al (55) showed, by accumulated case reports of RCTs, that administration of oromucosal spray of THC and CBD separately, yielded $50 \%$ pain reduction in 16 of 34 chronic NP patients. Salim et al (103) showed that NP patients treated with ajulemic acid yielded $30 \%$ pain reduction in $50 \%$ of the sample compared to $20 \%$ of the sample by placebo. Ellis et al (71) showed that HIV NP patients yielded NNT of 3.5 for $30 \%$ pain reduction by cannabinoids inhalation over placebo. Zajicek et al (106) showed that MS patients reported significant clinical pain reduction by orally administered cannabis extract in $28 \%$ of the sample, compared to $17.2 \%$ in placebo. Wilsey et al (74) showed 


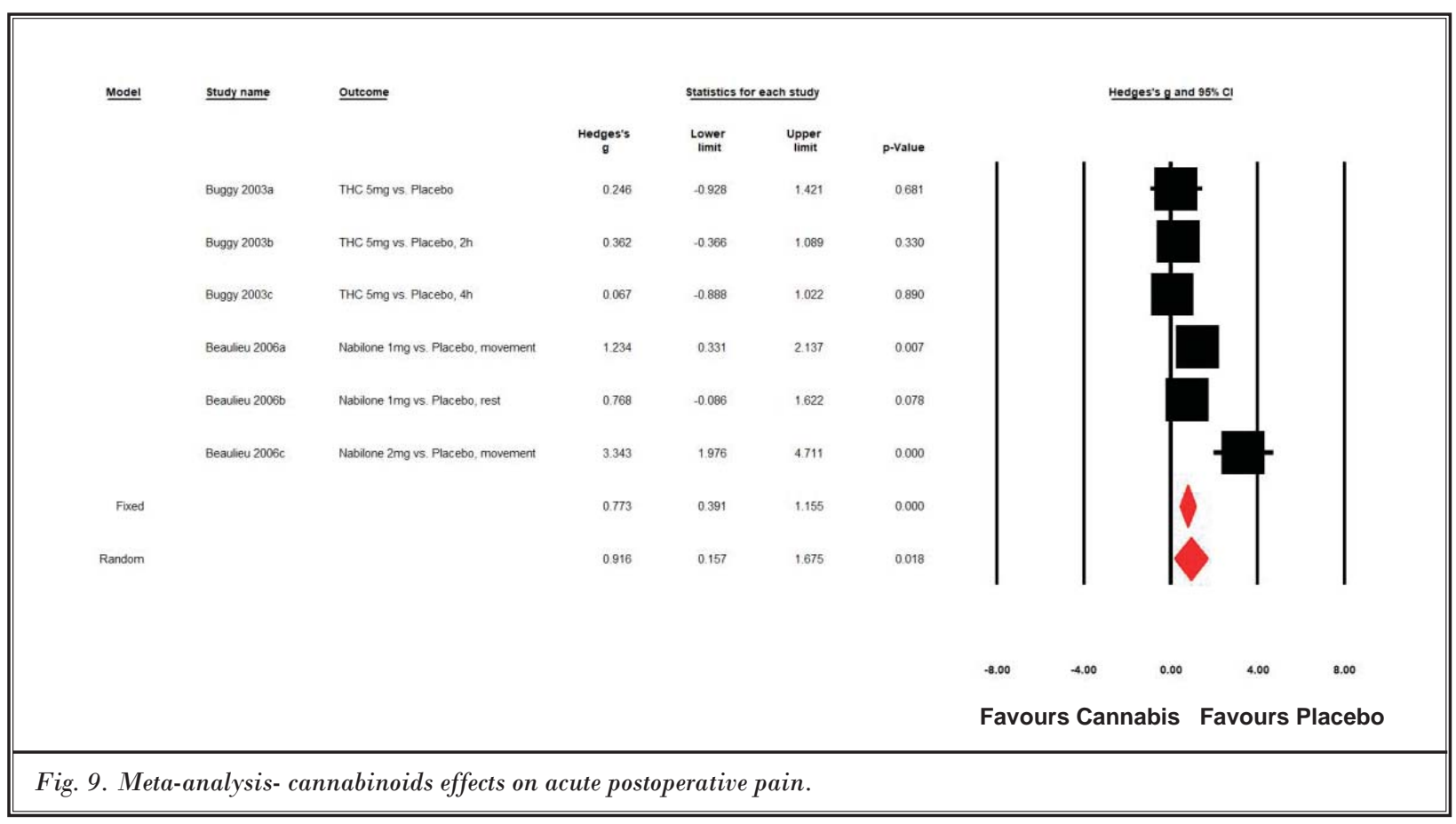

that NP patients showed NNT of 3.2 and 2.9 for $30 \%$ pain reduction by low/high THC content cannabinoids inhalation over placebo, respectively. Serpell et al (68) showed that peripheral NP patients reported significant treatment outcomes for sativex over placebo at $30 \%$ responder level (Tables 1-3).

\section{Adverse Events (AEs) Meta-Analysis}

More than half of the studies (28 of 43 RCTs) included in this review reported on AEs that were experienced by the patients in their studies (Tables 5-7). The most commonly reported AEs were for the central nervous system (CNS) and the gastro-intestinal system (GI). Other AEs were divided into groups by psychological, musculoskeletal, cardiac, vision, and hearing AEs. No separate analyses were performed for each particular AE because of the large variety of AEs; furthermore, some of the AEs were similar, but were referred to with different definitions between studies. Thus, a combined analysis was performed for each affected group. The results showed a significantly higher harm by CBMs over placebo for all the abovementioned systems, except for the musculoskeletal and cardiac systems.

Nonetheless, the participating patients in the included trials had preexisting diagnoses and in many of the trials, they used concomitant medications. For these reasons, the following AEs could not be attributed entirely to CBMs administration.

Overall, unlike the comparisons for CBMs efficacy, all of the results in the AEs analyses showed significant homogeneity. Results are demonstrated by risk ratio and $95 \%$ confidence interval form.

\section{Central Nervous System (CNS) AEs}

The combined risk ratio for all CNS-related AEs, which was reported in 26 RCTs (including apprehension, ataxia, confusion, disassociation, disconnected thought, disorientation, disturbance in attention, dizziness, dreaminess, drowsiness, falling, fatigue, feeling drunk, heavy headed, hypoesthesia, impaired balance, impaired memory, impaired psychomotor skill, incoordination, disorientation, increased awareness, lack of concentration, less alert, lethargic, lightheaded, mental clouding, numbness, slurred speech, somnolence, spasm, tiredness, and vertigo), was significantly more harmful from CBMs than by placebo, for both fixedeffect and random-effect risk ratio models 2.84 (2.16 to 3.73, $P<0.0001$ ) (Fig. 10). Homogeneity was in evidence $(I 2=0 \%, P=1.0)$ and a dispersion of Tau $2=0$, Tau $=0$.

Some of the most prevalent CNS-related AEs were dizziness and drowsiness. Dizziness was presented in 18 studies $(43,45-$ $48,52,56,62,64,65,72,89,91,96,100,104,109)$ and drowsi- 
Effective of Cannabis-Based Medicines for Pain Management

ness was presented in 10 studies $(43,46,48,52,56,65,72$ $, 91,100,104)$, both showing more harm by CBMs over placebo.

\section{Gastrointestinal (GI) AEs}

The combined risk ratio for all Gl-related AEs, which was reported in 20 RCTs (including abdominal discomfort, abdominal pain, anorexia, bad taste, constipation, decreased appetite, loss of appetite, increased appetite, diarrhea, dry mouth, dyspepsia, epigastric distress, gastric acid, glossodynia, heartburn, hypoesthesia oral, mouth dryness, mouth ulceration, mucosal erosion, nausea, oral irritation, oral pain, paresthesia oral, sore mouth, thirst, upset stomach, and vomiting), was significantly more harmful from CBMs than from placebo; for both fixed-effect and random-effect, the risk ratio models were 1.86 (1.43 to $2.43, P=0.001)$ (Fig. 11). Homogeneity was in evidence $(12=$ $0 \%, P=0.99$ ) and a dispersion of Tau2 $=0$, Tau $=0$.

Some of the most prevalent GI-related AEs were nausea and vomiting. Nausea was reported in 15 studies $(43,45-48,52,56,63-65,72,96,100,104,112)$ and vomiting was reported in 10 studies $(45,47,52,56,63-$ $65,72,104,112)$. Both were more prevalent in oromucosal and oral administration than in inhalation.

\section{Psychological AEs}

The combined risk ratio for all psychological AEs that appeared in 13 RCTs (including abnormal thinking, anxiety, confusion, craving for sweets, depressed mood, depression, disinterest in surroundings, dissociation, dysphoria, euphoria, feeling high, feeling abnormal, fidgety fingers, foggy mental state, forgetfulness, hallucinations, hyperactivity, lost in time, nervousness, nightmares, paranoia, psychological high, racing thoughts, stressful, uncooperativeness, and weird dreams) was significantly more harmful from CBMs than from placebo; for both fixed-effect and random-effect, the risk ratio models were 3.07 (1.79 to 5.26, $P<0.0001$ ) (Fig. 12). Homogeneity was in evidence (I2

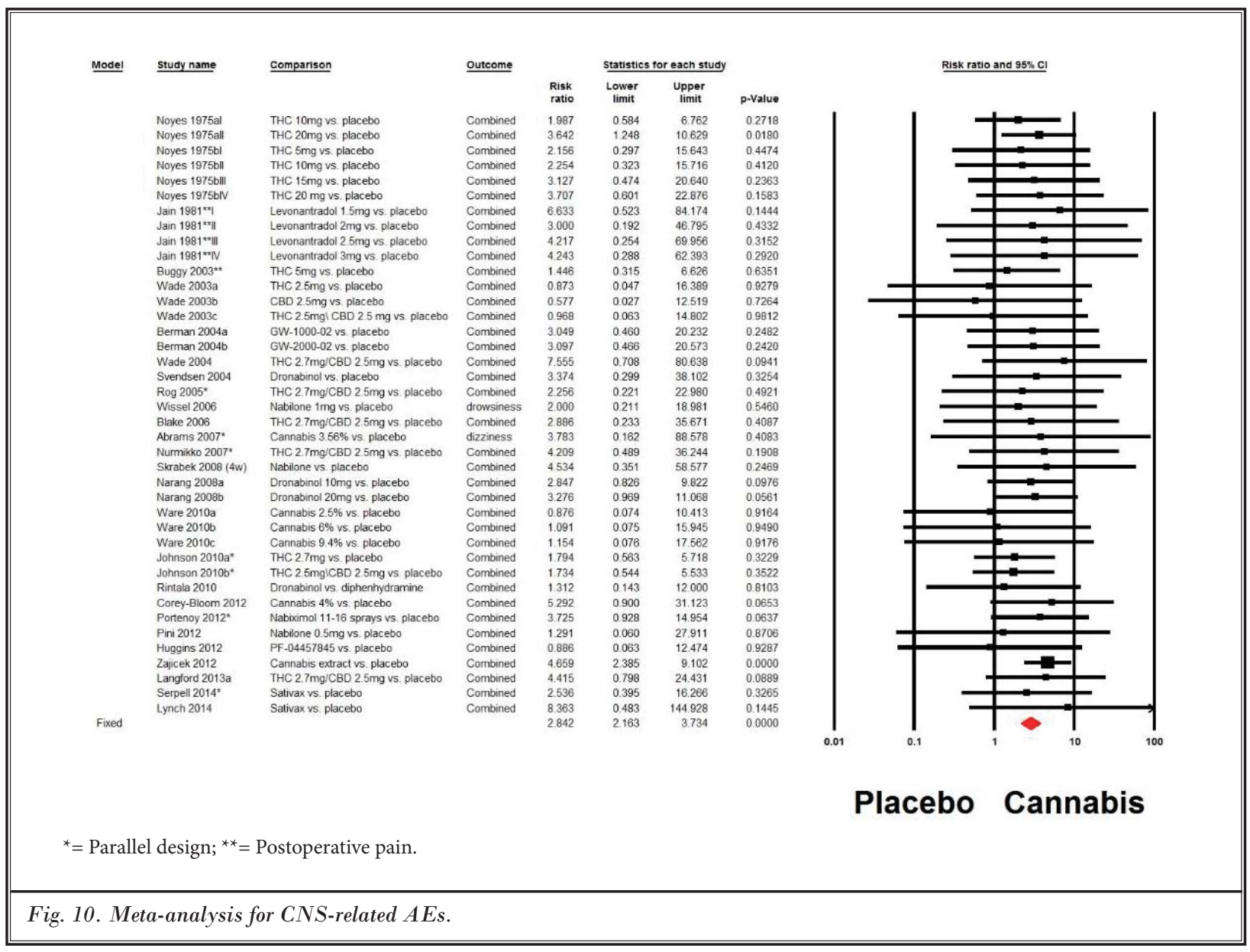


$=0 \%, P=0.99)$ and a dispersion of Tau $2=0$, Tau $=0$.

\section{Musculoskeletal AEs}

The combined risk ratio for all musculoskeletal AEs, which were reported in only 6 RCTs (including achy bones, arthritic pain, contracted jaw muscles, decreased motor skill, distortion of wrist, left back shoulder bruise, limb heaviness, limb weakness, muscle weakness, muscle twitching, musculoskeletal pain, myalgia, pain in extremity, right knee injury, and tremor), was insignificant for more harm from CBMs than from placebo; for both fixed-effect and random-effect, the risk ratio models were 1.89 (0.92 to $3.86, P=0.07$ ) (Fig. 13). Homogeneity was in evidence $(12=0 \%, P=0.93)$ and a dispersion of $\operatorname{Tau}^{2}=0$, Tau $=0$.

\section{Cardiac AEs}

The combined risk ratio for all cardiac-related AEs, which were reported in 6 RCTs (including abnormal heart rate, cardiac disorders, hypotension, orthostatic hypotension, palpitations, and tachycardia), was insig- nificant for more harm from CBMs than from placebo; for both fixed-effect and random effect, the risk ratio models were 1.49 (0.76 to 2.92, $P=0.23$ ) (Fig. 14). Homogeneity was in evidence $(12=0.0 \%, P=0.44)$ and $a$ dispersion of Tau2 $=0.0$, Tau $=0.0$. The most common cardiac-related AE was palpitations.

\section{Vision-Related AEs}

The combined risk ratio for all visual AEs, which were reported in 7 RCTs (including blurred vision, diplopia, and change in vision), was significantly more harmful from CBMs than from placebo; for fixed-effect, the risk ratio model was 3.14 (2.00 to $4.91, P<0.0001)$, and for random-effect, the risk ratio model was 2.99 (1.78 to 5.02, $P<0.0001$ ) (Fig. 15). Homogeneity was in evidence $(I 2=17.00 \%, P=0.26)$ and a dispersion of Tau2 $=0.1$, Tau $=0.41$. The most common vision-related $\mathrm{AE}$ was blurred vision.

\section{Hearing-Related AEs}

The combined risk ratio for all hearing-related AEs,

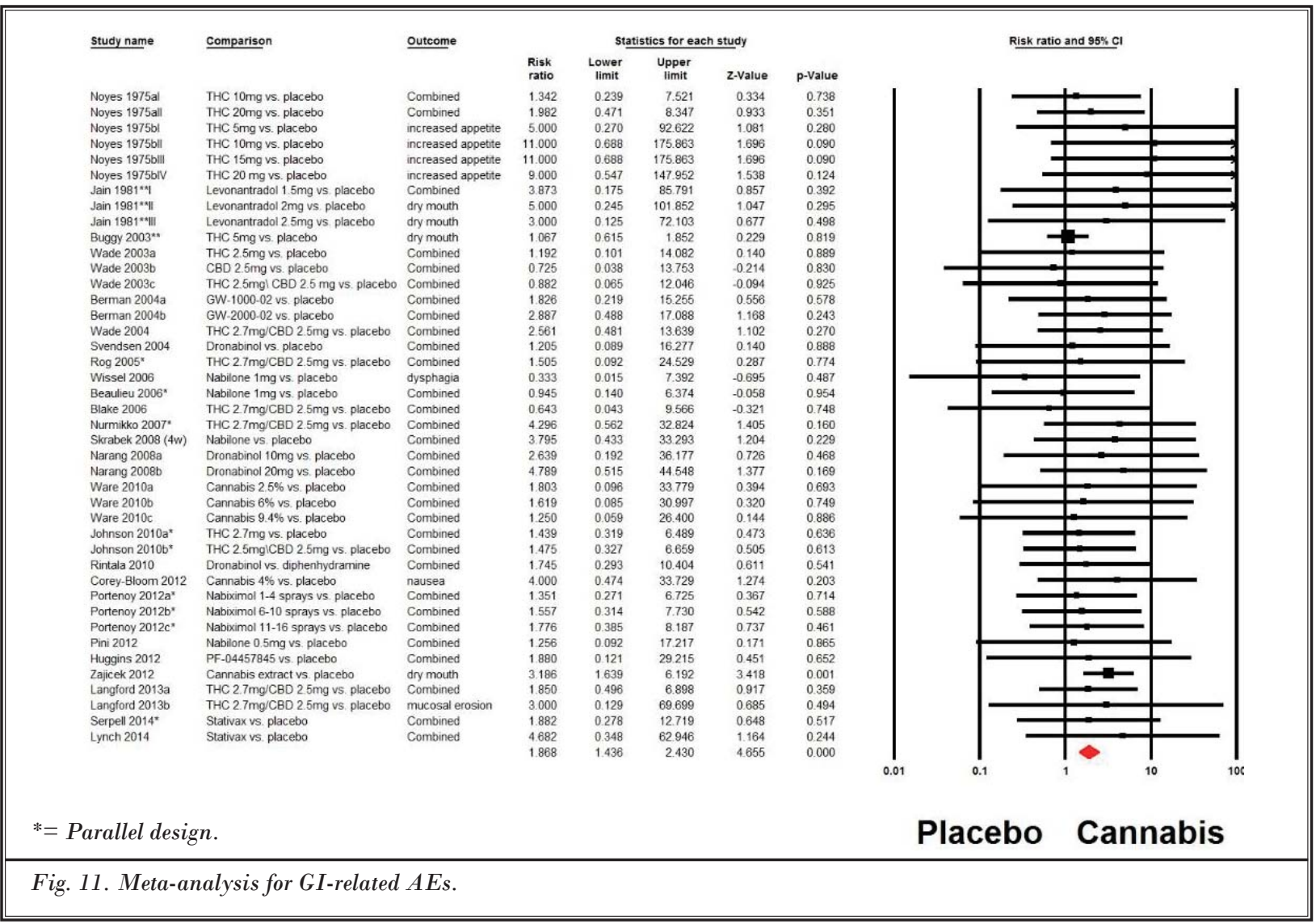


Effective of Cannabis-Based Medicines for Pain Management

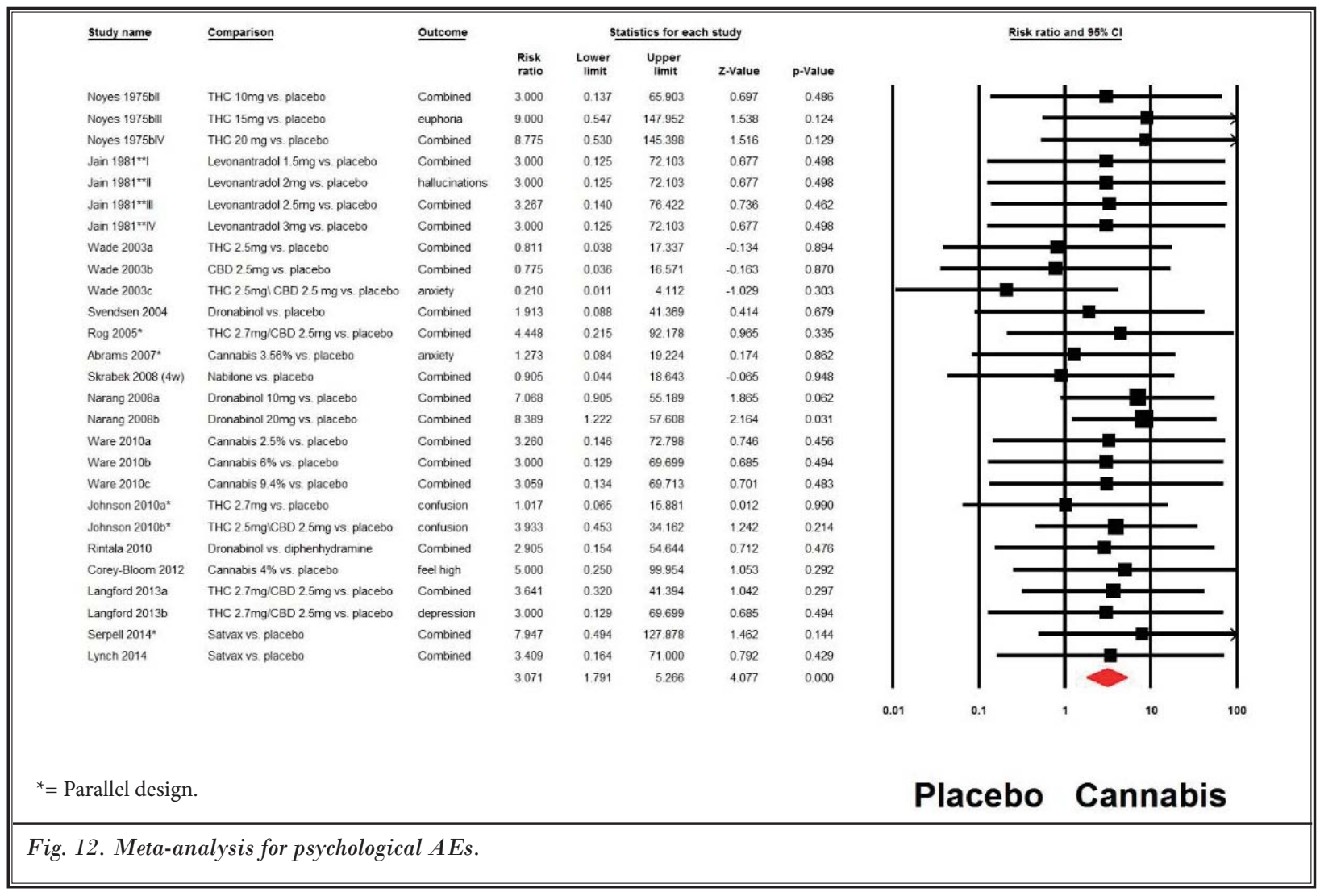

which were reported in 7 RCTs (including tinnitus, loud noise, ringing in the ears, vertigo, and ear buzzing), was significant for establishing more harm from CBMs than by placebo; for both fixed-effect and random-effect, the risk ratio models were 3.25 (1.58 to $6.67, P=0.001$ ) (Fig. 16). Homogeneity was in evidence $(I 2=0 \%, P=$ 0.81 ) and a dispersion of Tau $2=0$, Tau $=0$. Notably, the most common hearing-related AE was tinnitus, and the other terms (e.g., ringing in the ears and ear buzzing) were used in different studies to address the same AE.

\section{Miscellaneous AEs}

Many of the AEs that could not be attributed to a specific system, but were reported in the included studies are presented here: itching, sweating, headache, weakness, red eyes, drug toxicity, cough, influenzalike symptoms, application site discomfort, migraine, speech disorders, aggravation of MS symptoms, relapse of MS, hot flashes, weight decrease, fever, chills, upper respiratory tract infection, tenderness in the nose, application site burning, chest discomfort, pharyngitis, hoarseness, throat irritation, dyspnea, pain increase, nasopharyngitis, upper abdominal pain, eye irritation, facial flushing, difficulty speaking, unbalanced feeling, burning sensation, cheeks flushed, diaphoresis, heaviness, pneumonia, shortness of breath, dry eyes, raised gamma GT, hypercalcemia, rash, infections and infestations, neuralgia, pharyngolaryngeal pain, hepatobiliary disorders, hepatic enzyme increase, cognitive disorders, dysarthria, monoparesis, quadriparesis, reproductive system disorders, stress disorders, and dry skin. Due to the variability of these symptoms, they were not analyzed. Their incidence is presented in Tables 5-7. Notably, the most common AE from the above list was headache.

\section{Discussion}

\section{Summary of Evidence}

This review of 42 studies and meta-analysis of 24 RCTs is perhaps one of the most comprehensive analyses of studies that focused on the effects of cannabinoids on pain reduction and AEs to be published in recent years. This analysis found moderate to high quality of evidence for the efficacy of CBMs for treatment of chronic pain patients, especially for cancer 


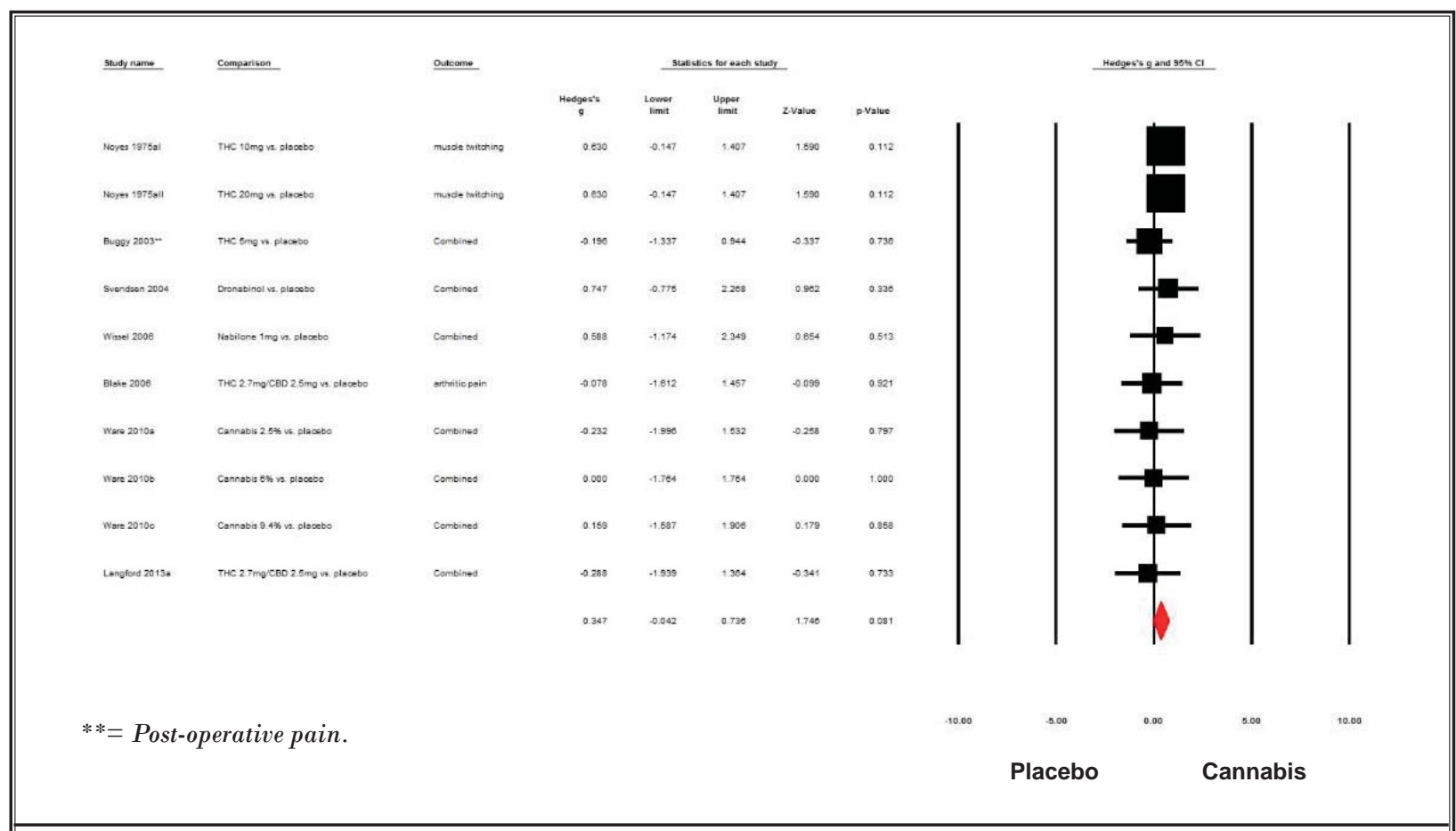

Fig. 13. Meta-analysis for musculoskeletal AEs.

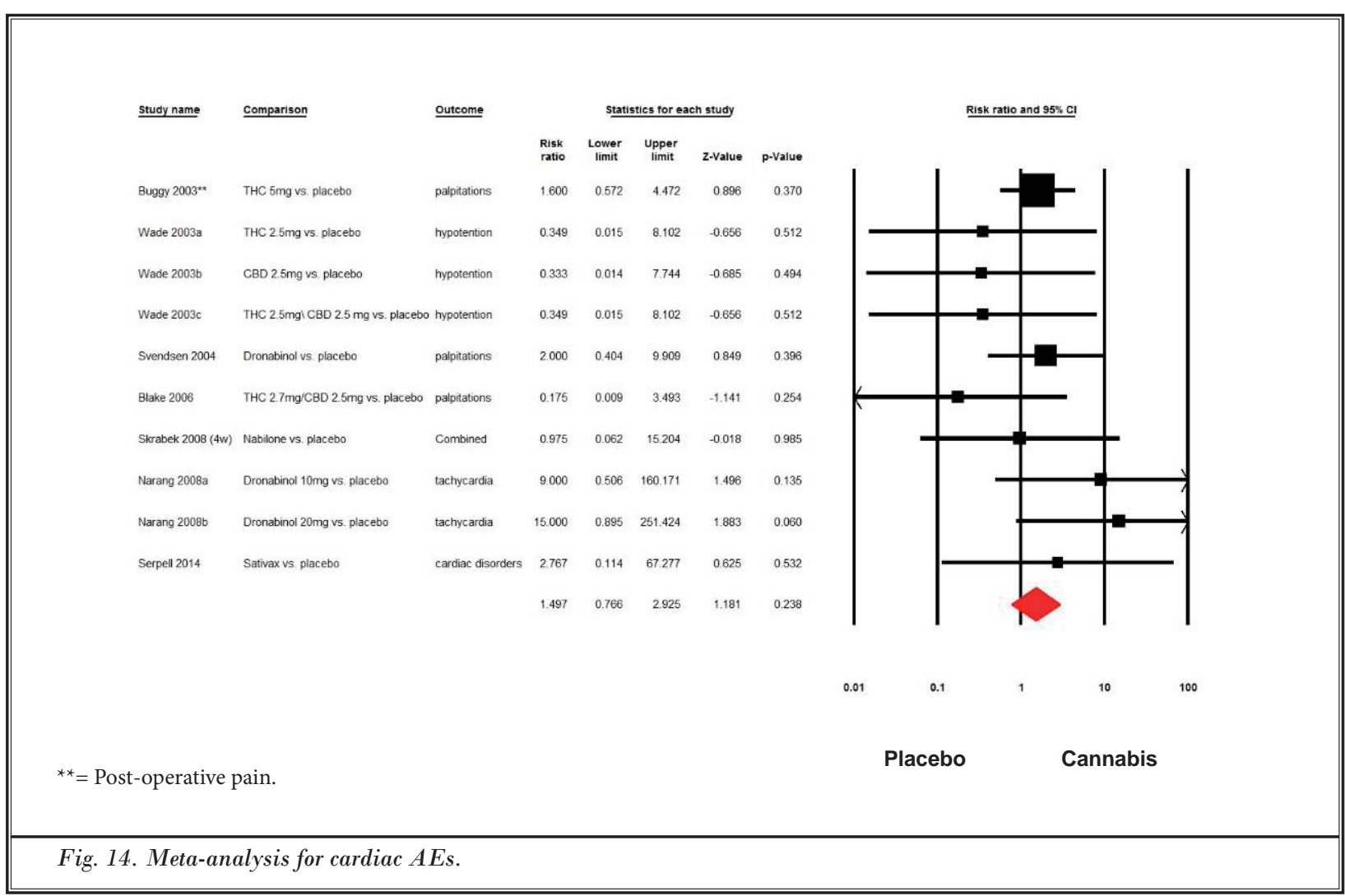




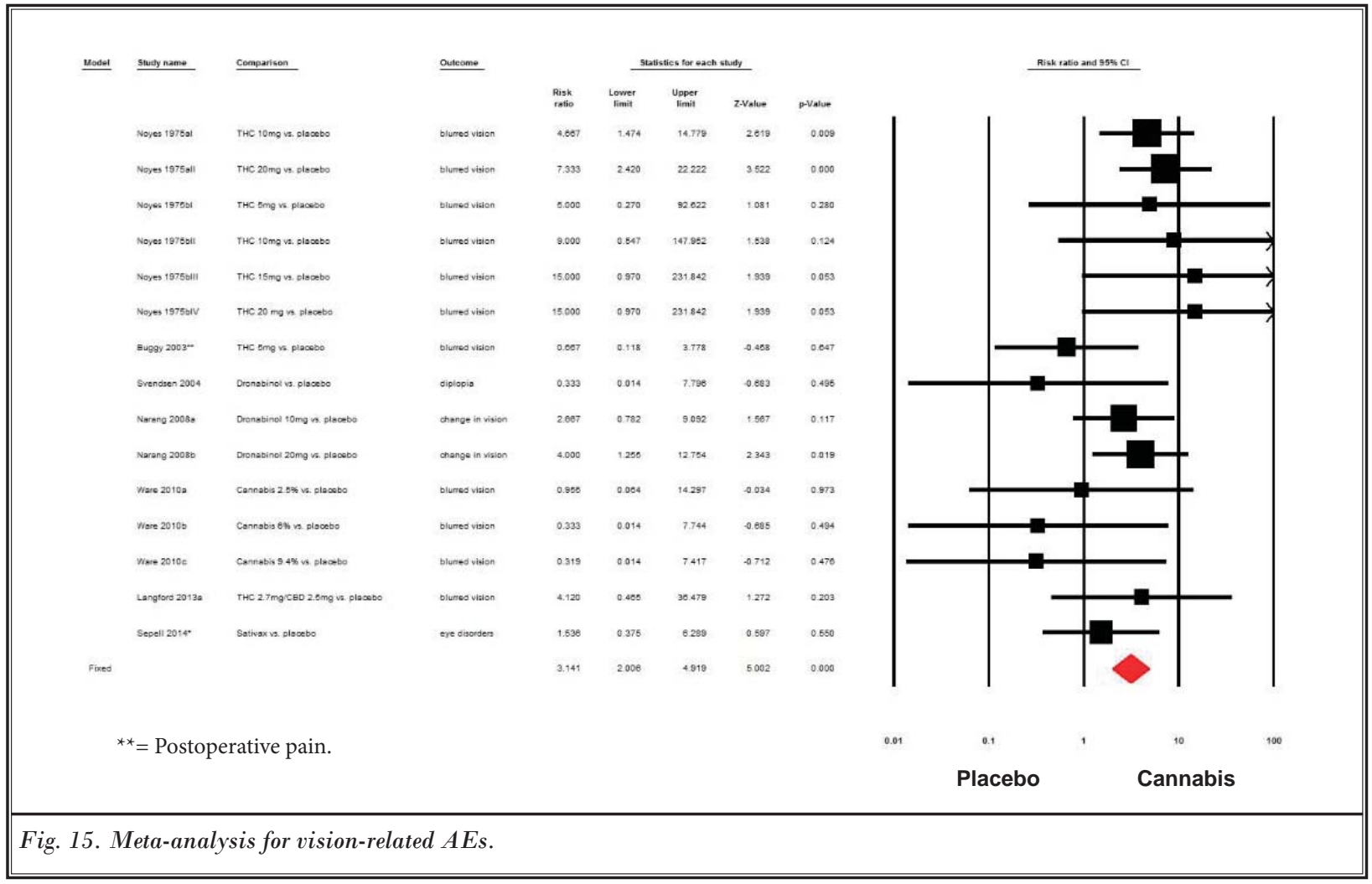

pain. However, the studies on cancer pain were scarce and mostly not from recent years, leaving chronic, noncancer pain and especially NP as the most investigated and substantiated diagnoses suitable for CBMs treatment. Conversely, postoperative pain studies showed an inverse result, where not only was there no pain reduction, in some cases, placebo was more effective than CBMs treatment.

The mode of administration in most of the studies was either oral or oromucosal, while the most rapid method of cannabinoid delivery seems to be by inhalation (13). It has been shown that when inhaling cannabinoids, plasma levels increase more rapidly and peak concentrations occur at one to 3 minutes, resulting in an analgesic effect after approximately 7 minutes (13). Furthermore, a recent international survey of 31 countries showed in approximately 1,000 patients that inhalation of cannabinoids might be the preferred route of administration, in $86.6 \%(62.9 \%$ for smoking and $23.7 \%$ for vaporizing) of the patients (12). In addition, a minority of the patients used other routes, i.e., oral and oromucosal. This preference can possibly be partially related to the slow and erratic pharmacokinetics of cannabinoids when orally administrated (112). Moreover, there was no significant difference in pharmacokinet- ics of CBMs between oral and oromucosal routes of administration (113). Additionally, although consisting of only 3 of the RCTs, the current study showed that the largest effect size for CBMs' beneficial effect on pain was found when only studies that used inhalation of cannabinoids were included in the analysis and the most promising results were shown by the most recent RCT that used a vaporizer (94). Furthermore, the results of Andreae et al's 2015 study (42), which consisted of 5 RCTs $(62,71-74)$ and analyzed $30 \%$ reduction in pain intensity as opposed to raw pain reduction, coincides with the current study's findings.

The first meta-analysis showed the same direction of results (39), but the authors did not show any use of a funnel plot analysis and, furthermore, used unpublished studies that have not made it to publication up to now, 10 years later. This renders that meta-analysis as impossible for comparison.

Surprisingly, the second meta-analysis on the analgesic value of CBMs did not use all of the available studies to perform their analysis (40), but only 7 of them (consisting of 6 publications), which were mostly recent studies (43-51). Their meta-analysis showed an overall fixed-model effect size of $-0.61(-0.84$ to -0.37$)$ for CBMs over placebo, and showed no heterogeneity $(\mathrm{I} 2=0 \%$, 


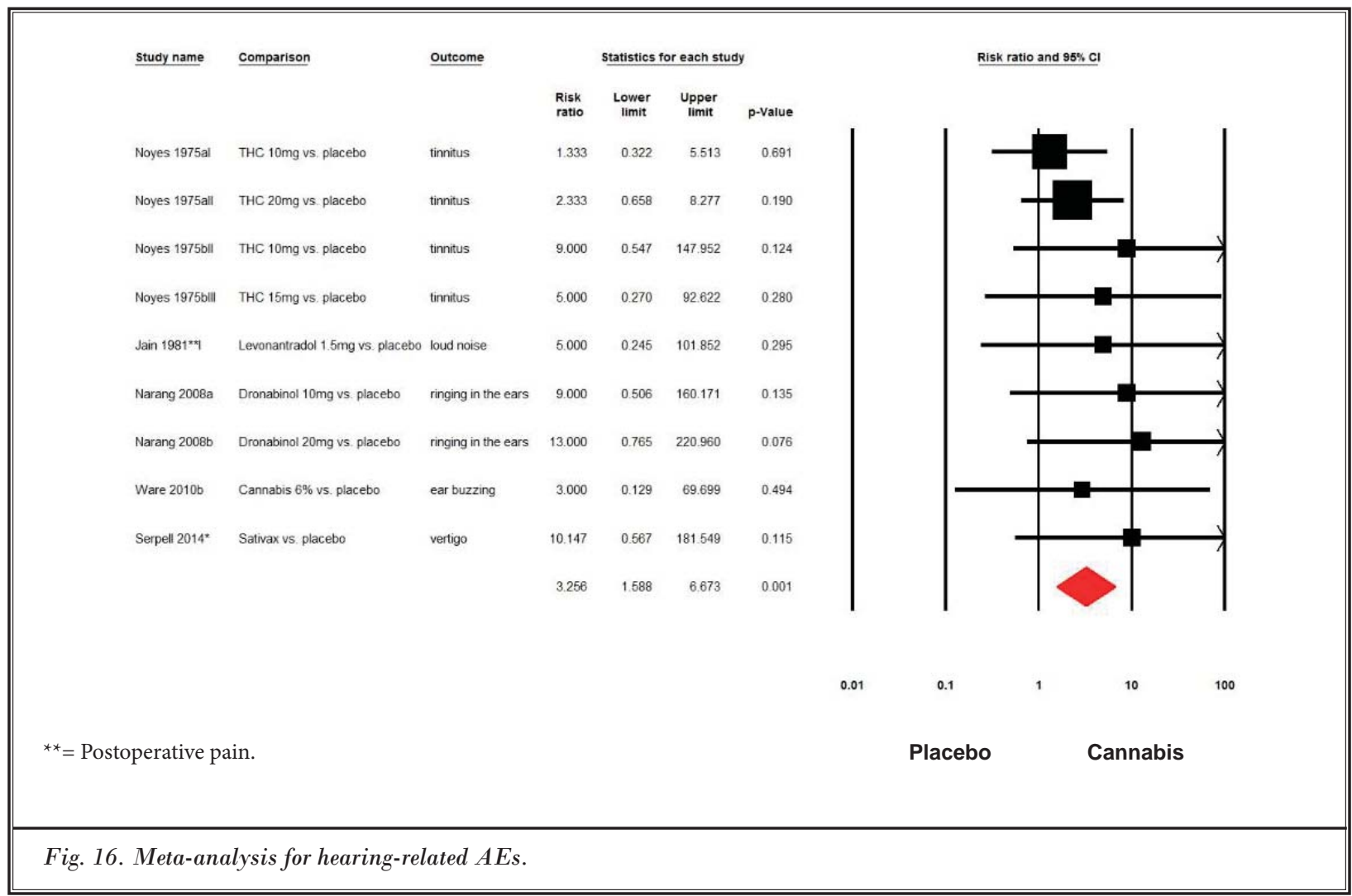

$P=0.50)$. Similarly, the current meta-analysis presented lesser values in the same direction. For comparison, the analysis, which consisted of 26 chronic pain studies (18 publications) without an active control (Fig. 4), showed a fixed-effect model of -0.45 Hedge's $g(-0.54$ to $-0.36, P$ $<0.0001)$ and a random-effect model of -0.61 Hedge's g (-0.78 to $-0.43, P<0.0001)$; a statistical heterogeneity was in evidence $(I 2=70.12 \%, P<0.0001)$. When comparing our results, no improvement was found for the efficacy of CBMs over placebo after the addition of the studies that were missing from the former meta-analysis.

The third meta-analysis of CBMs effects on pain (41) would have been the best source of comparison to the current study, but it analyzed the overall results ( 8 trials) based on the average number of patients who reported at least $30 \%$ reduction in pain, rather than the actual average reduction $(\delta)$ comparison between treatment and placebo (114). However, they also analyzed some of these trials similarly to the current study, by weighted mean differences (WMDs). Based on 6 trials, they presented a similar outcome $-0.46(95 \% \mathrm{Cl},-0.80$ to -0.11 ) to the current study's results. Their analysis of NP scale reduction in 5 trials showed a WMD of $-3.89(95 \%$ $\mathrm{Cl},-7.32$ to -0.47$)$. Additionally, a similar understanding between our study and theirs was found regarding the NP indication as the most studied indication for CBMs administration.

\section{Limitations and Methodological Considerations}

There is a substantial limitation in our study, since not all of the appropriate RCTs that were used for the review section met the inclusion criteria of the metaanalysis. If these studies could have been included, they could have altered the result of the overall effect size.

Another methodological consideration is heterogeneity. All the analyses for pain reduction efficacy showed a significant heterogeneity between the results of the studies; this could have been affected by the different cannabinoid derivates, different treatment indications between the studies and sometimes, within the studies themselves, the inclusion of both parallel and crossover designs, different trial durations, different administration routes, differences in doses, the continuation of other analgesic medication throughout 
some of the trials, the differences between the trials where the patients had prior recreational experience of cannabinoids, and the studies where the patients were naïve. The latter could have been a major limitation, since a patient who had felt the analgesic effects as well as the AEs of cannabinoids before, would have known for a high degree of certainty if they had been given the intervention/placebo arm, which in turn, could have debilitated the blinding procedure, no matter how matched/ identical the placebo was. Additionally, there was heterogeneity between the studies' washout periods. In the studies that had short washout periods, the placebo arm could have been affected by the analgesic effects of the intervention arm that was prior to it, therefore, it needs to be taken into account as a limitation. These methodological considerations should be considered in future RCTs.

In conclusion, first, based on the 3 RCTs included in this meta-analysis, CBMs were not effective for postoperative pain. Further investigation is advised. Second, there is a need for larger sample size studies of homogenous treatment indications. Third, as it can be seen in the results of the current study, inhalation is perhaps the preferred route of administration for pain relief. Future RCTs on this route of administration, with the addition of the new technologies that can produce a measured amount of inhaled THC and prevent the potential harm from noxious pyrolytic byproducts (17), may potentially turn the table and transform CBMs into a legitimate medication that can be added to the arsenal of chronic pain treatments.

\section{Clinical Implications}

Concurrently with the first meta-analysis (39), this current study needs a clinical interpretation. There is a need for a cut-point that indicates what a clinical significant reduction of pain intensity is. Farrar et al (115) stated that a reduction of 2 points on a $0-10$ numeric pain scale would be the optimal cut-off point for a clinically relevant response. Although this review consisted of some RCTs that showed a clinical response, i.e., a 2 or more point decrease $(45,56,57,60,64,92,94)$, most of the studies did not. Although, our primary analysis showed significant results favorable to CBMs over placebo. Yet, it is unclear whether our results represent any clinical significance (Fig. 2).

Notably, in most of the RCTs that were included in this review, there was a considerable incidence of AEs. However, some of these AEs can be attributed to the treated indication [i.e., arthritic pain $A E$ was found only in the study that examined rheumatoid arthritis pain (52)] or due to the rescue medication that was permitted during some studies [i.e., similar or more AEs to placebo over CBMs in postoperative pain (89)]. Furthermore, fewer overall AEs were reported when CBMs were utilized for postoperative pain, where no preexisting conditions were reported (100). Unambiguously, less GI-related AEs were reported when CBMs were administered intra muscular $(100)$ or by smoking $(62,71,72,94,96)$ compared to oral or oromucosal administration. Furthermore, more psychological AEs were reported with administration of oral dronabinol (104).

Notably, the longest list of AEs appeared when CBMs were administered by pipe smoke inhalation (72). However, these AEs appeared in a very small portion of the sample (most commonly in one of 21-22 patients). Irrefutably, the total amount of AEs that were accumulated in our meta-analysis indicates that CBMs cannot be taken lightly, and the physician should consider CBMs treatment after a complete discussion about the possible AEs that can appear. Additionally, due to the high rates of CNS-related AEs, specifically dizziness, drowsiness, and cannabinoid-related vision impairments, the patient needs to be warned not to drive a vehicle or operate heavy machinery (41).

\section{Conclusions}

The current study's results suggest that medicinal use of cannabinoids should be further investigated for chronic pain treatment, either as a single treatment, or as a combination treatment with the more conventional treatments, such as opioids and anti-NP medications, as we are not aware of the possible AEs of combination treatments with CBMs.

In comparison to other indications, CBMs have most extensively been investigated on NP (42) and evidence suggests a moderate to good treatment effect. Furthermore, NP patients should be advised that the inhalation of cannabinoids showed relatively better pain reduction effects than other routes of administration. However, the inhalation route of administration for cannabinoids for medical treatment is not followed universally (i.e., in some countries inhalation of cannabinoids is not permitted).

\section{Acknowledgments}

I would like to thank Rohtem Aviram, M.Sc and Ofrit Bar-Bachar, M.Sc for their help in English language editing. Rohtem Aviram and Ofrit Bar-Bachar have no conflict of interest or commercial association to declare. 


\section{References}

1. Cherkin DC, Sherman KJ, Balderson BH, Turner JA, Cook AJ, Stoelb B, Herman PM, Deyo RA, Hawkes RJ. Comparison of complementary and alternative medicine with conventional mind-body therapies for chronic back pain: Protocol for the Mind-body Approaches to Pain (MAP) randomized controlled trial. Trials 2014; 15:211-215.

2. Lee C, Crawford C, Buckenmaier C, Schoomaker E, Delgado R, York A. Active, self-care complementary and integrative medicine therapies for the management of chronic pain symptoms: A rapid evidence assessment of the literature. J Altern Complement Med 2014; 20:A137-A138.

3. Breen J. Transitions in the concept of chronic pain. ANS Adv Nurs Sci 2002; 24:48-59.

4. Breivik H, Collett B, Ventafridda V, Cohen R, Gallacher D. Survey of chronic pain in Europe: Prevalence, impact on daily life, and treatment. Eur] Pain 2006; 10:287-287.

5. Pain S. A potted history. Nature 2015; 525:S10-S11.

6. O'Shaughnessy W. Case of tetanus, cured by a preparation of hemp (the cannabis indica). In: O'Shaughnessy W (ed). Transactions of the Medical and Physical Society of Bengal, On the preparations of Indian hemp. Calcutta Bishops Cotton Press 1839, pp 1838-1840.

7. Mechoulam R, Gaoni Y. A total synthesis of dl- $\Delta$ 1-tetrahydrocannabinol, the active constituent of hashish.

Am Chem Soc 1965; 87:3273-3275.

8. Grinspoon L. Marijuana Reconsidered. ist ed. Harvard University Press, Cambridge, 1971.

9. Ware M, Desroches J. Pain: Clinical updates. Medical cannabis and pain. IASP 2014; 22:1-5.

10. Ware MA, Doyle CR, Woods R, Lynch ME, Clark AJ. Cannabis use for chronic non-cancer pain: Results of a prospective survey. Pain 2003; 102:211-216.

11. Hall W, Degenhardt L. Adverse health effects of non-medical cannabis use. Lancet 2009; 374:1383-1391.

12. Hazekamp A, Ware MA, Muller-Vahl KR, Abrams D, Grotenhermen F. The medicinal use of cannabis and cannabinoids-an international cross-sectional survey on administration forms. J Psychoactive Drugs 2013; 45:199-210.

13. Grotenhermen F. Pharmacokinetics and pharmacodynamics of cannabinoids. Clin Pharmacokinet 2003; 42:327-360.
14. Loflin M, Earleywine M. No smoke, no fire: What the initial literature suggests regarding vapourized cannabis and respiratory risk. Can J Respir Ther 2015; 51:7-9.

15. Eisenstein M. Medical marijuana: Showdown at the cannabis corral. Nature 2015; 525:S15-S17.

16. Mechoulam R. Cannabinoids as Therapeutics. Birkhäuser, Springer Science \& Business Media, Boston, 2005.

17. Eisenberg E, Ogintz M, Almog S. The pharmacokinetics, efficacy, safety, and ease of use of a novel portable metereddose cannabis inhaler in patients with chronic neuropathic pain: A phase ra study. J Pain Palliat Care Pharmacother 2014; 28:216-225.

18. Campbell FA, Tramèr MR, Carroll $D$, Reynolds DJM, Moore RA, McQuay HJ. Are cannabinoids an effective and safe treatment option in the management of pain? A qualitative systematic review. BM] 2001; 323:13.

19. Hazekamp A, Grotenhermen F. Review on clinical studies with cannabis and cannabinoids 2005-2009. C Cannabinoids 2010; 5:1-21.

20. Amar MB. Cannabinoids in medicine: A review of their therapeutic potential. J Ethnopharmacol 2006; 105:1-25.

21. Lynch ME, Campbell F. Cannabinoids for treatment of chronic non-cancer pain: A systematic review of randomized trials. Br J Clin Pharmacol 2011; 72:735-744.

22. Fine PG, Rosenfeld MJ. The endocannabinoid system, cannabinoids, and pain. Rambam Maimonides Med J 2013; 4:1-15.

23. Aggarwal SK. Cannabinergic pain medicine: A concise clinical primer and survey of randomized-controlled trial results. Clin J Pain 2013; 29:162-171.

24. Boychuk DG, Goddard G, Mauro G, Orellana MF. The effectiveness of cannabinoids in the management of chronic nonmalignant neuropathic pain: A systematic review. J Oral Facial Pain Headache 2015; 29:7-14.

25. Lynch ME, Ware MA. Cannabinoids for the treatment of chronic non-cancer pain: An updated systematic review of randomized controlled trials. J Neuroimmune Pharmacol 2015; 10:293-301.

26. Hill KP. Medical marijuana for treatment of chronic pain and other medical and psychiatric problems: A clinical review. JAMA 2015; 313:2474-2483.

27. Baron EP. Comprehensive review of medicinal marijuana, cannabinoids, and therapeutic implications in medicine and headache: What a long strange trip it's been.... Headache 2015; 55(6):885-916.

28. Ashton JC, Milligan ED. Cannabinoids for the treatment of neuropathic pain: Clinical evidence. Curr Opin Investig Drugs 2008; 9:65-75.

29. Fontelles MIM, García CG. Role of cannabinoids in the management of neuropathic pain. CNS Drugs 2008; 22:645-653.

30. Burns TL, Ineck JR. Cannabinoid analgesia as a potential new therapeutic option in the treatment of chronic pain. Ann Pharmacother 2006; 40:251-260.

31. Pittler $\mathrm{MH}$, Ernst E. Complementary therapies for neuropathic and neuralgic pain: Systematic review. Clin J Pain 2008; 24:731-733.

32. Davis MP. Oral nabilone capsules in the treatment of chemotherapy-induced nausea and vomiting and pain. Expert Opin Investig Drugs 2008; 17(1):85-95.

33. Jawahar R, Oh U, Yang S, Lapane KL. A systematic review of pharmacological pain management in multiple sclerosis. Drugs 2013; 73:1711-1722.

34. Phillips TJ, Cherry CL, Cox S, Marshall S), Rice AS. Pharmacological treatment of painful HIV-associated sensory neuropathy: A systematic review and metaanalysis of randomised controlled trials. PLoS One 2010; 5 :e14433.

35. Richards BL, Whittle SL, Buchbinder R. Neuromodulators for pain management in rheumatoid arthritis. Cochrane Database Syst Rev 2012; 1:CDoo8921. doi:10.1002/14651858.CDoo8921.pub2.

36. Parsai S, Herman R, Johnson S. Systematic literature review of randomized controlled trials to evaluate the efficacy of medical marijuana for analgesia. Pharmacotherapy 2014; 34:287.

37. Kung T, Hochman J, Sun Y, Bessette L, Haraoui B, Pope J, Bykerk V. Efficacy and safety of cannabinoids for pain in musculoskeletal diseases: A systematic review and meta-analysis. ] Rheumatol 2011; 38:1171-1171.

38. Canadian Agency for Drugs and Technologies in Health (CADTH). Cannabinoids for the management of neuropathic pain: review of clinical effectiveness. 2010; Ottawa, Canada. \{HOLLY: Unsure about this one

39. Iskedjian M, Bereza B, Gordon A, Piwko C, Einarson TR. Meta-analysis of cannabis based treatments for neuropathic and multiple sclerosis-related pain. Curr Med Res Opin 2006; 23:17-24.

40. Martín-Sánchez E, Furukawa TA, Tay- 
lor J, Martin JLR. Systematic review and meta-analysis of cannabis treatment for chronic pain. Pain Med 2009; 10:1353-1368.

41. Whiting PF, Wolff RF, Deshpande S, Di Nisio M, Duffy S, Hernandez AV, Keurentjes JC, Lang S, Misso K, Ryder S, Schmidlkofer S, Westwood M, Kleijnen J. Cannabinoids for medical use: A systematic review and meta-analysis. JAMA 2015; 313:2456-2473.

42. Andreae $M H$, Carter GM, Shaparin N, Suslov K, Ellis RJ, Ware MA, Abrams DI, Prasad H, Wilsey B, Indyk D, Johnson M, Sacks HS. Inhaled cannabis for chronic neuropathic pain: A meta-analysis of individual patient data. J Pain 2015; 16(12):1221-1232.

43. Berman JS, Symonds C, Birch R. Efficacy of two cannabis based medicinal extracts for relief of central neuropathic pain from brachial plexus avulsion: Results of a randomised controlled trial. Pain 2004; 112:299-306.

44. Karst M, Salim K, Burstein S, Conrad I, Hoy L, Schneider U. Analgesic effect of the synthetic cannabinoid CT-3 on chronic neuropathic pain: A randomized controlled trial. JAMA 2003; 290:1757-1762.

45. Rog DJ, Nurmikko TJ, Friede T, Young CA. Randomized, controlled trial of cannabis-based medicine in central pain in multiple sclerosis. Neurology 2005; 65:812-819.

46. Svendsen KB, Jensen TS, Bach FW. Does the cannabinoid dronabinol reduce central pain in multiple sclerosis? Randomised double blind placebo controlled crossover trial. BMJ 2004; 329:253.

47. Wade DT, Robson P, House H, Makela $\mathrm{P}$, Aram J. A preliminary controlled study to determine whether whole-plant cannabis extracts can improve intractable neurogenic symptoms. Clin Rehabil 2003; 17:21-29.

48. Wade DT, Makela P, Robson P, House $\mathrm{H}$, Bateman C. Do cannabis-based medicinal extracts have general or specific effects on symptoms in multiple sclerosis? A double-blind, randomized, placebo-controlled study on 160 patients. Mult Scler 2004; 10:434-441.

49. Bosworth T. Clinical study report: a double blind, randomised, parallel group, placebo-controlled trial of a combination of delta-9-tetrahydrocannabinol (THC) and cannabidiol (CBD) in patients with multiple sclerosis, followed by an open label assessment and study ex- tension [Study Code: GWMSoool, GW Pharma Ltd]. 2003; 1-228.

50. Dempster B. Clinical study report: a multi centre randomised, double blind, placebo controlled, parallel group comparison of the effects of cannabis based medicine standardised extracts over 4 weeks, in patients with chronic refractory pain due to multiple sclerosis or other defects of neurological function [Study Code: GWPSo105, GW Pharma Ltd]. 2003; 1-259.

51. Davidson A. Clinical study report: a double blind, randomised, parallel group placebo controlled study of cannabis based medicine extract (CBME) to validate the neuropathic pain scale (NPS) compared with a single pain severity score, in central neuropathic pain in multiple sclerosis [Study Code:GWMSo107, GW Pharma Ltd]. 2003; 1-237.

52. Blake DR, Robson P, Ho M, Jubb RW, McCabe CS. Preliminary assessment of the efficacy, tolerability and safety of a cannabis-based medicine (Sativex) in the treatment of pain caused by rheumatoid arthritis. Rheumatology (Oxford) 2006; 45:50-52.

53. Jochimsen PR, Lawton RL, VerSteeg K, Noyes R Jr. Effect of benzopyranoperidine, a delta-9-THC congener, on pain. Clin Pharmacol Ther 1978; 24:223-227.

54. Killestein J, Hoogervorst EL, Reif M, Kalkers NF, Van Loenen AC, Staats PG, Gorter RW, Uitdehaag BM, Polman CH. Safety, tolerability, and efficacy of orally administered cannabinoids in MS. Neurology 2002; 58:1404-1407.

55. Notcutt W, Price M, Miller R, Newport S, Phillips C, Simmons S, Sansom C. Initial experiences with medicinal extracts of cannabis for chronic pain: Results from 34 ' $\mathrm{N}$ of I'studies. Anaesthesia 2004; 59:440-452.

56. Noyes R Jr, Brunk SF, Baram DA, Canter A. Analgesic effect of delta-9-tetrahydrocannabinol. J Clin Pharmacol 1975; 15:139-143.

57. Noyes R Jr, Brunk SF, Avery DA, Canter AC. The analgesic properties of delta9-tetrahydrocannabinol and codeine. J Clin Pharmacol 1975; 18:84-89.

58. Pinsger $M$, Schimetta $W$, Volc D, Hiermann E, Riederer F, Pölz W. Benefits of an add-on treatment with the synthetic cannabinomimetic nabilone on patients with chronic pain--a randomized controlled trial. Wien Klin Wochenschr 2006; 118:327-335.

59. Skrabek RQ, Galimova L, Ethans K, Perry
D. Nabilone for the treatment of pain in fibromyalgia. J Pain 2008; 9:164-173.

6o. Staquet M, Gantt C, Machin D. Effect of a nitrogen analog of tetrahydrocannabinol on cancer pain. Clin Pharmacol Ther 1978; 23:397-401.

61. Wissel J, Haydn T, Müller J, Brenneis C, Berger T, Poewe W, Schelosky LD. Low dose treatment with the synthetic cannabinoid Nabilone significantly reduces spasticity-related pain. J Neurol 2006; 253:1337-1341.

62. Abrams DI, Jay CA, Shade SB, Vizoso H, Reda H, Press S, Kelly ME, Rowbotham MC, Petersen KL. Cannabis in painful HIV-associated sensory neuropathy: A randomized placebo-controlled trial. Neurology 2007; 68:515-521.

63. Johnson JR, Burnell-Nugent M, Lossignol D, Ganae-Motan ED, Potts R, Fallon MT. Multicenter, double-blind, randomized, placebo-controlled, parallel-group study of the efficacy, safety, and tolerability of THC: CBD extract and THC extract in patients with intractable cancer-related pain. J Pain Symptom Manage 2010; 39:167-179.

64. Langford R, Mares J, Novotna A, Vachova M, Novakova I, Notcutt W, Ratcliffe S. A double-blind, randomized, placebocontrolled, parallel-group study of THC/ CBD oromucosal spray in combination with the existing treatment regimen, in the relief of central neuropathic pain in patients with multiple sclerosis. J Neurol 2013; 260:984-997.

65. Nurmikko TJ, Serpell MG, Hoggart B, Toomey PJ, Morlion BJ, Haines D. Sativex successfully treats neuropathic pain characterised by allodynia: A randomised, double-blind, placebocontrolled clinical trial. Pain 2007; 133:210-220.

66. Selvarajah D, Gandhi R, Emery CJ, Tesfaye S. Randomized placebo-controlled double-blind clinical trial of cannabisbased medicinal product (Sativex) in painful diabetic neuropathy: Depression is a major confounding factor. Diabetes Care 2010; 33:128-130.

67. Portenoy RK, Ganae-Motan ED, Allende $S$, Yanagihara R, Shaiova L, Weinstein $S$, McQuade R, Wright S, Fallon MT. Nabiximols for opioid-treated cancer patients with poorly-controlled chronic pain: A randomized, placebo-controlled, graded-dose trial. J Pain 2012; 13:438-449.

68. Serpell M, Ratcliffe S, Hovorka J, Schofield M, Taylor L, Lauder H, Ehler E. A 
double-blind, randomized, placebocontrolled, parallel group study of THC/CBD spray in peripheral neuropathic pain treatment. Eur J Pain 2014; 18:999-1012.

69. GW Pharma Ltd. A double blind, randomised, placebo controlled parallel group study of sativax in the treatment of subjects with pain due to diabetic neuropathy. EU Clinical Trials Register. EU Clinical Trials Register 2004; 2014:http://clinicaltrialsregister. eu//ctr-search?query=eudract_number:2004-002530-20.

70. Whiting P, Wolff R. Medical Use of Cannabinoids-Reply. JAMA 2015; 314:1751-1752.

71. Ellis RJ, Toperoff W, Vaida F, van den Brande G, Gonzales J, Gouaux B, Bentley $\mathrm{H}$, Atkinson $\mathrm{JH}$. Smoked medicinal cannabis for neuropathic pain in HIV: A randomized, crossover clinical trial. Neuropsychopharmacology 2009; 34:672-680.

72. Ware MA, Wang T, Shapiro S, Robinson A, Ducruet T, Huynh T, Gamsa A, Bennett GJ, Collet JP. Smoked cannabis for chronic neuropathic pain: A randomized controlled trial. CMA] 2010; 182:694-701.

73. Wilsey B, Marcotte T, Tsodikov A, Millman J, Bentley $\mathrm{H}$, Gouaux B, Fishman S. A randomized, placebo-controlled, crossover trial of cannabis cigarettes in neuropathic pain. J Pain 2008; 9:506-521.

74. Wilsey B, Marcotte $T$, Deutsch R, Gouaux B, Sakai S, Donaghe H. Lowdose vaporized cannabis significantly improves neuropathic pain. J Pain 2013; 14:136-148.

75. Dworkin RH, O'Connor AB, Backonja $M$, Farrar JT, Finnerup NB, Jensen TS, Kalso EA, Loeser JD, Miaskowski C, Nurmikko TJ, Portenoy RK, Rice AS, Stacey BR, Treede RD, Turk DC, Wallace MS. Pharmacologic management of neuropathic pain: Evidence-based recommendations. Pain 2007; 132:237-251.

76. Farrar JT, Troxel AB, Stott C, Duncombe $P$, Jensen MP. Validity, reliability, and clinical importance of change in a o-10 numeric rating scale measure of spasticity: A post hoc analysis of a randomized, double-blind, placebo-controlled trial. Clin Ther 2008; 30:974-985.

77. Moore RA, Wiffen PJ, Derry S, Toelle T, Rice AS. Gabapentin for chronic neuropathic pain and fibromyalgia in adults. Cochrane Database Syst Rev 2014; 4:CDoo7938. doi: 10.1002/14651858. CDoo7938.pub3.

78. Wiffen PJ, Derry S, Moore RA, Aldington
D, Cole P, Rice AS, Lunn MP, Hamunen K, Haanpaa M, Kalso EA. Antiepileptic drugs for neuropathic pain and fibromyalgiaDan overview of Cochrane reviews. Cochrane Database Syst Rev 2013. 11:CDo10567. doi: $10.1002 / 14651858$. CD10567.pub2.

79. Birse F, Derry S, Moore RA. Phenytoin for neuropathic pain and fibromyalgia in adults. Cochrane Database Syst Rev 2012; 5:CDoo9485. doi: 10.1002/14651858. CDoog485.pub2.

8o. Chaparro LE, Wiffen PJ, Moore RA, Gilron I. Combination pharmacotherapy for the treatment of neuropathic pain in adults. Cochrane Database Syst Rev 2012; 7:CDoo8943. doi: 10.1002/14651858. CDoo8943.pub2.

81. Corrigan R, Derry S, Wiffen PJ, Moore RA. Clonazepam for neuropathic pain and fibromyalgia in adults. Cochrane Database Syst Rev 2012; 5:CDoo9486. doi: 10.1002/14651858.CDoo9486.pub2.

82. Deshpande A, Mailis-Gagnon A, Zoheiry N, Lakha SF. Efficacy and adverse effects of medical marijuana for chronic noncancer pain: Systematic review of randomized controlled trials. Can Fam Physician 2015; 61:372-81.

83. Moher D, Liberati A, Tetzlaff J, Altman DG; PRISMA Group. Preferred reporting items for systematic reviews and meta-analyses: The PRISMA statement. Ann Intern Med 2009; 151:264-269.

84. Jadad AR, Moore RA, Carroll D, Jenkinson C, Reynolds DJM, Gavaghan DJ, McQuay HJ. Assessing the quality of reports of randomized clinical trials: Is blinding necessary? Control Clin Trials 1996; 17:1-12.

85. Hartrick CT, Kovan JP, Shapiro S. The numeric rating scale for clinical pain measurement: A ratio measure? Pain Pract 2003; 3:310-316.

86. Jensen MP, Karoly P, O'Riordan EF, Bland F Jr, Burns RS. The subjective experience of acute pain. An assessment of the utility of 10 indices. Clin J Pain 1989; 5:153-159.

87. Huskisson EC. Measurement of pain. J Rheumatol 1982; 9:768-769.

88. Melzack R. The short-form McGill Pain Questionnaire. Pain 1987; 30:191-197.

89. Buggy DJ, Toogood L, Maric S, Sharpe P, Lambert DG, Rowbotham DJ. Lack of analgesic efficacy of oral delta-9-tetrahydrocannabinol in postoperative pain. Pain 2003; 106:169-172.

90. Frank B, Serpell MG, Hughes J, Matthews JN, Kapur D. Comparison of an- algesic effects and patient tolerability of nabilone and dihydrocodeine for chronic neuropathic pain: Randomised, crossover, double blind study. BMJ 2008; 336:199-201.

91. Rintala DH, Fiess RN, Tan G, Holmes SA, Bruel BM. Effect of dronabinol on central neuropathic pain after spinal cord injury: A pilot study. Am J Phys Med Rehabil 2010; 89:840-848.

92. Toth C, Mawani S, Brady S, Chan C, Liu C, Mehina E, Garven A, Bestard J, Korngut L. An enriched-enrolment, randomized withdrawal, flexible-dose, double-blind, placebo-controlled, parallel assignment efficacy study of nabilone as adjuvant in the treatment of diabetic peripheral neuropathic pain. Pain 2012; 153:2073-2082.

93. Beaulieu P. Effects of nabilone, a synthetic cannabinoid, on postoperative pain. Can J Anaesth 2006; 53:769-775.

94. Wallace MS, Marcotte TD, Umlauf A, Gouaux B, Atkinson JH. Efficacy of inhaled cannabis on painful diabetic neuropathy. J Pain 2015; 16(7):616-627.

95. Pini LA, Guerzoni S, Cainazzo MM Ferrari A, Sarchielli P, Tiraferri I, Ciccarese $M$, Zappaterra $M$. Nabilone for the treatment of medication overuse headache: Results of a preliminary doubleblind, active-controlled, randomized trial. J Headache Pain 2012; 13:677-684.

96. Corey-Bloom J, Wolfson T, Gamst A, Jin S, Marcotte TD, Bentley H, Gouaux B. Smoked cannabis for spasticity in multiple sclerosis: A randomized, placebo-controlled trial. CMAJ 2012; 184:1143-1150.

97. Borenstein $M$, Hedges L, Higgins J, Rothstein $\mathrm{H}$. Comprehensive metaanalysis version 2. Englewood N]: Biostat 2005; 104. $\{$ H L LY: Unsure about this one

98. Higgins JP, Thompson SG, Deeks JJ, Altman DG. Measuring inconsistency in meta-analyses. BM] 2003; 327:557-560.

99. Seeling $W$, Kneer L, Büchele $B$, Gschwend JE, Maier L, Nett C, Simmet T, Steffen P, Schneider M, Rockemann M. [Delta(9)-tetrahydrocannabinol and the opioid receptor agonist piritramide do not act synergistically in postoperative pain]. Anaesthesist 2006; 55:391-400.

100. Jain AK, Ryan JR, McMahon FG, Smith G. Evaluation of intramuscular levonantradol and placebo in acute postoperative pain. J Clin Pharmacol 1981; 21:320S-326S.

101. Maurer M, Henn V, Dittrich A, Hofmann 
A. Delta-9-tetrahydrocannabinol shows antispastic and analgesic effects in a single case double-blind trial. Eur Arch Psychiatry Clin Neurosci 1990; 240:1-4.

102. Holdcroft A, Smith M, Jacklin A, Hodgson $H$, Smith B, Newton M, Evans F. Pain relief with oral cannabinoids in familial Mediterranean fever. Anaesthesia 1997; 52:483-486.

103. Salim K, Schneider U, Burstein S, Hoy L, Karst M. Pain measurements and side effect profile of the novel cannabinoid ajulemic acid. Neuropharmacology 2005; 48:1164-1171.

104. Narang S, Gibson D, Wasan AD, Ross EL, Michna E, Nedeljkovic SS, Jamison RN. Efficacy of dronabinol as an adjuvant treatment for chronic pain patients on opioid therapy. J Pain 2008; 9:254-264.

105. Huggins JP, Smart TS, Langman S, Taylor L, Young T. An efficient randomised, placebo-controlled clinical trial with the irreversible fatty acid amide hydrolase-1 inhibitor PF-04457845, which modulates endocannabinoids but fails to induce effective analgesia in patients with pain due to osteoarthritis of the knee. Pain 2012; 153:1837-1846.

106. Zajicek JP, Hobart JC, Slade A, Barnes D, Mattison PG; MUSEC Research Group. Multiple sclerosis and extract of cannabis: Results of the MUSEC trial. J Neurol Neurosurg Psychiatry 2012; 83:1125-1132.
107. Lynch ME, Cesar-Rittenberg P, Hohmann AG. A double-blind, placebo-controlled, crossover pilot trial with extension using an oral mucosal cannabinoid extract for treatment of chemotherapyinduced neuropathic pain. J Pain Symptom Manage 2014; 47:166-173.

108. Turcotte D, Doupe M, Torabi M, Gomori A, Ethans K, Esfahani F, Galloway $\mathrm{K}$, Namaka M. Nabilone as an adjunctive to gabapentin for multiple sclerosis-induced neuropathic pain: A randomized controlled trial. Pain Med 2015; 16:149-159.

109. Johnson J, Potts R. Cannabis-based medicines in the treatment of cancer pain: A randomised, double-blind, parallel group, placebo controlled, comparative study of the efficacy, safety and tolerability of Sativex and Tetranabinex in patients with cancer-related pain. British Pain Society. Edinburgh, Scotland 2005:8-11.

110. Higgins T, Green S. Cochrane Handbook for Systematic Reviews of Interventions 4.2.6 [updated September 2006]. John Wiley \& Sons, Ltd., Chichester, 2006.

111. Boland EG, Mulvey MR, Bennett MI. Classification of neuropathic pain in cancer patients. Curr Opin Support Palliat Care 2015; 9:112-115.

112. Ohlsson A, Lindgren J, Wahlen A,
Agurell S, Hollister LE, Gillespie HK. Plasma delta-9-tetrahydrocannabino concentrations and clinical effects after oral and intravenous administration and smoking. Clin Pharmacol Ther 1980; 28:409-416.

113. Karschner EL, Darwin WD, Goodwin RS, Wright S, Huestis MA. Plasma cannabinoid pharmacokinetics following controlled oral deltag-tetrahydrocannabinol and oromucosal cannabis extract administration. Clin Chem 2011; 57:66-75.

114. Dworkin RH, Turk DC, Wyrwich KW, Beaton D, Cleeland CS, Farrar JT, Haythornthwaite JA, Jensen MP, Kerns RD, Ader DN, Brandenburg N, Burke LB, Cella D, Chandler J, Cowan P, Dimitrova R, Dionne R, Hertz S, Jadad AR, Katz NP, Kehlet $\mathrm{H}$, Kramer LD, Manning DC, McCormick C, McDermott MP, McQuay HJ, Patel S, Porter L, Quessy S, Rappaport BA, Raushkolb C, Revicki DA, Rothman M, Schmader KE, Stacey BR, Stauffer JW, von Stein T, White RE, Witter J, Zavisic $S$. Interpreting the clinical importance of treatment outcomes in chronic pain clinical trials: IMMPACT recommendations. J Pain 2008; 9:105-121.

115. Farrar JT, Portenoy RK, Berlin JA, Kinman JL, Strom BL. Defining the clinically important difference in pain outcome measures. Pain 2000; 88:287-294. 